\title{
DEVELOPMENT OF AN INTEGRATED INFORMATION MODEL FOR COMPUTER INTEGRATED MANUFACTURING
}

\author{
A Thesis Presented to \\ The Faculty of the \\ Fritz J. and Dolores H. Russ \\ College of Engineering and Technology \\ Ohio University
}

\begin{abstract}
In Partial Fulfillment
of the Requirement for the Degree
\end{abstract}

Master of Science

by

Pascal Dreer

November, 1995 


\title{
Acknowledgements
}

\author{
Du bist Künstler, ich bin Denker. \\ Narziss und Goldmund, Hermann Hesse
}

I would like to thank everybody at Ohio University who has actively helped me during my study towards this Master degree. The acquaintance of interesting and friendly people in Athens made the time at Ohio University definitely a great experience.

Finally, I wish to thank my family and friends back at home in Switzerland for their support.

Athens, Ohio, USA August 18, 1995 


\section{Table of Contents}

Acknowledgements $\ldots \ldots \ldots \ldots \ldots \ldots \ldots \ldots \ldots \ldots \ldots \ldots \ldots \ldots \ldots \ldots$ ii

Table of Contents $\ldots \ldots \ldots \ldots \ldots \ldots \ldots \ldots \ldots \ldots \ldots \ldots \ldots \ldots \ldots \ldots$ iv

List of Figures $\ldots \ldots \ldots \ldots \ldots \ldots \ldots \ldots \ldots \ldots \ldots \ldots \ldots \ldots \ldots \ldots \ldots$

List of Tables $\ldots \ldots \ldots \ldots \ldots \ldots \ldots \ldots \ldots \ldots \ldots \ldots \ldots \ldots \ldots \ldots \ldots$ viii

Introduction $\ldots \ldots \ldots \ldots \ldots \ldots \ldots \ldots \ldots \ldots \ldots \ldots \ldots \ldots \ldots \ldots \ldots$

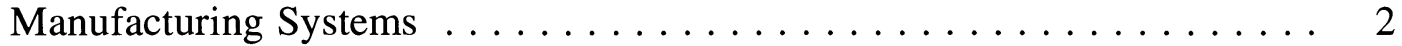

Computer Integrated Manufacturing $\ldots \ldots \ldots \ldots \ldots \ldots \ldots$

Statement of the Problem ...................... 9

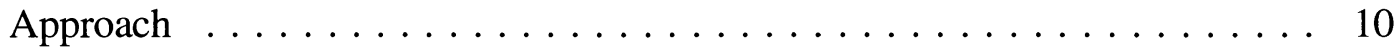

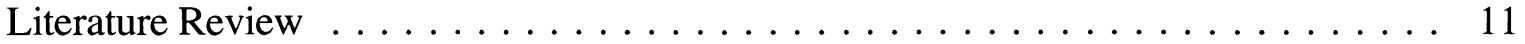

Existing CIM Models . . . . . . . . . . . . . . . . . . . . . 12

IBM CIM Architecture $\ldots \ldots \ldots \ldots \ldots \ldots \ldots \ldots \ldots \ldots$

NIST-AMRF Hierarchical Model $\ldots \ldots \ldots \ldots \ldots \ldots \ldots \ldots$

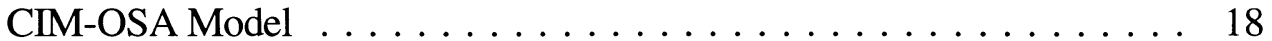

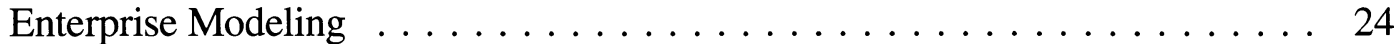

Elements of Enterprise Modeling $\ldots \ldots \ldots \ldots \ldots \ldots 25$

Databases ............................ 30

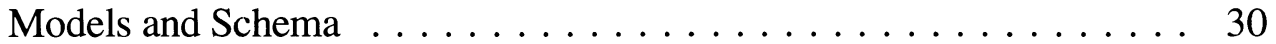

Information Modeling $\ldots \ldots \ldots \ldots \ldots \ldots \ldots \ldots \ldots \ldots \ldots$

Database Design $\ldots \ldots \ldots \ldots \ldots \ldots \ldots \ldots \ldots \ldots \ldots$

Manufacturing Databases $\ldots \ldots \ldots \ldots \ldots \ldots \ldots \ldots$

Database Integration . . . . . . . . . . . . . . . . . 37

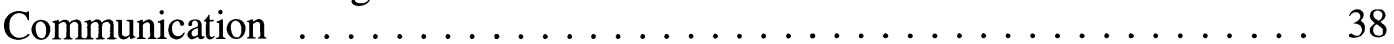

Communication Types $\ldots \ldots \ldots \ldots \ldots \ldots \ldots \ldots \ldots$

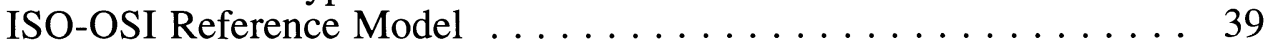

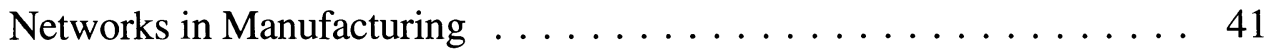

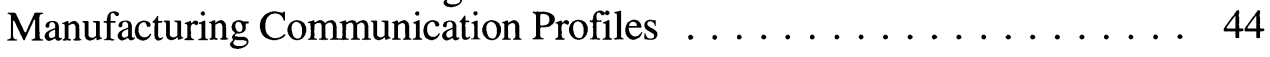

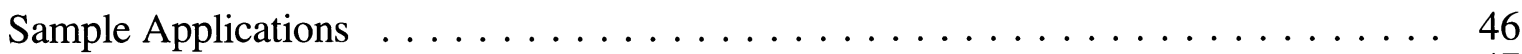

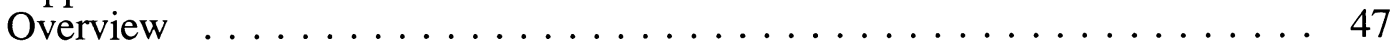

Facility Layout Tool $\ldots \ldots \ldots \ldots \ldots \ldots \ldots \ldots$

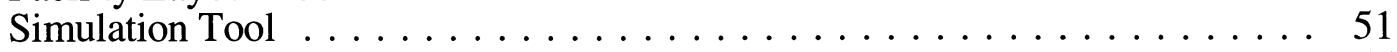

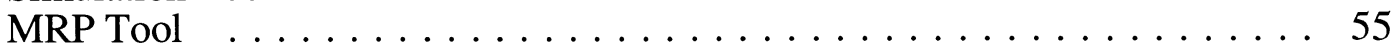

Integration Concept $\ldots \ldots \ldots \ldots \ldots \ldots \ldots \ldots \ldots \ldots \ldots \ldots \ldots \ldots \ldots \ldots \ldots$

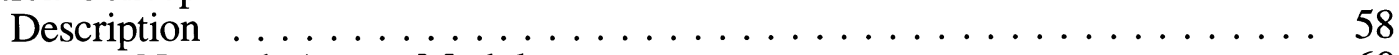

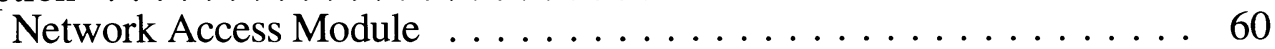

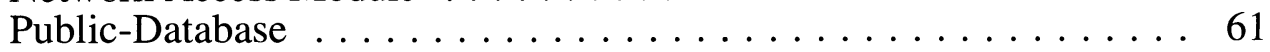

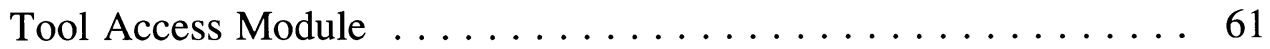

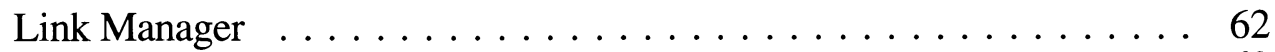

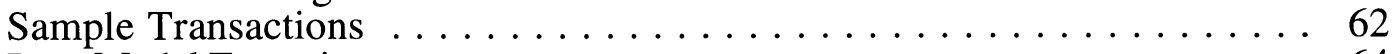

Data Model Extension . . . . . . . . . . . . . . . . . . . 64 


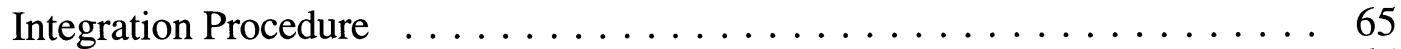

Framework Implementation $\ldots \ldots \ldots \ldots \ldots \ldots \ldots \ldots \ldots$

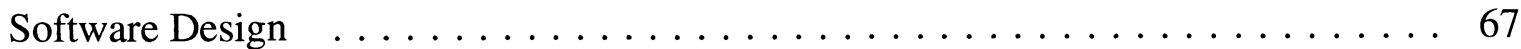

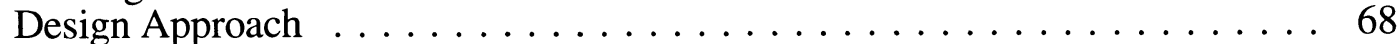

Modules ............................ 72

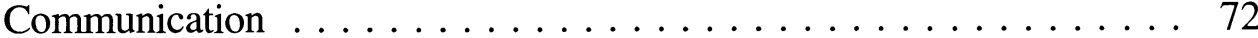

Network Access Interface $\ldots \ldots \ldots \ldots \ldots \ldots$. . . . . . . 73

IX Communication Protocol $\ldots \ldots \ldots \ldots \ldots \ldots$

Internal Public-Database $\ldots \ldots \ldots \ldots \ldots \ldots \ldots \ldots$. . . . . . . 81

Database Access Interface $\ldots \ldots \ldots \ldots \ldots$. . . . . . . . . 82

Tool Access $\ldots \ldots \ldots \ldots \ldots \ldots \ldots \ldots \ldots \ldots \ldots \ldots$

Tool Access Interface $\ldots \ldots \ldots \ldots \ldots \ldots \ldots$. . . . . . 85

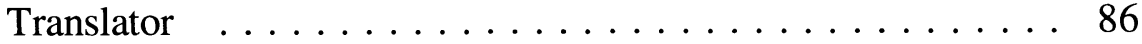

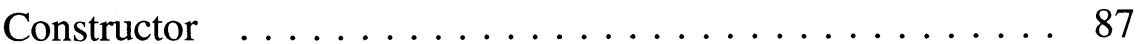

Link Manager $\ldots \ldots \ldots \ldots \ldots \ldots \ldots \ldots \ldots \ldots \ldots$

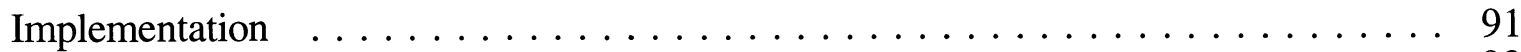

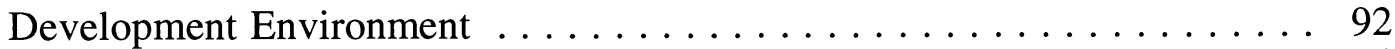

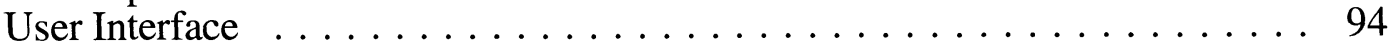

Network Access $\ldots \ldots \ldots \ldots \ldots \ldots \ldots \ldots \ldots \ldots$

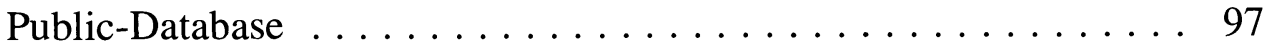

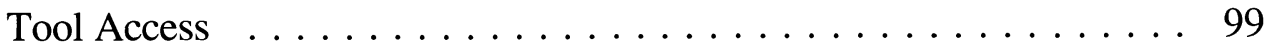

System Requirements . . . . . . . . . . . . . . . . . . . . . 100

Sample Integration $\ldots \ldots \ldots \ldots \ldots \ldots \ldots \ldots \ldots \ldots \ldots \ldots \ldots \ldots \ldots$

Sample Case Study . . . . . . . . . . . . . . . . . . . . 102

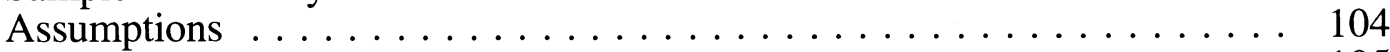

Tool Configuration ........................ 105

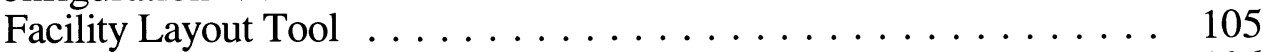

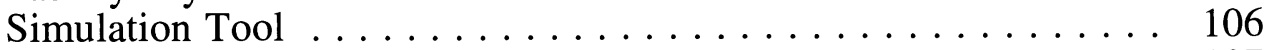

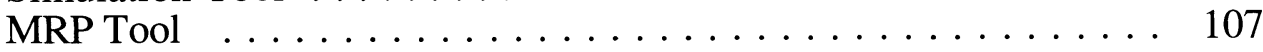

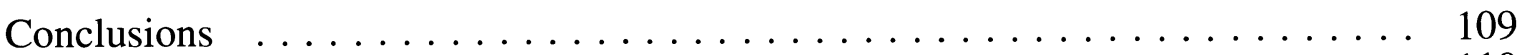

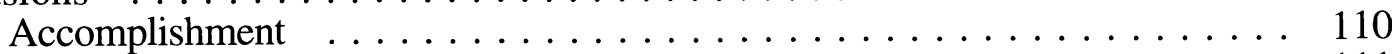

Limitations and Problems $\ldots \ldots \ldots \ldots \ldots \ldots \ldots \ldots \ldots \ldots \ldots$

Further Research . . . . . . . . . . . . . . . . . . . . . . 113

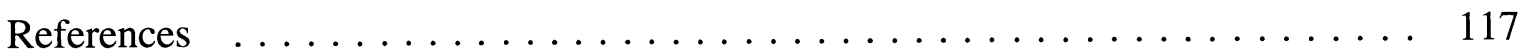

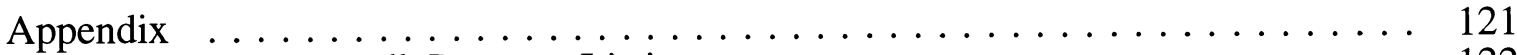

A IX HyperTalk Program Listings $\ldots \ldots \ldots \ldots \ldots \ldots \ldots \ldots \ldots \ldots, 122$

B IX External Command Program Listings $\ldots \ldots \ldots \ldots \ldots \ldots \ldots .140$ 


\section{List of Figures}

Figure 1.1 Conceptual Model of a Manufacturing Enterprise $\ldots \ldots \ldots \ldots$

Figure 1.2 Major Components of a Manufacturing Enterprise $\ldots \ldots \ldots$

Figure 1.3 Interdependent Domains in a Manufacturing Enterprise $\ldots \ldots \ldots$

Figure 1.4 Scheer's CIM Model $\ldots \ldots \ldots \ldots \ldots \ldots \ldots$

Figure 2.1 IBM CIM Architecture. . . . . . . . . . . . . . . . 13

Figure 2.2 Hierarchical Organization of the AMRF Model $\ldots \ldots \ldots \ldots$

Figure 2.3 CIM-OSA Integrating Architecture (From: Rembold et. al 1993). . . . . . 21

Figure 2.4 Example "Borrow Book" Shows Different Layers of View . . . . . . . 31

Figure 2.5 ANSI/SPARC 3 Schema Architecture. . . . . . . . . . . . . . 32

Figure 2.6 Student-Class Example by Using an Entity Relationship Diagram. . . . . 33

Figure 2.7 Student-Class Example by Using an IDEF1X Diagram. . . . . . . . . . . 34

Figure 2.8 ISO-OSI Reference Model for Open Communication Systems. . . . . . . 40

Figure 2.9 Subnetwork-based Network Supporting the SME Model . . . . . . . . 42

Figure 2.10 Conceptual Network Levels in a CIM Environment. . . . . . . . . . . . 43

Figure 2.11 MAP/TOP 3.0 Architecture $\ldots \ldots \ldots \ldots \ldots \ldots \ldots$

Figure 3.1 Possible Dataflow between Sample Applications. . . . . . . . . . . . . 47

Figure 3.2 Layout Representation in QSOM. . . . . . . . . . . . . . . . . . . . . . . 49

Figure 3.3 QSOM Final Layout Printout. . . . . . . . . . . . . . . . . . . . . 49

Figure 3.4 IDEF1X Data Model of QSOM's Layout Tool. . . . . . . . . . . . . . 50

Figure 3.5 Simulation Compilation, Linking, and Execution Procedure. . . . . . 52

Figure 3.6 Sample Model, Experiment, and Report of SIMAN. . . . . . . . . . 53

Figure 3.7 Entity-Relationship IDEF1X Data Model of SIMAN Experiment. . . . . . 54

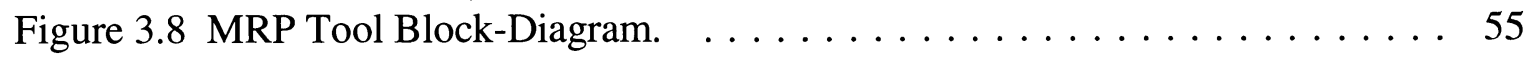

Figure 3.9 Partial Entity-Relationship IDEF1X Data Model of MRP Tool. . . . . . . 56 
Figure 4.1 Integration of Three Applications through Integration Extensions. . . . . 59

Figure 4.2 Basic Integration Extension Block-Diagram. . . . . . . . . . . . . 60

Figure 4.3 IDEF1X Extension Example. . . . . . . . . . . . . . . . . . . . 64

Figure 5.1 Level 0 Data Flow Diagram $($ DFD0) $\ldots \ldots \ldots$. . . . . . . . . . 68

Figure 5.2 Level 1 DFD Shows Data Flow between Main IX Modules. . . . . . . . 69

Figure 5.3 IX Network Layer Model. . . . . . . . . . . . . . . . . . . . 72

Figure 5.4 IX Sender and Receiver Communication. . . . . . . . . . . . . . 75

Figure 5.5 IX Receiver State-Event Diagram. . . . . . . . . . . . . . . 76

Figure 5.6 Example IX Communication Session. . . . . . . . . . . . . . . . . 80

Figure 5.7 Tool Access Module Components. . . . . . . . . . . . . . . . . 85

Figure 5.8 Link Database Example Entries. . . . . . . . . . . . . . . . . . . . 89

Figure 6.1 IX User Interface Structure. . . . . . . . . . . . . . . . . . 94

Figure 6.2 Screenshot of IX Main Card. . . . . . . . . . . . . . . . 95

Figure 6.3 Subscription Card. . . . . . . . . . . . . . . . . 97

Figure 6.4 View Public-Database Card. . . . . . . . . . . . . . . . . . . . . 99

Figure 7.1 Example Product Component Tree. . . . . . . . . . . . . . . . . 102

Figure 7.2 Example Product Routing through Departments. . . . . . . . . . . 103

Figure 7.3 Shared Data between Sample Applications. . . . . . . . . . . . . . . . 104 


\section{List of Tables}

Table 5.1 Network Access Interface $\ldots \ldots \ldots \ldots \ldots \ldots$

Table 5.2 IX Protocol Commands $\ldots \ldots \ldots \ldots \ldots \ldots$

Table 5.3 IX Protocol Reply Codes $\ldots \ldots \ldots \ldots \ldots \ldots \ldots$

Table 5.4 Data Description Language $\ldots \ldots \ldots \ldots \ldots \ldots \ldots \ldots$

Table 5.5 Data Manipulation Language $\ldots \ldots \ldots \ldots \ldots \ldots$

Table 5.6 List of Possible DDL and DML Errors . . . . . . . . . . . . . . 84

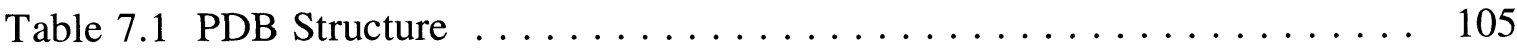

Table 7.2 PDB Structure of Simulation Tool $\ldots \ldots \ldots \ldots$

Table 7.3 PDB Structure of MRP Tool $\ldots \ldots \ldots \ldots \ldots$ 


\section{Introduction}

To understand the following discussions and concepts, this introduction will give the reader a sufficient insight into the topic of integrated information modeling for manufacturing systems. It might be more than expected from a common introduction, but the integration of so many different systems requires a comprehensive and broad knowledge of the key components as well as the entire system in which they operate.

The first section covers manufacturing systems in general. The production flow and key components of a manufacturing system are briefly reviewed and their corresponding computer aided technologies are shown. After explaining the elements of a manufacturing system it becomes evident that some integration of these 'islands of automation' is desirable. The idea of integration is therefore addressed in a section about concepts of Computer Integrated Manufacturing (CIM). A survey of enterprise and data modeling techniques presents the means of modern systems description. 


\section{Manufacturing Systems}

The manufacturing environment has dramatically changed in the last few years. Worldwide competition among manufacturers and the development of new manufacturing technologies have contributed to today's competitive situation in many manufacturing areas. This section gives a brief overview how manufacturing systems have evolved to today's degree of automation.

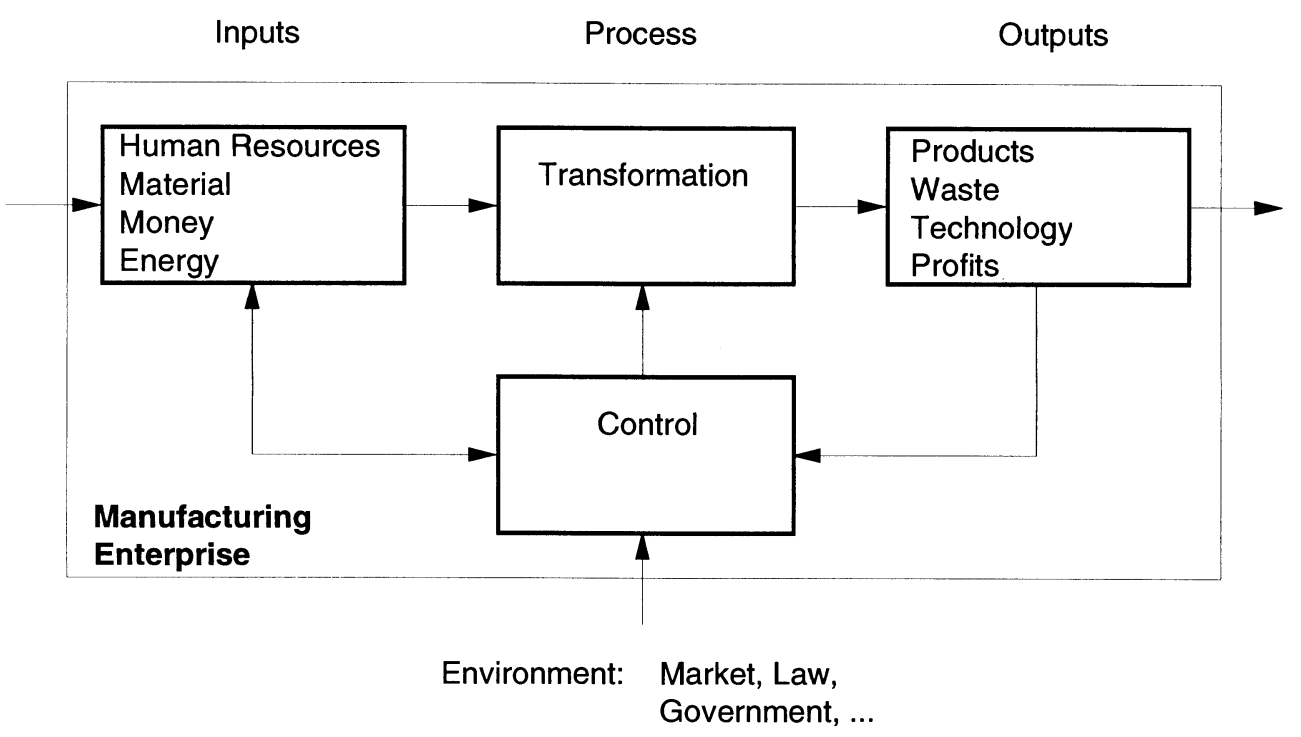

Figure 1.1 Conceptual Model of a Manufacturing Enterprise (From: Compton 1987).

Compton's model gives a good representation of manufacturing enterprise boundaries and goals (see Figure 1.1). Several inputs are necessary for the transformation process that produces desired goods and profit. Depending on the transformation process other outputs like waste or new technologies may also be produced.

The system is kept in balance by various feedback and control actions. For 
example, environment pollution laws force the company to use better technologies or change the physical location of the production. This model shows the general data flows within a manufacturing system. However, for our discussion we will not address human resources, money, or waste issues.

This conceptual model exhibits the interfaces to the environment plus the feedback/control mechanism. There are numerous additional elements necessary to run a manufacturing enterprise and keep the conceptual model in balance. Figure 1.2 shows major elements (shaded elements are manufacturing related) found in a typical manufacturing enterprise and lists also typical computerized applications:

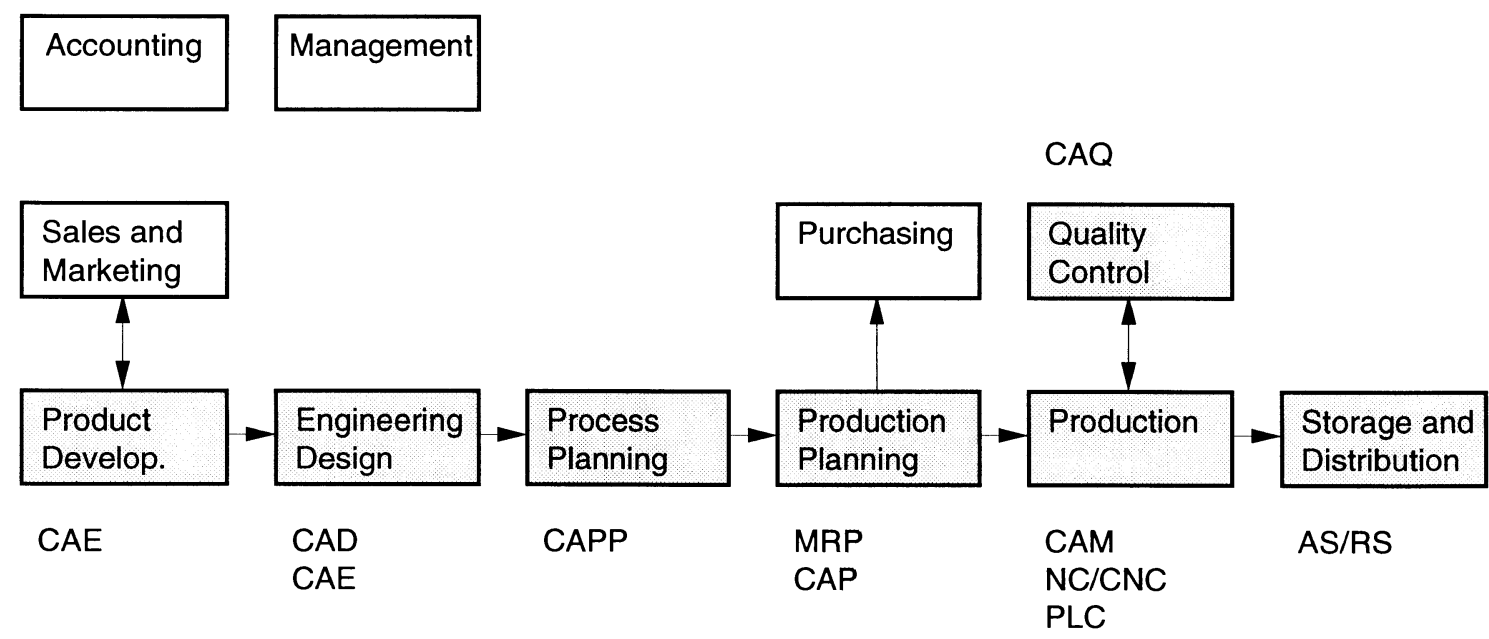

Figure 1.2 Major Components of a Manufacturing Enterprise.

Production flow is initiated with product development, marketing and sales departments. Product design makes use of CAD (2D and 3D solid modeling) and other systems such as Mass Transfer and Finite Element Method Analysis. These techniques are often referred as Computer Aided Engineering (CAE) 
techniques. The methods and tools used for product design usually depend on the product.

Process Planning is the transformation of design specification into the operating instruction necessary for manufacturing. Therefore, process planning links product design and production. Although Computer Aided Process Planning (CAPP) didn't receive much attention until about ten years ago, high technologies (such as Expert Systems, Knowledge Based Systems, Neural Networks, and Genetic Algorithms) have been applied in the development of CAPP.

The production stage includes the operations necessary to produce goods. Computer Numerical Control (CNC) machines, robotics, Programmable Logic Controller (PLC), inventory management systems, shop-floor data collection systems are examples of widely used systems. Computer Aided Quality (CAQ) assures and controls throughout product quality.

Distribution is generally responsible for acceptance, storage, and dispatch of finished goods. Modern management methods require manufacturing enterprises to keep finished goods inventory as small as possible. Distribution centers are used for storage by employing automated storage and retrieval systems (AS/RS).

Figure 1.2 can be split in three domains that work simultaneously: 1) engineering, 2) production, and 3) management. Figure 1.3 depicts the three domains and data flows among them. Engineering provides technical 
information, usually represented as drawings, to production and receives product requirements from management. Production transforms raw material to finished

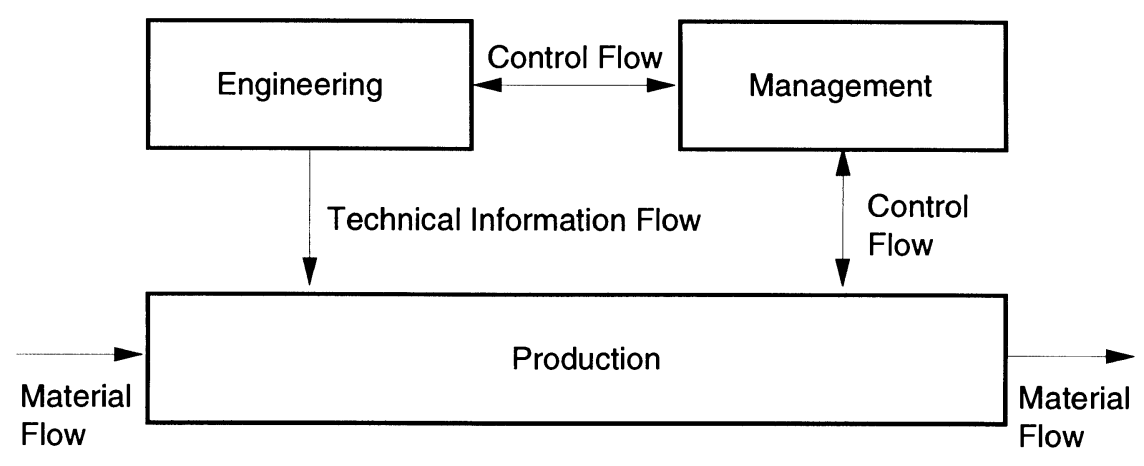

Figure 1.3 Interdependent Domains in a Manufacturing Enterprise.

goods and provides management with operational information. Management releases and monitors work order for both engineering and production.

Although many stages in a manufacturing system have been computerized, (so called C-technologies) they often remain as islands of automation. Data must be transferred manually from one system to another. However, links among similar products (e.g., CAD to CAD translation) are offered. Much effort has been made in the few past years to integrate these system with a concept called Computer Integrated Manufacturing (CIM). 


\section{Computer Integrated Manufacturing}

It seems that there are almost as many definitions of CIM as people writing about CIM. Of course this is an exaggeration, but it shows that people have distinct ideas of what CIM should be. Linking a CAD system with a CNC machine so that parts drawn on a drawing system are automatically produced by a milling machine can be interpreted as CIM. Indeed two systems which are integrated and exchange information without additional manual operation should be CIM. However, most researchers and engineers don't classify the above example as CIM. Although there is no exact definition for CIM, almost everybody agrees that true CIM should comprise almost every manufacturing aspect part of a company. This may include CAD, CAM, CAPP, CAE, CAQ, MRP, capacity planning, cost accounting and so on. A completely automated production by integrating these 'islands of automation' is often referred to as the 'factory of the future' (FOF).

A major problem in realizing the FOF is the lack of a clear CIM architecture. Many companies have developed hardware and software products for their CIM components. These components usually work solely with the companies proprietary products and ideas of a CIM architecture.

A major player for international standardization is the ISO (International Organization for Standardization). The ISO TC 184 committee deals with "Industrial automation systems and integration", and may be joined by anybody who is involved in manufacturing standards. This committee is composed of 5 
subcommittees and some 20 working groups. The subcommittee SC 5 "Architecture and communications" consists of 4 workgroups: WG 1 Framework for CIM systems integration, WG 2 Communications and interconnections, WG 3 Industrial automation vocabulary, and WG 4 Programming language environment. Other organizations besides ISO are trying to establish standards too, such as the IEC (International Electrotechnical Commission). The IEC increased their scope to include shop-floor communications, which is a basic part of a CIM architecture (Dato 1991). Despite the various available CIM definitions and architectures, this thesis will provide an example of integration of various manufacturing components. A popular model in Europe has been developed by Scheer, which is often referred to as the "Y-model" (Figure 1.4). It distinguishes between organizational planning functions (left fork) and technical functions (right fork) which make use of several databases (Scheer 1989).

There are other models available, mostly developed by large traditional computer companies such as IBM, Siemens, DEC, or Hewlett-Packard (Pato 1990). From Figure 1.4 one can see that the most important part of a CIM system is the databases and networks communication. The following two sections explain the roles of both databases and communication.

Product designs, bills of material, orders, inventory, machines capabilities, and personnel are just a few examples of items stored in these databases. The stored data changes over time and should always reflect the current situation of the enterprise. Most CIM components maintain their own database and information is shared either automatically over a network or manually by hand. 


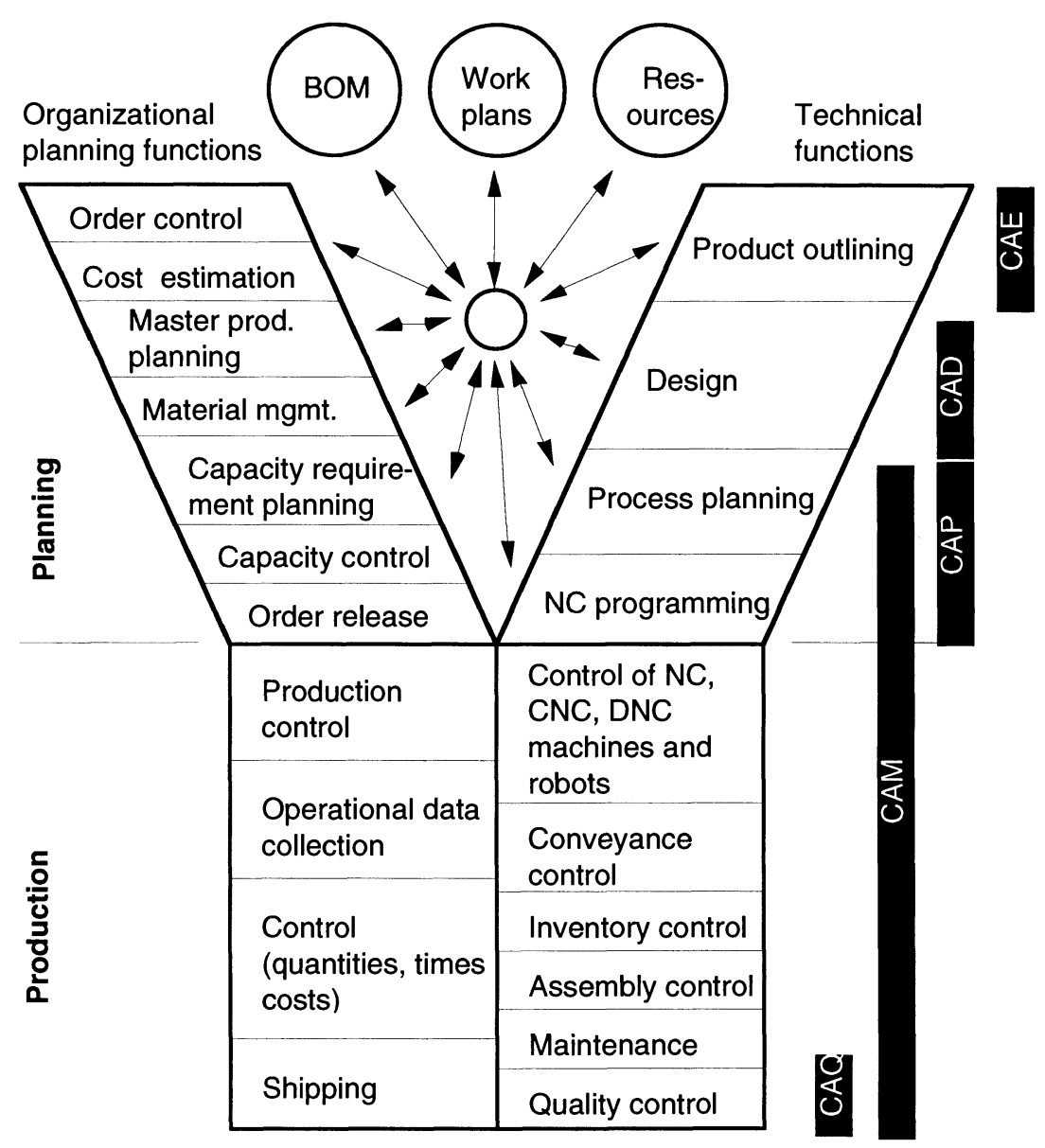

Figure 1.4 Scheer's CIM Model.

We have already mentioned that many computer applications in manufacturing have been referred to as 'islands of automation'. To establish some connection among those applications it is necessary to introduce network topologies and communication models. The communication abilities of CIM system components depend on vendor. While some products offer simple file import and export features, other products support full integration over popular network environments. Industry standard networks and manufacturing protocols will be discussed in the literature review section. 


\section{Statement of the Problem}

Previous sections explained components within a manufacturing system and showed a need for integration. Such an integration involves data exchange among the CIM components in an efficient and effective manner. The goal of integration in an enterprise is to consistently deliver the right information to the right people at the right time in the right format, regardless of their physical location.

Several integration models and techniques have been published by vendors, standardization institutes, and universities in the past years. Yet, a considerable amount of work has been done in integration of manufacturing databases.

A comprehensive integration of different components may involve many problem issues such as:

- the usage of common communication protocols

- the same data distributed on different components (redundancy)

- the same data on different systems with differing access names (semantic redundancy)

- the usage of data translators to alter data format from one component to another

- the inability to track consistency data flow in both directions

- the component's ability to share (export or import) data with other systems

This thesis will address many of the problems listed above, however, it was not possible to cover all possible issues of integration. 


\section{Approach}

The general procedure of this work was broken into three steps:

1. Selection and analysis of sample applications,

2. Establishment of an integration concept, and

3. Implementation of an integration for the selected sample applications.

Three sample applications were selected to demonstrate the proposed integration model. All applications are representative of those used for planning, manufacturing layout and production. A data model was constructed for each application. The normalized data model represents the application's data in a neutral format. This information is then shared with other applications by using network services. The integration implementation specifies communication, database formats, translation mechanisms, and application access methods.

Finally the proposed integration concept was implemented for the three sample applications to prove the suggested concept. 


\section{Literature Review}

The previous chapter introduced briefly the structure and components in a manufacturing system. The literature review shows what has been done in the areas of manufacturing databases and communication for manufacturing environments. A discussion of enterprise modeling, some existing CIM models, and other integration methods will be included in the literature review. 


\section{Existing CIM Models}

Many CIM models have been developed by traditional computer manufacturing companies, standardization institutes, and universities. IBM, Siemens (Rembold et al. 1993), Digital Equipment Corporation (Rembold et al. 1993), Hewlett-Packard, and Asea Brown-Boveri are just a few well-known companies that offer CIM solutions. A selection of other organizations and institutes with CIM model recommendations include Society of Manufacturing Engineers (SME) (Pimentel 1990), Advanced Factory Management System (AFMS) by Computer Aided Manufacturing Inc.(CAM-I) (O’Grady 1986), Advanced Manufacturing Research Facility (AMRF) by the National Institute of Standards and Technology (NIST) (O’Grady 1986), ESPRIT CIM-OSA model, and the Amherst-Karlsruhe CIM model (Rembold et al. 1993).

A few CIM models will be discussed as representatives of the list above in the following section. The discussion focuses on the structure and strengths of each model.

\section{IBM CIM Architecture}

IBM has been offering CIM solutions for a long time. They began in the early 1970s with the development of a CIM concept that included all major planning and control activities of manufacturing. IBM has a vested interest, it not only sells CIM solutions, it implements CIM in its own plants world-wide. 
IBM's CIM architecture (Figure 2.1) consists of three key components (Ganguin 1990):

- communication - distribution of data,

- database management - definition, storage, and usage of data, and

- presentation - presentation of data to user and machine.

By using these basic components the CIM architecture offers a consistent foundation of product, process, and business data of the company.

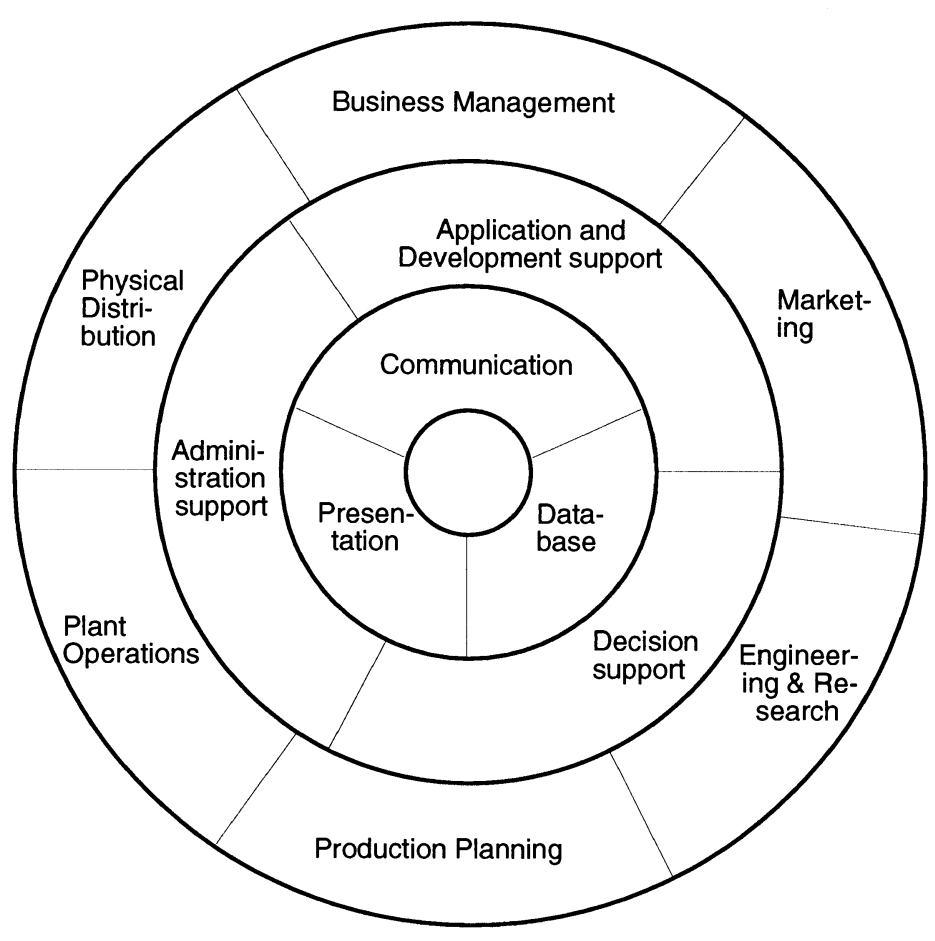

Figure 2.1 IBM CIM Architecture.

Communication or the delivery of data to user, applications, and machines is a critical issue in a CIM architecture. Today's manufacturing environments incorporate a broad range of computer systems, technologies, system architectures, operating systems, and applications which must be connected to 
each other. The numerous data formats and descriptions makes the communication for users and machines even more difficult. IBM attempted to overcome this problem by developing the System Network Architecture (SNA) (Cypser 1991). SNA allows communication between other systems and IBM systems and has become a standard in many manufacturing environments for host communication. Distributed Automation Edition ${ }^{\mathrm{TM}}$ (DAE) is software product that includes the following communication functions for manufacturing:

- definition and administration of networks

- logical assignment of manufacturing equipment

- administration of program libraries

- queue management and distribution of messages

- preparation of monitor procedures

- monitoring of manufacturing cells.

DAE also supports several communication standards like SNA connections with host systems, IBM PC networks, Token Ring protocol, and the Manufacturing Automation Protocol (MAP).

The second component, database management, defines the data structure, database links, physical location of the data, and data access rights. The many different databases, data formats, data storage and access techniques in today's environments increase the complexity of data integration. Some standards, however, like the Structured Query Language (SQL) are enabling applications and users to access relational databases from different vendors. Three databases can be identified in IBM's CIM architecture:

- a data repository 
- an enterprise data storage

- local data storage.

IBM implemented a CIM Communications and Data Facility (CIM CDF) that runs on a relational SQL database and provides the following repository functions:

- transformation of data in a format selected by the user

- storage of CAD, CAM, PPC, and other data

- adding of attributes to CAD/CAM data from a user's view

- queries of data and attributes of any kind.

CIM CDF offers communication features for transportation of data between repository and CAD/CAM components.

The presentation component provides data to users and machines in an appropriate format. Usually data presentation is done by using terminals that can be of various technologies. The same information may be treated different for each application. For example, the same production part may be displayed in a CAD drawing and in the bill of materials (BOM) of the process planning application.

The IBM CIM architecture offers a comprehensive model for enterprise system integration. This includes not only manufacturing functions, but also considers business management, marketing, and product distribution. Since many functions rely on proprietary IBM technologies and products it is not very clear how easy other CIM components can be integrated. This problem may prevent other manufacturers from integrating more sophisticated CIM applications in an existing manufacturing systems. 


\section{NIST-AMRF Hierarchical Model}

The Automated Manufacturing Research Facility (AMRF) was established by the National Institute of Standards and Technology in 1982. The objective of the AMRF is to develop hardware and software standards for computer controlled manufacturing systems. The AMRF architecture proposes to break down complex planning and control functions into a hierarchy of levels (Figure 2.2).
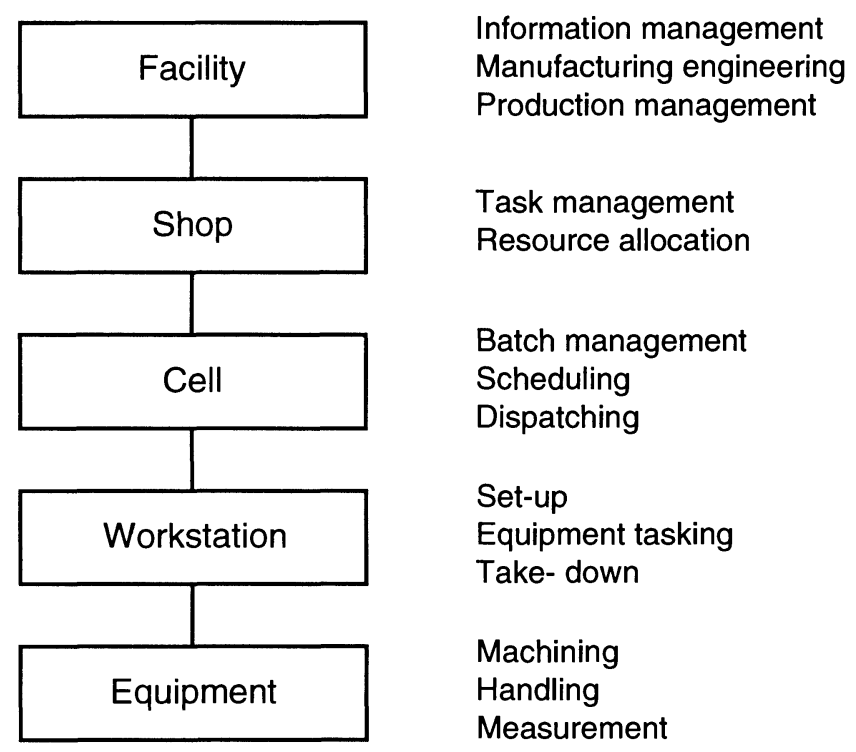

Figure 2.2 Hierarchical Organization of the AMRF Model (From: O'Grady 1986).

The facility level includes process planning, production management, and information management (with links to financial and other administrative functions). At the shop level, systems manage the coordination of resources and jobs on the shop floor. A group technology (GT) classification scheme is used to group jobs into part batches at this level. A feature of this stage is the 
introduction of the concept of a virtual manufacturing cell. Virtual manufacturing cells comprise machines which can be interconnected in a dynamic fashion. Other functions at this level include allocating tooling, jigs, fixtures and materials to specific workstation/job combinations. The activities are reevaluated based on feedback from the cell level and commands from the facility level.

The cell level incorporates scheduling and controlling of jobs and material handling within the cell. The workstation level handles the arrangement of the sequence of operations to complete the jobs allocated to the cell control system. A workstation can be a robot, a machine tool, a material storage, or a control computer.

Finally the equipment level consists of the controller for individual resources such as tools, robots, material handling systems and associated sensors and actuators (Pimentel 1990).

While the IBM CIM architecture seems to be more business oriented, the AMRF from NIST emphasizes more on the actual manufacturing part. The planning and control hierarchy includes information and product management, however, most layer deal with the actual production. For example, the interaction and integration of low-level devices such as robots and machine tools is more detailed specified in the ARMF model. The hierarchical approach decomposes the overall system into a series of levels that are more modular and controllable. Since the AMRF is a research facility it cannot be bought and easily implemented like a system from Siemens or IBM. However, models such 
as the AMRF come up standards and methodologies in the integration of various CIM applications that can be used by CIM implementors.

\section{CIM-OSA Model}

The AMICE project (reverse acronym for European Computer Integrated Manufacturing Architecture) was launched by the European ESPRIT program in 1984. Twenty-one companies (CIM users, implementors, and research institutions) from seven EC countries participated in this program. The result of the AMICE coalition was CIM-OSA, an Open-Systems Architecture, that provides an integrated methodology to support all phases of a CIM system lifecycle.

CIM-OSA uses a standardized set of constructs and models to create (with a series of well-defined steps) a precise model of the desired CIM system. CIMOSA takes accepted ideas and models into account: functional decomposition with System Analysis and Design Technique (SADT), information modeling with the ICAM Definition Method (IDEF1X) and Entity Relationship Diagrams (ERD), the 3-schema approach from ANSI/SPARC, various data communication and network standards (OSI, MAP, TOP) etc. Addressing the real needs of the industry as well as supporting multi-vendor system environments with new and/or existing components are two key elements of the open-systems architecture. For this purpose CIM-OSA defines a framework for open modeling that provides (Jorysz and Vernadat 1990):

- a frame for standardization 
- a limited but expandable set of basic modeling constructs

- a representation formalism

- a set of application methodologies.

The CIM-OSA concept consists of two environments: the enterprise engineering and the enterprise operation environment. The engineering environment provides the reference architecture and resources to structure and set up an enterprise model. The designer will be able to consult the reference architecture and to generate a particular enterprise model (particular architecture). Diverse strategies may be modelled and simulated to come up with an optimal solution. CIM-OSA defines four different views to specify all aspects of an enterprise:

- Function view

- Information view

- Resource view

- Organizational view

The function view describes the static and dynamic functional properties of the enterprise. It does that through three steps. The function view specifies what is required in the requirements-definition model (this includes the required structure, content, behavior, control, and required capabilities.) It then specifies how these requirements need to be implemented, namely the detailed design of the functional system, in the design-specification model. Finally the function view describes the actual implementation of the physical and decision system in the implementation-description model in terms of manufacturing technology and information technology. 
The information view describes the information needed for each function. The information-modeling paradigm in CIM-OSA consists of:

- a frame for information modeling based on the object-entity relationship attribute (OERA) approach,

- the ANSI/SPARC 3-schema approach,

- an information modeling and analysis methodology which is part of the CIM-OSA system life-cycle, and

- a representation formalism defining the modeling constructs.

The OERA is used for high-level semantic modeling at the requirements definition level. The more precise design specification level is modeled with a data-oriented semantic model which is based on the entity relationship attribute approach. Finally a translation mechanism transforms entity relationship attribute models into relational, hierarchical or network database structures. More details about the CIM-OSA information modeling can be found in (Jorysz and Vernadat 1990).

The resource view contains different levels of abstraction of the data-processing resource of the enterprise. The central part of the CIM-OSA integrated dataprocessing environment (IDPE) is the CIM-OSA integrating infrastructure (IIS) which provides services to integrate all specific application processes into one cooperating system. This process is applicable to this work because it shows realization and execution of models in heterogeneous environments. 


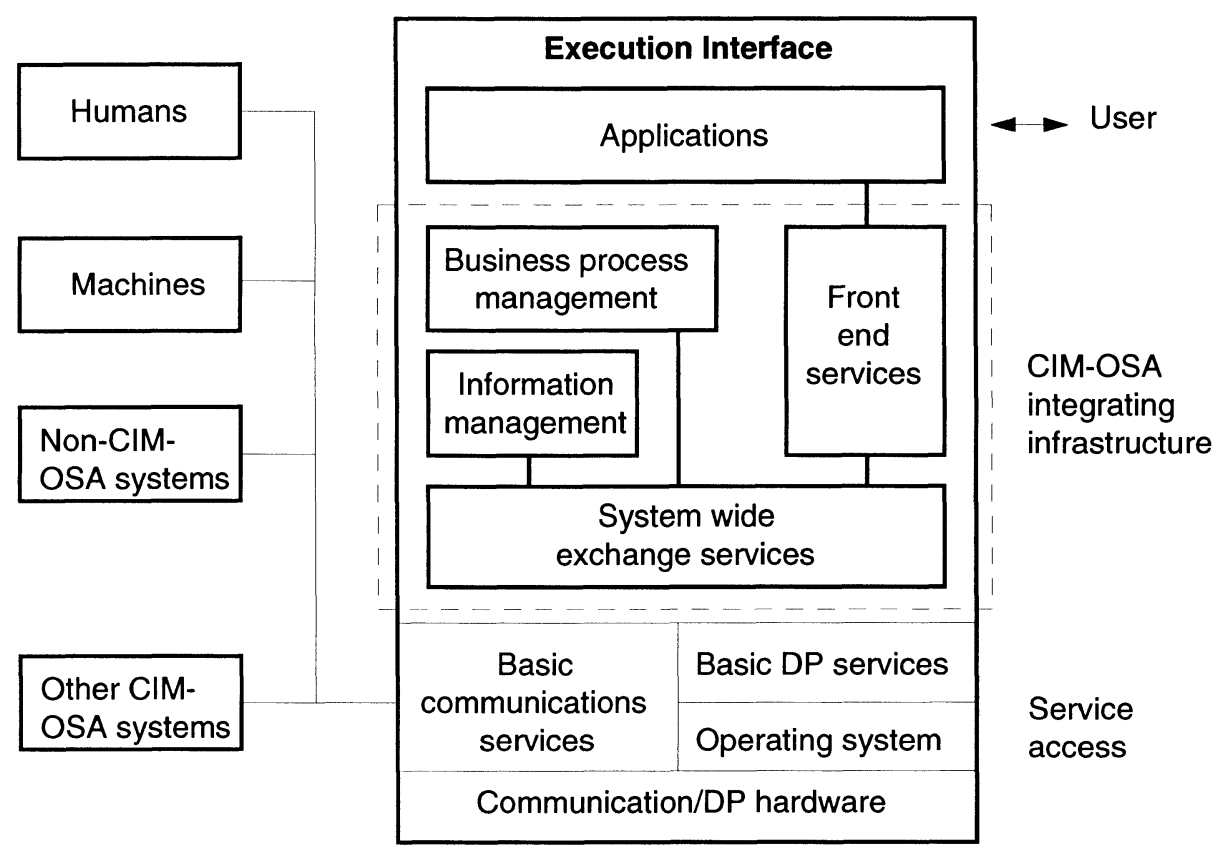

Figure 2.3 CIM-OSA Integrating Architecture (From: Rembold et. al 1993).

The IIS (Figure 2.3) provides both general-purpose services for manufacturing systems and integrating facilities for application specific functions. The frontend-services are responsible for the interaction between the individual specified functional entities and their common service entities of the IIS. There are four types of front-end-service entities:

- the machine front-end-service integrates machine specific functions like robots, NC machines, PLCs, etc.,

- the human front-end-service integrates human work,

- the application front-end-service integrates application specific data processing, functions such as CAD, CAM, CAP, MRP, etc., and

- the data-management service integrates data storage including their database management systems. 
Typical functions of front-end-services are establishment of communication channels, loading programs, controlling programs, testing of operability of devices and programs, and keeping a repository of dynamic status information of both devices and programs.

The business process management includes three types of services: 1) business process control services, 2) activity control service, and 3) resource management service. The business process control service dispatches the execution of sub-business processes and enterprises activities by interpreting their procedural rule sets. The activity control service controls the execution of enterprise activities by dispatching functional operations which are carried out by the installed capabilities. Resource management dynamically schedules the resources used by the enterprise activities to perform their functional operations.

Information Management handles all data affairs on behalf of other services of the IIS. This includes services for locating, addressing, storing, retrieving, and converting data and for management of consistency, redundancy, integrity and access rights.

The system-wide exchange services belong to the communication services and are the basis for implementation of all protocols defined among other services of the IIS. The communication services are responsible to provide location independent data access, to establish transaction channels, to manage synchronized data transfer, and to perform data recording for recovery of pending requests. The IIS can be implemented in centralized and distributed facilities. The services entities will be incorporated as a system of cooperating 
service agents on the distributed systems. An agent protocol links the service entities of each system together.

CIM-OSA is a comprehensive model that includes many standards used in industry. For example, previously developed functional process descriptions (with IDEF1X) can be easily blended into a new CIM-OSA design. The CIMOSA concept is product independent and can be implemented as a new system as well as integrated in an existing environment. This is possible through the IIS which enables communication services to non CIM-OSA systems. However, CIM-OSA involves so many components that it is difficult to implement every part of it. Therefore, not all CIM-OSA implementations will include all feasible features, which is possible through the open system architecture. Unlike many other CIM models and architectures proposed by research institutions and universities, CIM-OSA is mainly supported by companies that use CIM for their own manufacturing production.

The three presented CIM models have different architectures and therefore they have distinct advantages and disadvantages. They show that there is no 'correct' integration architecture or model. The 'right' CIM model depends significantly on the existing environment or organization. 


\section{Enterprise Modeling}

An enterprise model is a representation of the enterprise and how it works. We have already mentioned that computer supported enterprise functions (CAD, CAM etc) were created independently and often do not share the representation. This problem has resulted in different representations of the same enterprise knowledge. It is common for enterprises to have concepts that denote the same thing but have different names. The representations are also often defined without an adequate specification of what the terminology means (semantics) which leads to inconsistent interpretation and use of knowledge. Many enterprise models have been developed such as the Toronto Virtual Enterprise (TOVE), Institute für Wirtschaftsinformatik (Scheer), Purdue Enterprise Reference Architecture (Nof 1993), CIM-OSA (which is also considered to be an enterprise model), and Graphes à Résultates et Activités Interreliés (GRAI) from the Universite de Bordeaux (Nicholson 1991).

A list of characteristics of representation for an enterprise model was given by (Fox et al. 1993):

- Generality: To what degree is the representation shared between diverse activities such as design and marketing ?

- Competence: How well does it support problem solving ?

- Efficiency: Space and inference. Does the representation support efficient reasoning, or does it require some type of transformation?

- Perspicuity: Is the representation easily understood by the users ? Does the representation "document itself"?

- Transformability: Can the representation be easily transformed into another 
more appropriate for a particular decision problem?

- Extensibility: Is there a core set of ontological primitives that can be partioned or do they overlap in denotation?

- Granularity: Does the representation support reasoning at various levels of abstraction and detail?

- Scalability: Does the representation scale to support large applications?

Another important issue in enterprise modeling is the consistency of data. It is difficult to keep the enterprise knowledge globally consistent. This means that inconsistency has to be managed so that it does not unfavorably affect operations. I propose that intelligent communication among consistent subdomains is the solution to this problem.

\section{Elements of Enterprise Modeling}

An enterprise model typically includes a functional model to describe processes that create, modify, or destroy entities within the enterprise. Some processes may be subdivided for a more detailed analysis or presentation. The IDEF0 notation has been used increasingly for functional modeling. Other techniques for process modeling are Data Flow Diagrams (DFD), Petri nets, industrial process flow charts, and other specialized notations. Another key element in an enterprise model is the collection of resources needed to execute processes. Resources are often treated different from inputs, which are either consumed during a process execution (e.g., energy) or are incorporated into the outputs of a process (e.g., paint). 
An Integrated Framework for Enterprise Modeling (IFEM) has been proposed by (Malhorta and Jayaraman 1993) that extends the IDEF methodology. The two primary modeling components of IDEF0 are functions (represented by boxes) and the data and objects that inter-relate those functions (represented by arrows). In addition to the IDEF0 language, this methodology also prescribes procedures and techniques for developing and interpreting models, including ones for gathering data, diagram constructing, conducting review cycles, and creating documentation. See Federal Information Processing Standard 183 (FIPS 1993) for a detailed description of the IDEF0 technique. IFEM tries to overcome shortcomings of IDEF by integrating function, information, and dynamic models into a single cohesive system representation. The static architecture is built by integrating information and function models. Input, outputs, controls, and mechanisms for each function related in terms of entities defined in the information model are used to model the static architecture. The dynamic model is developed as an extension of the full static architecture. IFEM provides also different means for consistency checking between the integrating models.

Functional models are one of several models used for enterprise modeling. Entity models also known as conceptual models, data models, or information models are employed for structuring the entity descriptions and the relationships among entities in an enterprise model. A number of information modeling techniques are available including IDEF1X and Entity Relationship Diagrams (ERD). 
Another modeling technique is the integrated multi-view system description approach to approximate factory modeling by (Wang et al. 1993) also called IDEM (Integrated Design and Modeling) approach. IDEM sits on top of LOOPS which is an integrated knowledge engineering language encompassing object, rule, access and procedure oriented programming environment. This technique proposes the use of three hierarchical levels of modeling:

- conceptual modeling

- approximate modeling

- detailed modeling.

The conceptual modeling level is used by the top management level from the process of structuring. This level provides a language to express abstract plans. A reference model for operational management's activities is the outcome of the conceptual modeling. From this, a complete, but approximate, factory model description is built which contains all crucial aspects of the system. However, it is approximate because details irrelevant at this level can easily confound the global image of the system. The critical operations of a particular function are analyzed through detailed modeling.

The IDEM multi-view system description is based on accepted paradigms in the field of system design and modeling. At the three levels, IDEM uses three views (function, information, and dynamic) for an integrated system description. The function view is realized through top-down function decomposition using an extended version of IDEF0. The extended version of IDEF0 includes conceptual extensions (active objects that drive an IDEF0 diagram by producing or consuming flows), syntax extensions (backgrounds, line styles, different fonts), and facility extensions (flexible labeling, page 
browser etc). The information view is focused on what functions utilize specific data. This view uses features such as generic information classes, creation of information objects, and specialization of information classes. The dynamic view represents the temporal behavior of the system by using rule-based behavioral knowledge representation. The main characteristics of this view are association of process times to system functions, creation of process events, and expression of decision-making knowledge.

Generally, enterprise models seem to be built for several purposes:

- to restructure business processes for efficiency,

- to realign organizational structures to better match business activities,

- to design, or redesign, computer applications,

- to identify redundancies and inconsistencies in the entire organization (and not just in one part of the organization), and

- to help management have a more comprehensive understanding of their business organization.

Once established, the biggest problem of enterprise models is that they are often not kept up-to-date. Enterprise models tend to sit on the bookshelf because they are not directly connected to the daily operations and/or actual applications used in an enterprise. There are many reasons why it is difficult to maintain enterprise models (Eirich 1992):

- they are too complicated to understand,

- the maintainers of an enterprise model are not always aware of changes as they occur in other parts of an enterprise, 
- the enterprise change is ongoing; enterprise models are an out-of-date "snapshot" of the organization even when they are first created, and

- there is often too much irrelevant information in many models (which is not worth maintaining). 


\section{Databases}

Databases are considered to be the heart of CIM systems. There are few components in a CIM system that do not produce or consume data. While some is temporal, much of this data needs to be stored permanently. This chapter introduces common database models, logical data models, database design, typical manufacturing databases, and the concept of integrating databases and complex information systems.

\section{Models and Schema}

For storage, retrieval and processing requirements, data can be organized in different ways. There are three common database models in use currently: 1) hierarchical, 2) network, and 3) relational. The hierarchical model is often used for simple data representation (sequential). It follows a tree structure, where a parent can have one or more children, however, a child can have always just one parent (1-m relation). Hierarchical structures do not allow m-m relationships (a child having one or more parents) which are important in practice. This problem can be solved with a network structure (not to be confused with computer networks). Networks permit relationship between any entities (m-m relations), making them complex and difficult to maintain. The third model, the relational model, overcomes the previous problem by using tables with rows representing records and columns representing fields of traditional file systems. This model was published by E.F. Codd (Zehnder 1989) in 1970 and is probably the most used database model for commercial 
applications. The relational model stores data in different tables and not the relationship between data. This means easier data storage and more effort to construct queries.

The database user and database designer have often different views of data (Figure 2.4). The logical layer sits between the user's and the computer's view. The logical layer is also called conceptual schema and should replace technical terms with precise and familiar descriptions from the user. In 1975 the ANSI/SPARC (Özsu and Valduriez 1991) study group published a report which described the "ANSI/SPARC 3 schema architecture." This architecture (Figure 2.5) defines three views of data: 1) the external view, 2) the internal view, and 3) the conceptual view. The external view (or external schema) specifies the data available for a particular user or programmer. A database can have multiple external views. The internal view describes the structures of the physical storage.

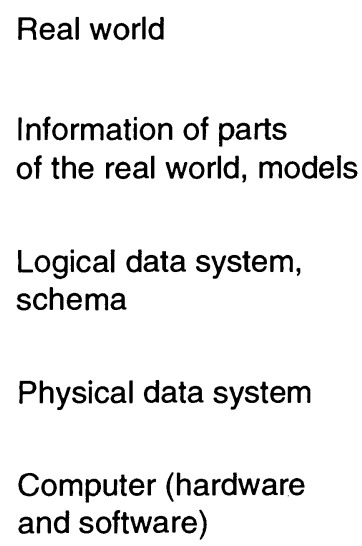

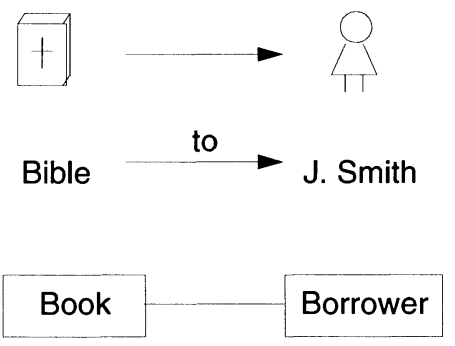

Physical data records and files

Storage media
Database

Figure 2.4 Example "Borrow Book" Shows Different Layers of View (From: Zehnder 1989). 
It is the computer's or system's view of the data. The logical layer is represented through the conceptual view. The conceptual view (or conceptual schema) describes the data independent of computer or database software that is used.

\section{Database administrator}

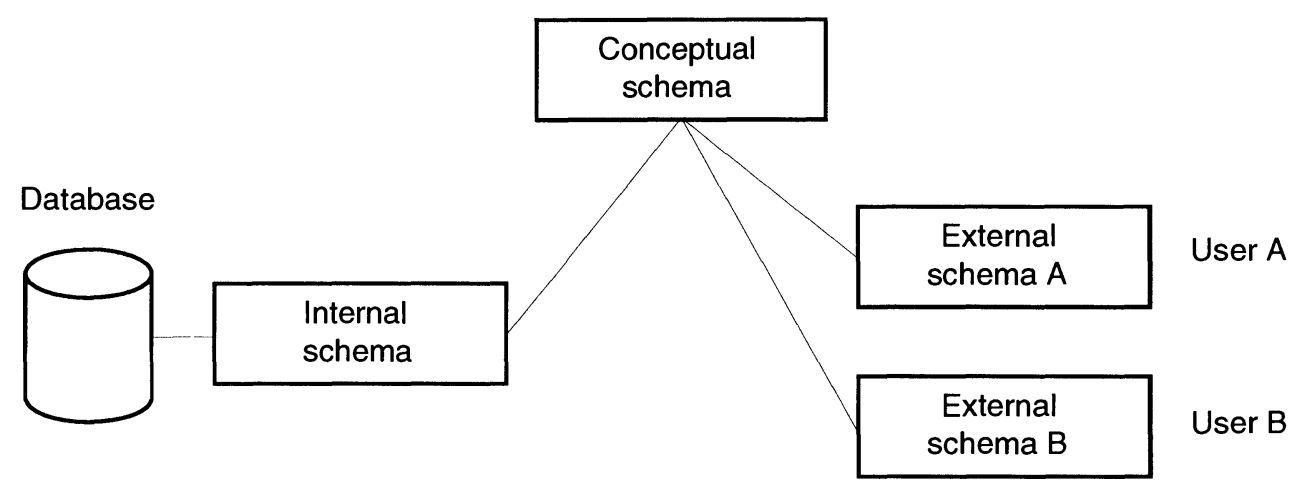

Figure 2.5 ANSI/SPARC 3 Schema Architecture.

The ANSI/SPARC 3 schema architecture serves also as a basis for many other database architectures.

\section{Information Modeling}

To model information in a database system we need tools to help us describe the data. Information modeling tools support systematic analysis of data and provide a model that can be used for redundant-free storage of data. The Entity Relationship (ER) model for conceptual database modeling was introduced by P.P. Chen in 1976. It uses rectangles to specify entities and diamond-shaped objects to represent various types of relationships. Figure 2.6 shows an ER example of students taking classes. The $\mathrm{m}$ and $\mathrm{n}$ specify the association type 
between entities. In this example students can take one or more classes (n) and classes can be taken from one or more students (m). Although attributes are included in this case, they are usually omitted in larger ER diagrams to reduce complexity. The underlined attributes represent key attributes which is used for clear identification of a database record.

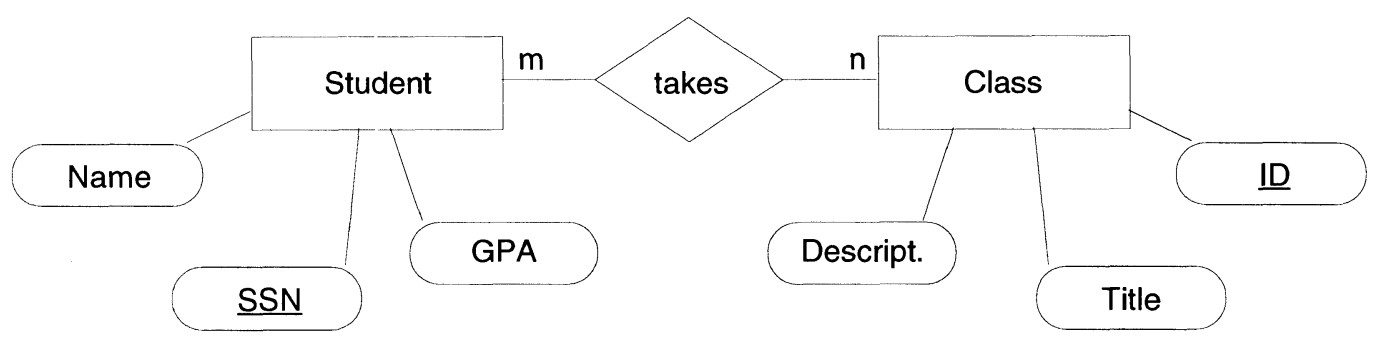

Figure 2.6 Student-Class Example by Using an Entity Relationship Diagram.

Another popular method for information modeling is the IDEF1X notation (FIPS 1993). By using IDEF1X's full decomposition methodology the information modeler will come up with a normalized data structure (3rd normalized form).

Normalization of data structures is necessary to create database tables which are free from redundancy. The ER diagram (Figure 2.6) uses a m-n relationship, the IDEF1X diagram in Figure 2.7, however, shows a normalized version of this relationship. 


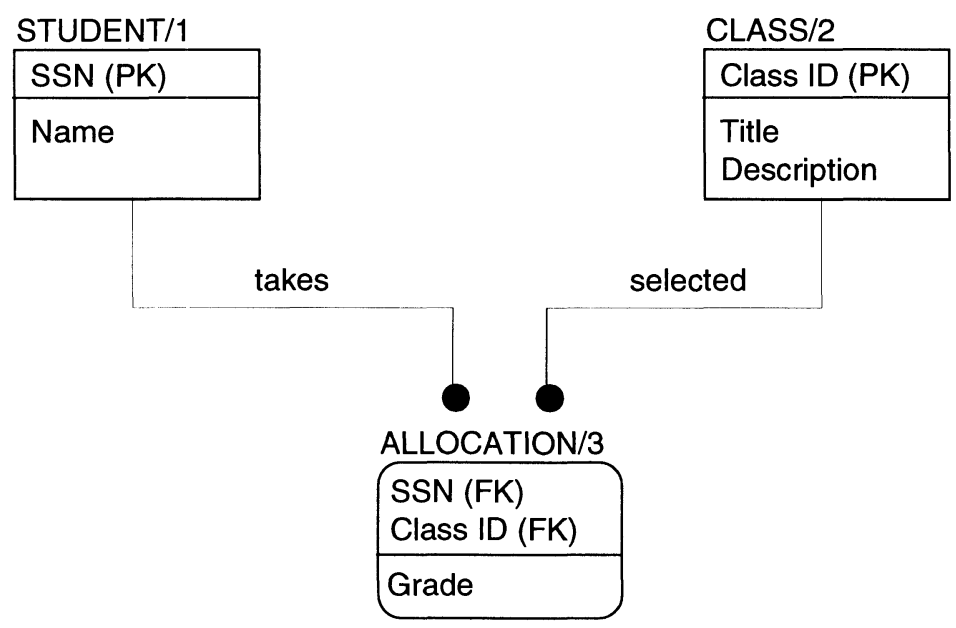

Figure 2.7 Student-Class Example by Using an IDEF1X Diagram.

\section{Database Design}

A database system may be designed either centralized or distributed. A centralized database stores all data on one database system with multiuser access on the same computer system. Centralized databases have the advantages of minimum data redundancy, high degree of data consistency, high degree of data security, and provisions for loss of data. One disadvantage of a centralized system is the availability of the system during system failures.

A distributed database system consists of multiple databases that may reside on a single computer or on multiple computers (also called multidatabase system). These systems may differ in hardware, operating and application system, and communication support. A multidatabase system is called homogeneous if all database management systems in a distributed environment are the same; 
otherwise it is called heterogeneous. The goal of a distributed database system is to improve access, data sharing, and performance of a database system while preserving the appearance of a centralized database system.

The database design issues in an distributed environment differ from those encountered in a centralized database system. Distributed database design issues include global optimization of processing and network communication. The three most common objectives of distributed database design (Teorey 1994) are the

- separation of data fragmentation from data allocation,

- control of data redundancy, and

- independence from local database management systems.

\section{Manufacturing Databases}

Most CIM components maintain some kind of storage for local data processing and for access from other components. The stored information may include drawings, designs, bill of materials, orders, inventories, machine capabilities, personnel and so on. These databases are constantly updated and therefore reflect the current situation of the facility.

A hybrid manufacturing database system may consist of the following four main-databases (Zhang and Alting 1994):

- Design database 
Consists of geometrical modeling data, bill of materials, group technology coding, etc.

- Process planning database

Contains several sub-databases such as raw material, machine tools, tooling, and process plan.

- Manufacturing database Specifies how to manufacture products or parts, and consists of NC programs, machine tools layout, tools, transfer devices, and material handling equipment.

- Production management database Contains administrative sub-databases about products, personnel, customers, suppliers, inventory status, equipment and processes, master schedule, and workshop loads.

Each database can be decomposed into further databases as necessary.

However, the information discussed above is not always easy to access from outside the particular CIM component. Some applications offer special data import and data export functions to share data and only very few applications have database-like data access functionality. Other applications do not have any specific functions to access their internal data. In this case it is necessary to investigate the application's proprietary file-formats and to develop converters to read and write the application's data. 


\section{Database Integration}

The integration of databases (and therefore communication of data between databases) has many important aspects that need to be addressed. Some issues to be considered are (Rembold et al. 1993):

- Adequacy of communication language

- $\quad$ Language uniformity

- Openness to communication

- Assurance of data integrity

- $\quad$ Provision for loss of data

- Protection from interference with parallel communicators

- Independence of physical communication media and computing systems

- Access rights

- Multiuser operation

Often the integration is simply done by static file transfers from one tool to another. If both tools do not support a standardized file format (such as DXF or IGES for drawings) additional software will be needed to extract the desired information from one tool and to arrange the data to the expected format of the other tool. This kind of integration is normally used for a fixed data exchange between two designated tools.

The file transfer method usually does not involve data exchange between more than two applications. However, one application could be the data source for many other applications. 


\section{Communication}

Communication is an important part of computer integrated manufacturing. Various communication technologies are available to meet the requirements on all levels of manufacturing integration. However, communication networks on shop floors impose different requirements than office communication networks. There are also other issues such as transmission speeds, network reliability, deterministic access which must be addressed before implementation. Many standards have been developed by companies that are widely used and considered to be industry standards.

\section{Communication Types}

The topology of a communication network has a significant impact on the operational security, capability, and installation costs. Besides simple point-topoint connections it can be distinguished between bus, ring, star, and tree topologies (Pimentel 1990). In a bus topology all stations (or nodes) are connected to a central highway. Addition or removal of nodes is very easy. There is no special need for a master (depends on the protocol used). The bus topology is probably the most used network topology. The ring topology all nodes are connected to a ring, which means that every nodes has always two neighbors. A broken connection in a ring network affects the entire network. The star topology is a centrally oriented structure. A number station are connected to one central station master which coordinates the communication between all connected stations. Since all communication must go through the 
central station the network throughput and reliability depends highly on this master station. Tree topologies are similar to star topologies, however, sub branches may be added to any station which is also its main advantage.

Access to a network is given by access procedures. Various access procedures for different needs are available. The most important access procedures for manufacturing networks are polling, time-division multiplex, carrier-sense multiple access (CSMA), and token passing.

\section{ISO-OSI Reference Model}

The standardized ISO-OSI reference model forms the fundamental structure for open systems. This model allows manufacturer independent communication through many standardized protocols and functions. The entire reference model (ISO 7498 and CCITT X.200) has been published in four parts (Dickson and Lloyd 1992):

- Basic reference model

- Security architecture

- Naming and addressing

- Management framework

The reference model (Figure 2.8) is based on four principles (Rembold et. al 1993):

- The communication functions are divided into layers.

- The services to be provided by each layer are specified. 
- Layer $\mathrm{N}+1$ which is immediately above layer $\mathrm{N}$ uses the services of the latter to implement its functions.

- The communication between the layer $\mathrm{N}$ and the participating terminals is specified by the ISO protocols.

The OSI reference model comprises seven layers. The four lower levels provide data transmission services, while the upper three layer serve the application and provide transportation of information rather than that of data.

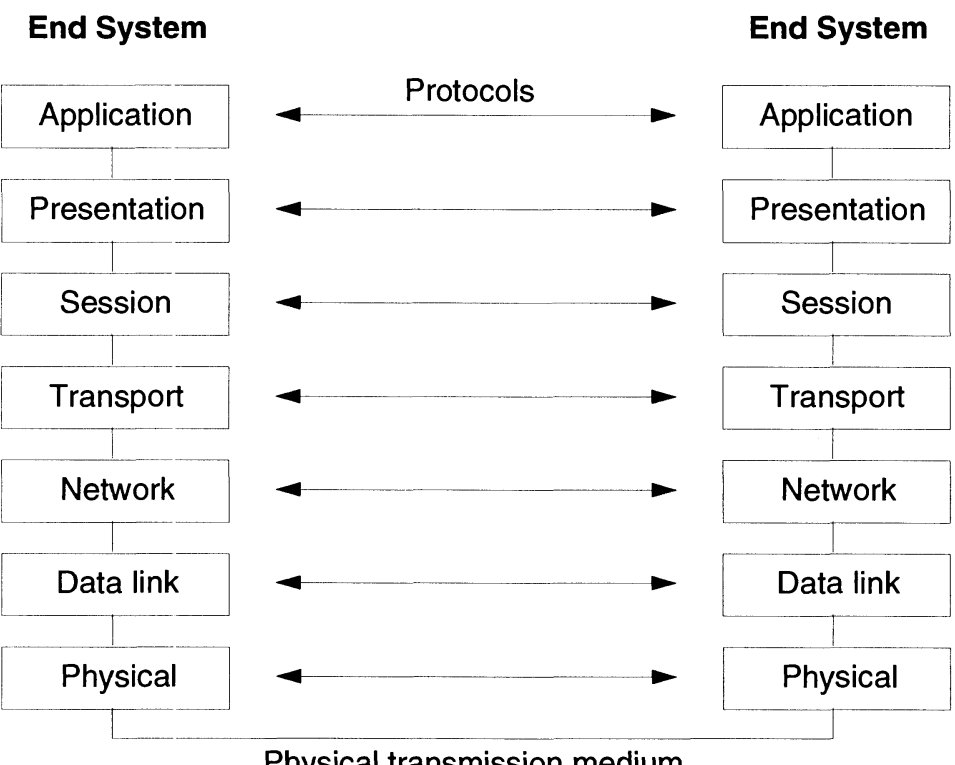

Physical transmission medium

Figure 2.8 ISO-OSI Reference Model for Open Communication Systems.

The physical layer defines electrical, functional, and mechanical characteristics (e.g., RS-232, IEEE 802 series). The data link layer provides access to the transmission media and supports secure transmission of data blocks (e.g., High Level Data Link Control (HDLC), Link Access Protocol). The network layer does the routing within the network by selecting the best path for end to end transfer of data (e.g., X.25 packet level). The transport layer provides a reliable 
logical transport connection to a target system in a network independent fashion. Packets sent through different paths over the network (by the network layer) will be assembled to logical information units. The session layer is responsible for coordination of activities between the end systems. Its functions include symbolic to real address conversion, dialog control (synchronization), and re-establishment of interrupted transport connections. The presentation layer converts system-internal data presentation into a form suitable for common interpretation by both end systems. The Abstract Syntax Notation One (ASN.1) is an ISO formal data description language for this layer. Message compression and data encryption are also located in the presentation layer. The application layer provides services to end users. Many standards have been developed for the application layer such as message handling systems (MHS, X.400), directory services (X.500), file transfer, access and management (FTAM), and others.

\section{Networks in Manufacturing}

Virtually every system in a manufacturing environment uses networks for data exchange. For example, networks transport CAD data to NC machines, make orders from the sales department immediately available to the raw material purchasing department, share the bill-of-materials (BOM) with inventory systems, and so on. Networks also grant access to common databases and other resources. 
Figure 2.9 shows an example manufacturing network using a communication backbone to interconnect subnetworks. In this case the subnetworks consist of different topologies. Each subnetwork has a gateway that allows connection to the backbone. An actual network structure depends on the overall size of an enterprise, number of stations to connect, and special requirements of particular components such as real-time ability or operation in an environment with unusual electromagnetic waves. The subnetwork approach results usually in better reliability and performance. Subnetworks continue to operate even when

Factory automation LAN

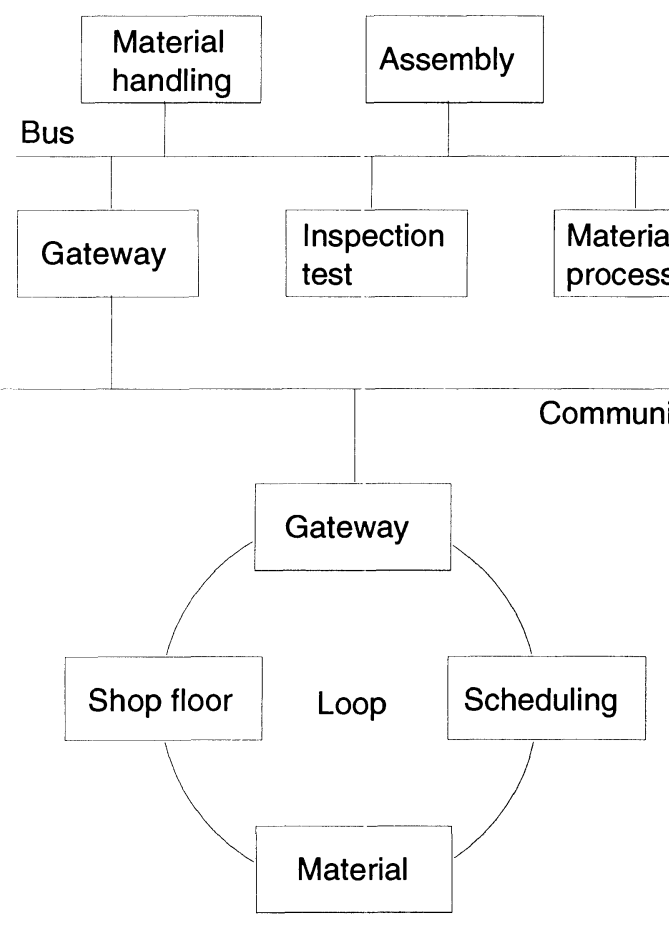

Manufacturing control LAN
Engineering design LAN

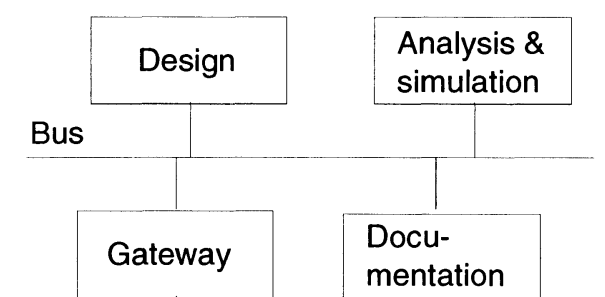

Figure 2.9 Subnetwork-based Network Supporting the SME Model (From: Pimentel 1990). 
the communication backbone or any other subnetwork goes down. A disadvantage may be delays in sending messages from a subnetwork to another subnetwork caused through gateways and backbone.

Figure 2.9 also shows the logical arrangements of components in groups such as engineering design, factory automation, manufacturing controlling, and manufacturing planning. This leads to a relationship between the control and component level hierarchies and networks supporting them. Figure 2.10 exhibits a network hierarchy that can be identified for an automated manufacturing facility.

Level
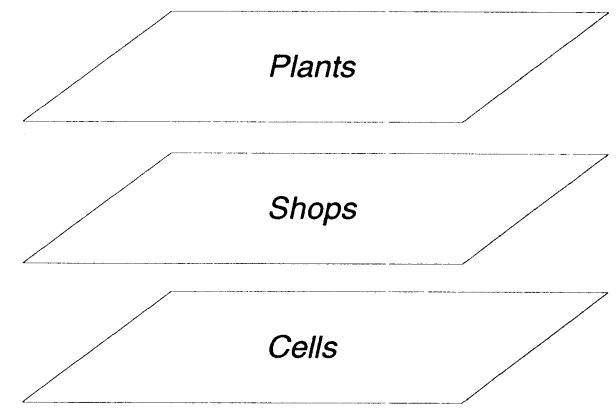

Workstations
Network

Wide Area Network

- e.g., CAD, CAP, CAE

Local Area Network

- e.g., CAP, PPC, MRP

Local Area Network,

Fieldbus

- e.g., CAM, CAQ

Local Area Network,

Fieldbus

- e.g., CAM
Performance variables of most interest

Throughput

Throughput

Response time, throughput

Response time

Figure 2.10 Conceptual Network Levels in a CIM Environment. 


\section{Manufacturing Communication Profiles}

A few standards have been developed to provide data linkage between heterogenous computer and control systems in CIM environments. Probably the most cited specification is the manufacturing automation protocol (MAP) and the technical office protocol (TOP). The MAP/TOP architecture supports open communication and make extensive use of existing standards of the OSI reference model for each layer. The current version is MAP 3.0 (Figure 2.11) which features significant enhancements compared to earlier versions. MAP 3.0 includes now the Manufacturing Message Specification (MMS) which is widely used by manufacturing companies.

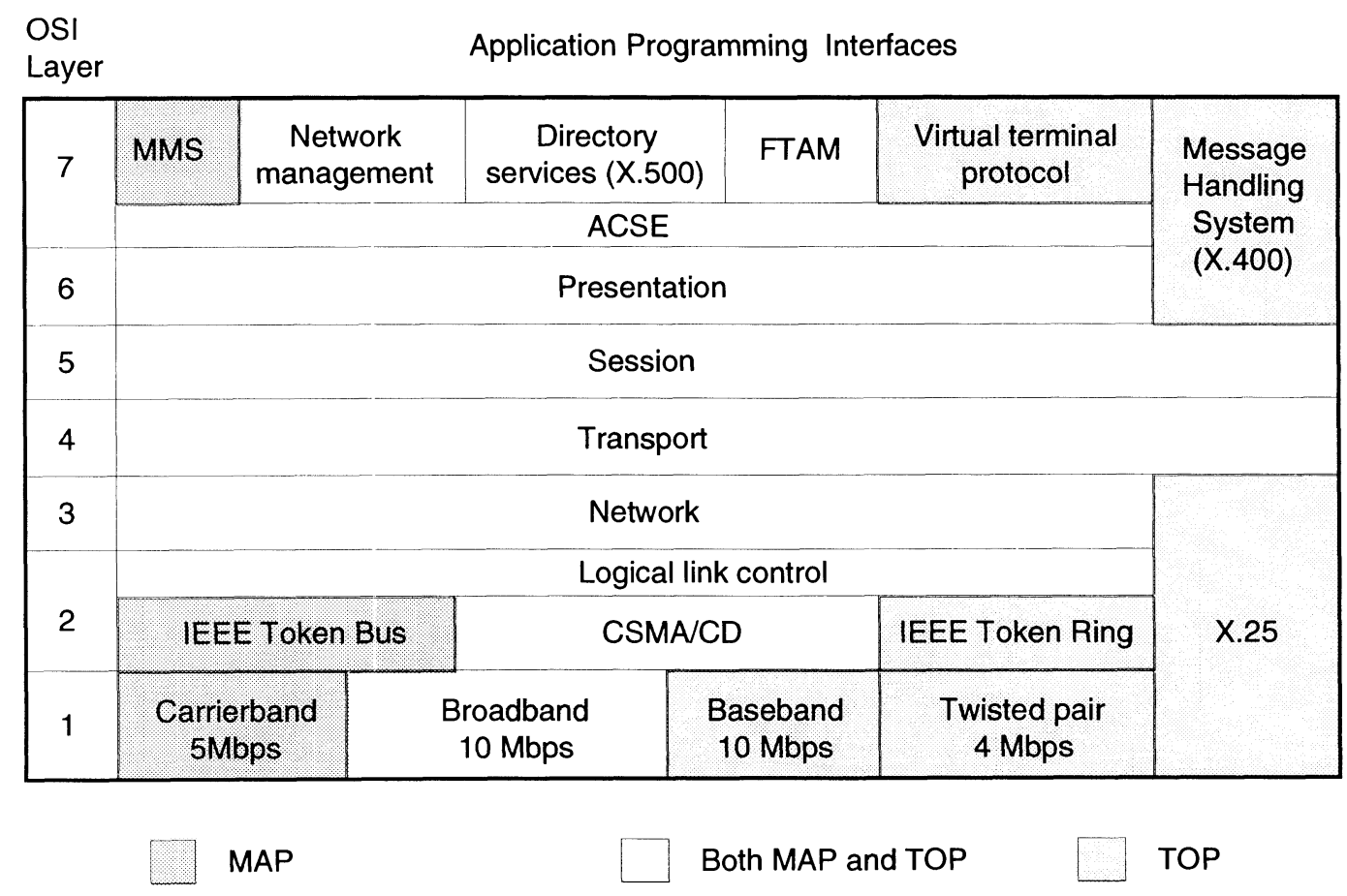

Figure 2.11 MAP/TOP 3.0 Architecture. 
MMS is an internationally standardized messaging system for exchanging data in real time and supervisory control information between networked devices and/or computer applications in a manner that is independent of: 1) the application function being performed or 2) the developer of the device or application. The messaging services provided by MMS are generic enough to be appropriate for a wide variety of devices, applications, and industries. MMS provides a rich set of services for peer-to-peer real-time communications over a network. MMS has been used as a communication protocol for many common industrial control devices like PLCs, robots and so on (Mackiewicz 1994).

Since the TOP architecture addresses different applications (office automation etc.) from those of MAP, it is expected that some protocols also differ at the application layer. TOP also varies significantly at the physical and data link layer (real time considerations). However, many services are common for both MAP and TOP (see also Figure 2.10). 


\section{Sample Applications}

To study data flow and interaction between CIM components three sample applications were selected. The three applications are 1) a facility layout tool, 2) a simulation tool, and 3) a MRP (Material Requirement Planning) tool. This chapter explains each application's features and provides the data models needed for an integration. 


\section{Overview}

Three applications were selected to study data flow and interaction between these applications. Applications are representative of those used for planning of manufacturing layout and production. The first application, a facility layout planning software, utilizes the CRAFT algorithm to determine the best possible layout for number of departments. The second application is the simulation language SIMAN. Finally an educational version of an MRP software will be integrated with the two other applications. All three applications do not explicitly support integration or communication with other applications. Some only provide the ability to export and/or import ASCII files. None of the products utilizes a conventional database or has a database access interface.

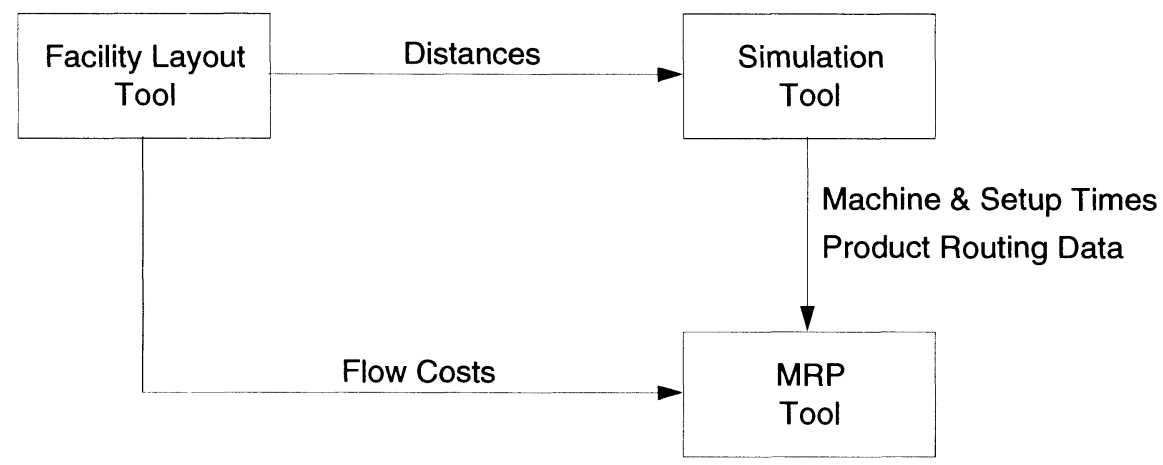

Figure 3.1 Possible Dataflow between Sample Applications.

Figure 3.1 shows some basic data flow between the selected three applications. Output data produces by one application is often used in one or even more other applications. Frequently the same data is also stored at more than one location (redundant data), which might lead to data inconsistency. The remainder of this chapter discusses each tool in terms of functionality, data model, and data 
format. The integration of the three tools will be addressed in the Integration Concept chapter.

\section{Facility Layout Tool}

QSOM (Quantitative Systems for Operations Management, Version 2.0) is a software package that consists of a facility layout module and other applications such as facility location and linear programming. This software is available for MS-DOS operating systems only. QSOM employs the CRAFT (Computerized Relative Allocation of Facilities Technique) algorithm in five different variations to solve facility layout problems.

Before a layout can be optimized, QSOM the user needs an initial layout. This can be either an existing layout or a rough approximation of the desired layout. QSOM uses quadratic cells which are identified by row and column numbers to represent departments (Figure 3.2). In addition to the initial layout, the user specifies department properties, flow between departments, and the optimization objective. The optimization objective can be either to minimize flow cost or to maximize system throughput. The user can also choose between two distance measures: rectilinear and euclidean. Flow and unit contribution between all departments are specified in a matrix. The department properties include department area, fixed, rectangular shape, and rows and columns. 


\begin{tabular}{|c|c|c|c|c|c|c|}
\hline & & & & $\mathrm{nns}$ & & \\
\hline & 1 & 2 & 3 & 4 & 5 & 6 \\
\hline 1 & 6 & 6 & 6 & $A$ & B & B \\
\hline 2 & 7 & 5 & 9 & 1 & B & B \\
\hline 3 & 7 & 5 & 1 & 1 & B & B \\
\hline 4 & 7 & 2 & 8 & 3 & 3 & 4 \\
\hline 5 & 7 & 2 & 8 & 3 & 4 & 4 \\
\hline 6 & 8 & 8 & 8 & 3 & 4 & 4 \\
\hline
\end{tabular}

Figure 3.2 Layout Representation in QSOM.

The Final Layout for Project XYZ

$\begin{array}{lllllll} & 1 & 2 & 3 & 4 & 5 & 6 \\ 1 & 6 & 6 & 6 & 9 & \mathrm{~B} & \mathrm{~B} \\ 2 & 7 & 5 & 1 & 1 & \mathrm{~B} & \mathrm{~B} \\ 3 & 7 & 5 & 1 & \mathrm{~A} & \mathrm{~B} & \mathrm{~B} \\ 4 & 7 & 2 & 8 & 8 & 8 & 4 \\ 5 & 7 & 2 & 3 & 8 & 4 & 4 \\ 6 & 3 & 3 & 3 & 8 & 4 & 4\end{array}$

Minimum value of total contribution $=1479.2$

Total iterations $=3$ by using Rectilinear distance

Total elapsed $\mathrm{CPU}$ seconds $=61.73828$

Figure 3.3 QSOM Final Layout Printout.

Once all data has been specified QSOM employs various solution methods based on CRAFT. The output is the layout in the form of an ASCII based schema and the optimized total flow cost. The final layout can be saved into a file in the same format. This is actually a disadvantage of this software since post-processing of its output is very difficult. Figure 3.3 exhibits an actual printout of the optimized layout from Figure 3.2. Department centroids and 
distances between departments of this layout have to be computed manually.

Based on the application's user-interface and file format the following IDEF1X data model can be drawn (Figure 3.4):

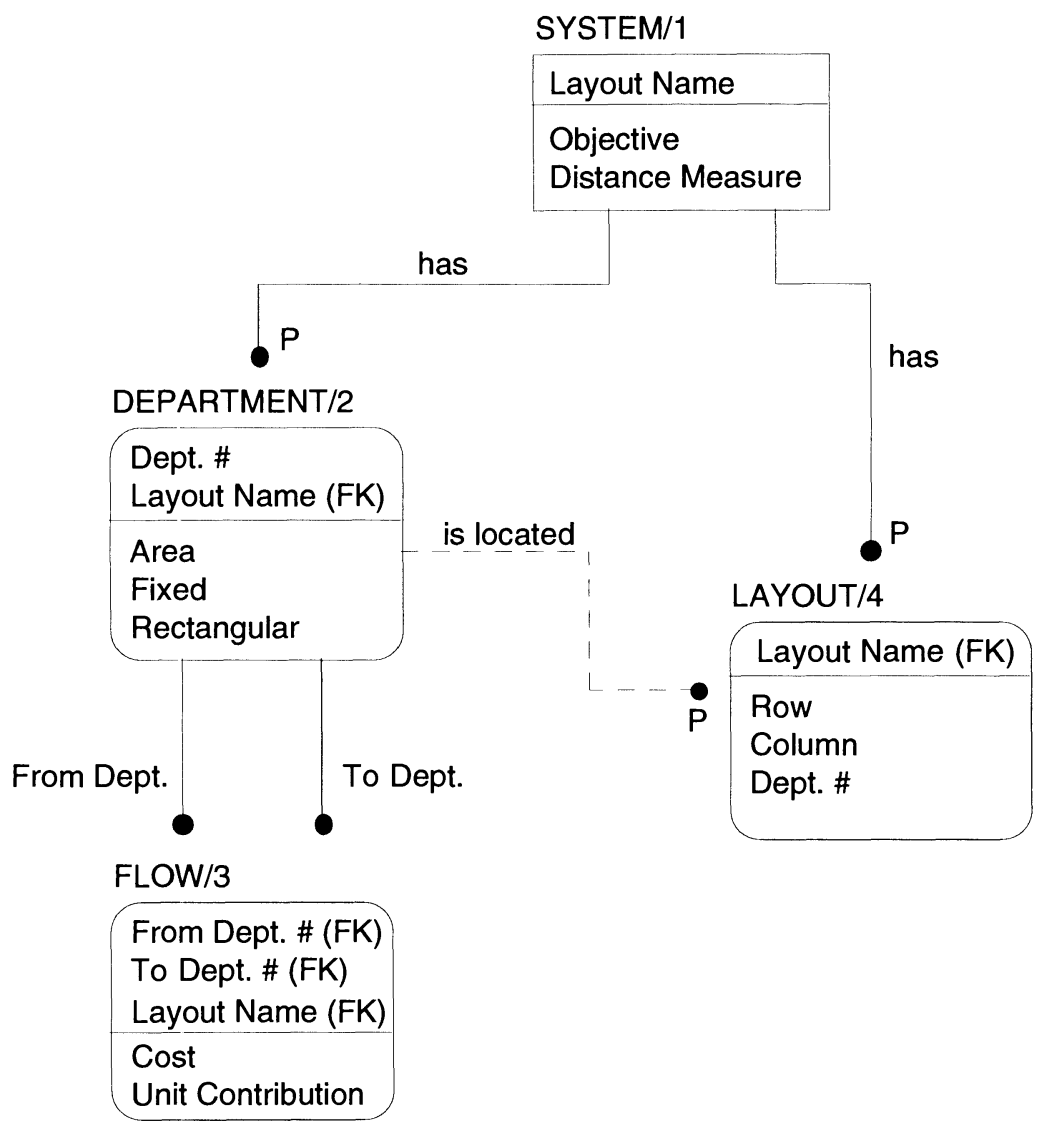

Figure 3.4 IDEF1X Data Model of QSOM's Layout Tool. 


\section{Simulation Tool}

SIMAN (by System Modeling Corporation) is a powerful and general purpose simulation language for modeling discrete, continuous, and/or combined systems. A restricted student version of SIMAN will be used. An excellent introduction to SIMAN can be found in Pedgen et al. (1990).

Systems modeled in SIMAN are segmented into a 'model' component and an 'experiment' component. The model describes the physical elements of the system such as machines, storage, transporters, and parts flow and their logical interrelationships. The experiment specifies the conditions under which the model is to run. Experiment elements include initial conditions, resource capacities, output of statistics, and the length of run time or units of production. The separation into model and experiment allows the designer to run the model with different sets of experimental data. Model and experiment are linked and executed by SIMAN to generate the response from the simulated system.

In SIMAN, the model component and experiment component are represented as ASCII text files. SIMAN recognizes model and experiment based on the filename extension ('mod or 'exp'). The first step is to compile both source files and link them into a program file that can be executed by SIMAN. Figure 3.5 shows the general procedure to simulate a system. The report file with all results of one or more simulation runs is usually stored with the project filename plus the extension 'out' (can be changed by the user). Once the model file is finished, the user can change parameters in the experiment file to run the simulation under different conditions. 


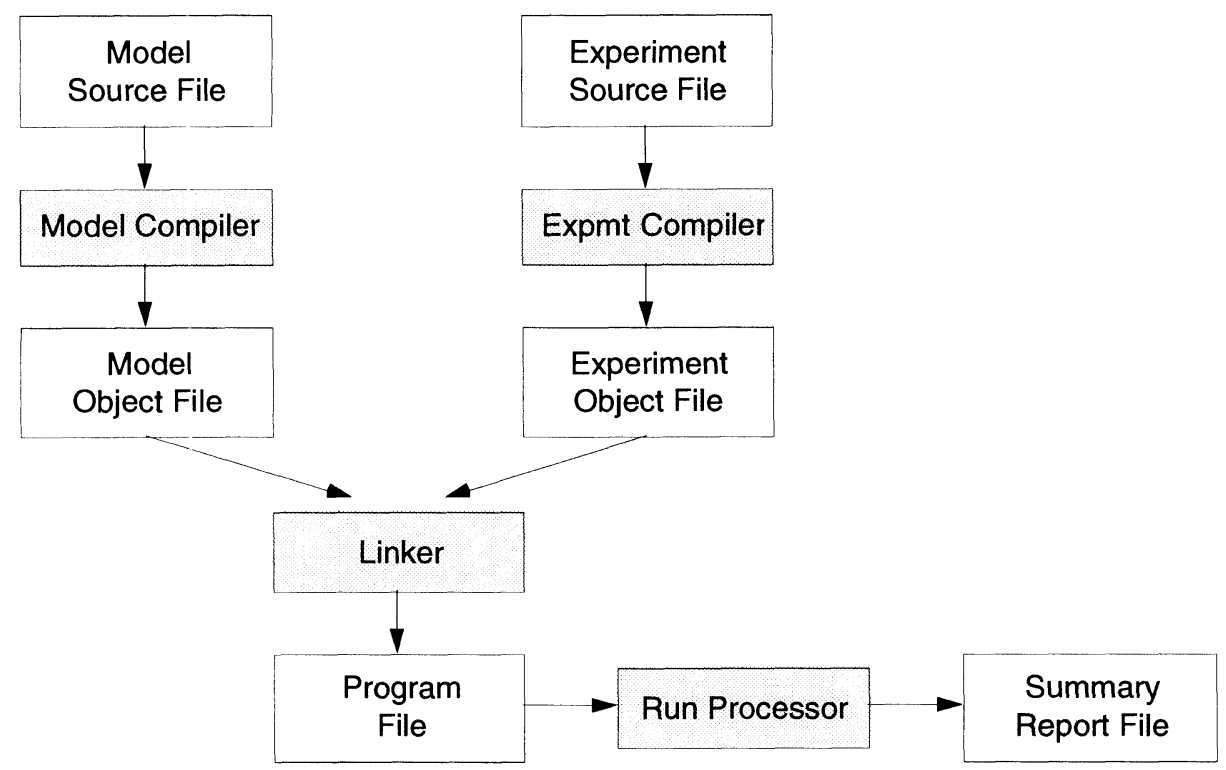

Figure 3.5 Simulation Compilation, Linking, and Execution Procedure.

Figure 3.6 contains examples of a model, experiment, and report file. The model describes a machine or workstation that processes workpieces. The machine can process one piece at a time and the process takes between 5 and 15 time units. Since the time between arrival of new workpieces is between 10 and 20 time units the machine may not be able to process a piece immediately when it arrives at the machine. Therefore, a queue needs to be in front of the machine to hold additional pieces. A counter keeps track of parts completed and the part finally leaves the system. The first keyword of every line represents a SIMAN block and can have several parameters.

The experimental file starts with a BEGIN and finishes with an END statement. The elements may be entered in any, with a few exceptions (elements that define symbolic names must precede the elements that reference those names). The 
three elements after PROJECT define what is used in the model and must therefore agree with the model file. However, element properties can be changed as desired by the user (e.g., increase queue capacity, initialize counter, etc.) to study and to optimize the system. The REPLICATE element controls simulation replications and provides length and initialization options of each replication.

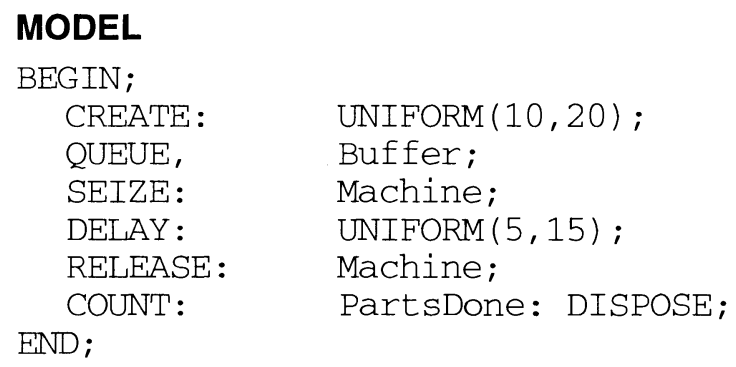

\section{EXPERIMENT}

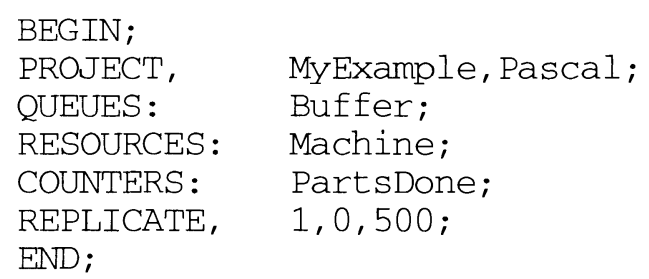

\begin{tabular}{ccc} 
Identifier & Count & Limit \\
\hline PartsDone & 20 & Infinite
\end{tabular}

Figure 3.6 Sample Model, Experiment, and Report of SIMAN.

SIMAN is a complex system with more than 50 blocks and 40 elements defined. Therefore it is difficult to develop a complete and detailed data model. 
Since the model usually does not change once finished, we are only interested in modifying the experiment file. However, the experiment contains also information (e.g., definition of queues, resources etc.) that must agree with model to work properly. Therefore, just a few elements remain that will receive their data from other applications (e.g., operations times, distances between machines, etc.).

Figure 3.7 presents a entity-relationship level (ER) IDEF1X diagram of the basic elements that will be used for this work.

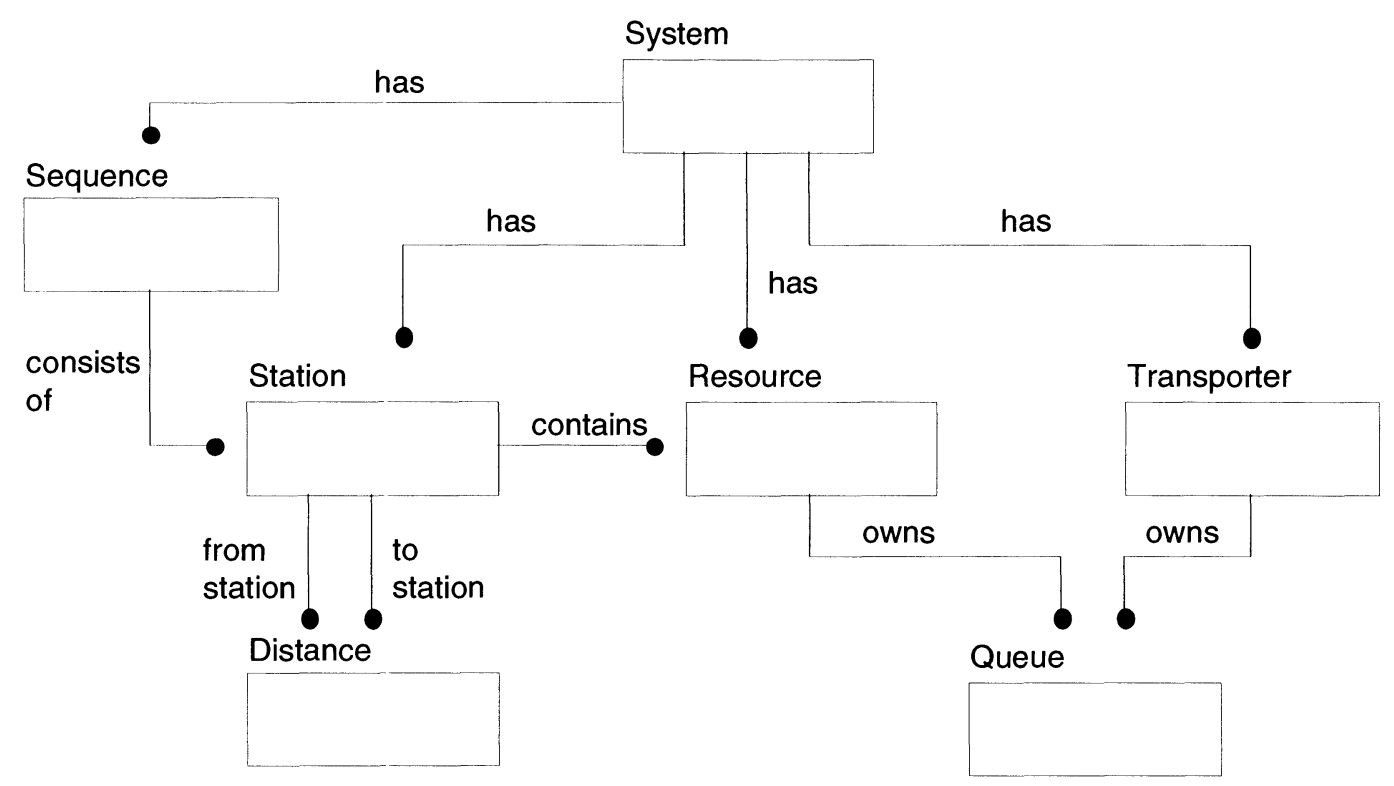

Figure 3.7 Entity-Relationship IDEF1X Data Model of SIMAN Experiment. 
Manufacturing Resource Planning Decision Support System (Chong 1989), was selected as the MRP tool. This tool is a low-cost educational MRP system. MRP-DSS includes many other systems such as automated scheduling, inventory management, and production control which are divided into several modules (Figure 3.8). It offers a text file import and export facility (Utility module) for all modules, which makes it easy to use MRP's data in other applications (gray boxes).

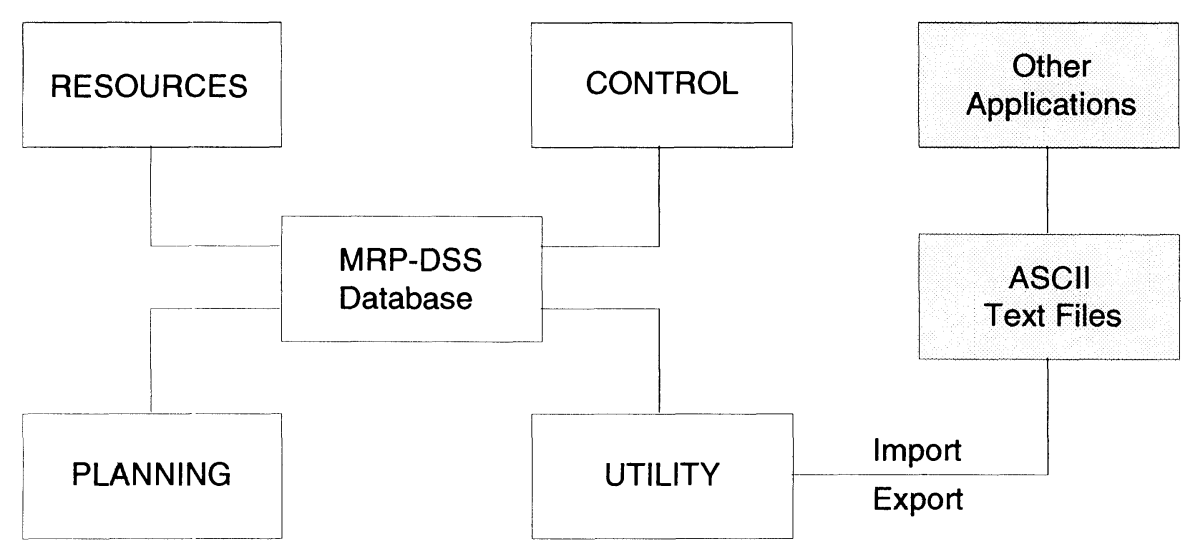

Figure 3.8 MRP Tool Block-Diagram.

In order to demonstrate an integration with the other two tools only a few modules from the resource category will be needed:

- Bill of materials data

- Machines data

- Operations and process data

- Process route data. 
Every category is exported into a different ASCII file with a fixed filename given by the MRP tool.

The bill of materials (BOM) holds a data record for every part, that specifies the part entirely (material, parent parts, components etc.). The machine data contains pertinent information about machines and stations. All operations and processes that machines can perform are listed in the operations and process data. The process route data shows the operations and processes applied to every part and therefore builds a link between part and process information.

Figure 3.9 shows an entity-relationship diagram with the relevant MRP data for this work.

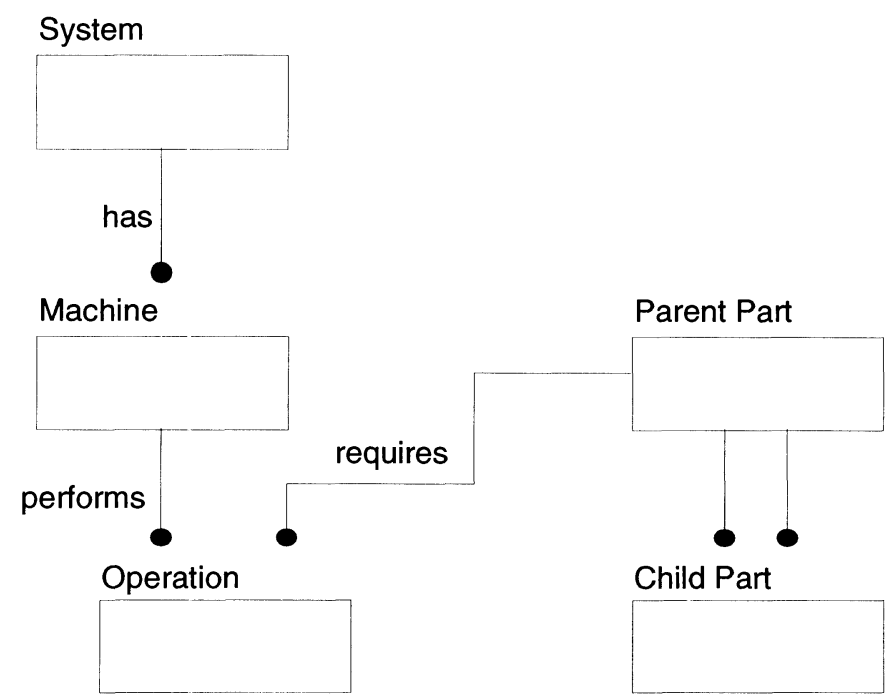

Figure 3.9 Partial Entity-Relationship IDEF1X Data Model of MRP Tool. 


\section{Integration Concept}

This chapter presents the proposed concept for integration of CIM components that are not explicitly designed for sharing their data with other components. The overview introduces the Integration Extension (IX), which acts as an intelligent bridge between CIM components. 


\section{Description}

The applications discussed in the previous chapter have common data, however, no data sharing mechanism is supplied by any application. This thesis presents a method for integration of these and other kinds of applications in a distributed environment. This integration allows applications to keep their independence (autonomy) while making some, or all, of their data available for other applications. In such a system, the user of an application does not depend on a central server system to supply the application with data.

The integration architecture consists of an intelligent extension for each application or tool in the system. This extension acts as a bridge between applications and allows them to communicate with each other. The major functions of such an extension should include:

- Network access to transport data between nodes (the application does not have to supply any network capabilities)

- Access to tool data (dependent on the application/tool)

- Translation of tool generated data into neutral format

- Data subscription mechanism with automated updating

To integrate applications and tools properly, a formal integration procedure is necessary. This procedure explains how tools and tool extensions should be configured.

Figure 4.1 shows a possible configuration with three applications to integrate. The integration extensions (IX) enable applications to communicate even 
without built-in communication functions. However, to share data, the tools have to provide some form of access to their data. Most applications use files to store input and generated data. If the file format is known it can be read and interpreted by the IX (e.g., facility layout tool and simulation tool).

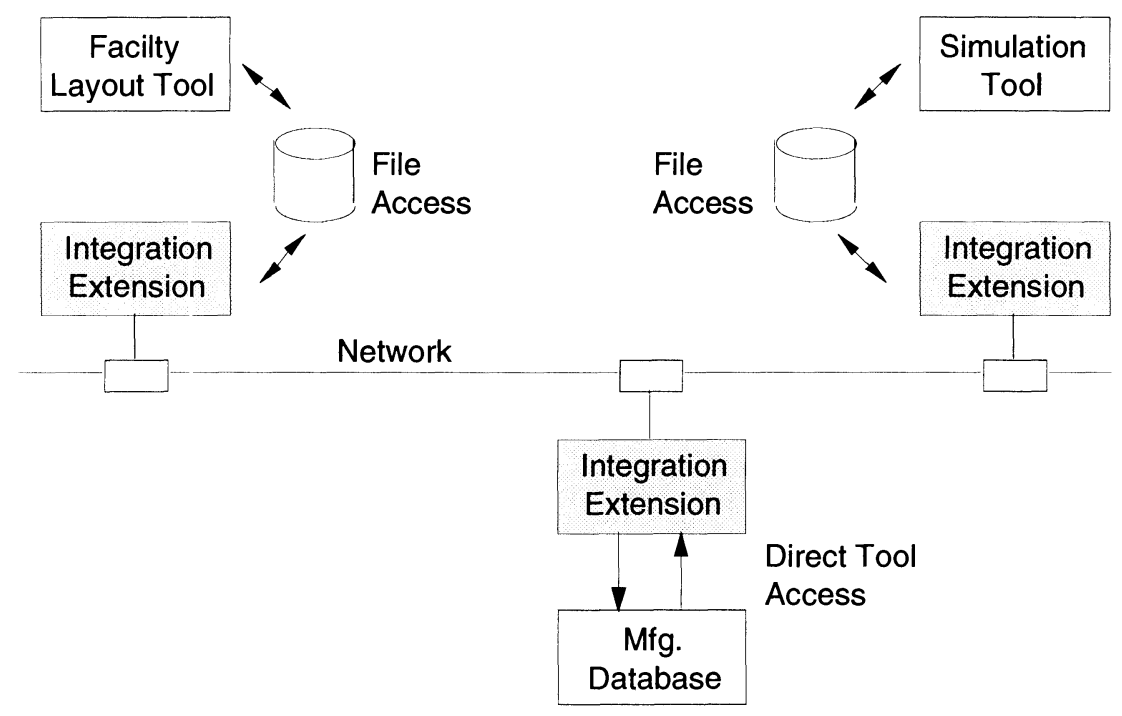

Figure 4.1 Integration of Three Applications through Integration Extensions.

Other applications may provide a more sophisticated access (direct access) through a special interface (e.g., database system). This would only require the development of an appropriate Tool Access module. For example, an SQL (Structured Query Language) Tool Access module can be developed which allows the IX to send SQL commands to any SQL-based database system. It is even possible to create access modules for shop floor equipment such as programmable logic controllers (PLC).

Once all applications are connected through individual IX, it is possible to generate an enterprise model by querying all IX for their data. Every IX carries 
a normalized data model including data in an internal database system. It is also possible to get information about shared data (common data) in the entire system.

Figure 4.2 shows the internal structure of an IX. It consists of four basic modules: Network Access Module, Link Manager, Public-Database, and Tool Access Module. The following sections explain purpose and functionality of each module in detail.

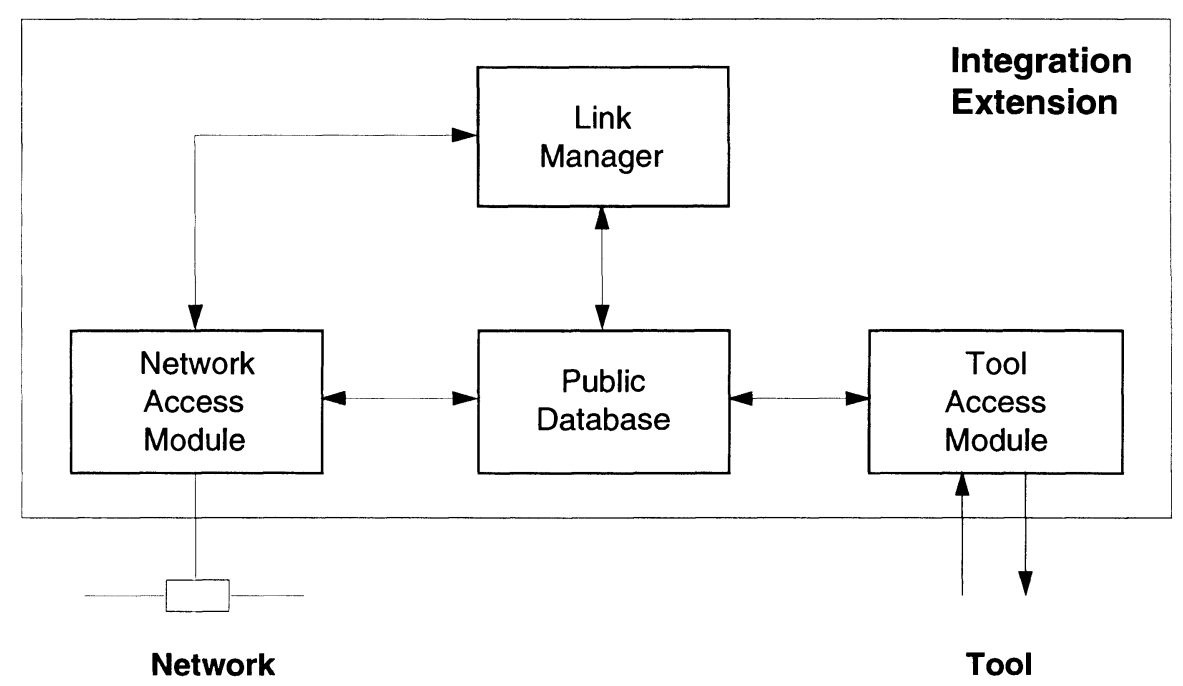

Figure 4.2 Basic Integration Extension Block-Diagram.

\section{Network Access Module}

The network access module depends on the network used. It provides a basic communication interface to open connections, send and receive data and so forth. Once a connection to another IX has been established the communication 
protocol manager in the network access module will be used to send specific command or to answer requests. Protocol managers in every IX have the same commands and answering mechanism built-in (the IX protocol). If the protocol manager receives a command from another IX it first checks it for correct syntax and then tries to answer the request. There can be only one active (sender) and one passive (receiver) connection open at the same time.

\section{Public-Database}

The heart of every IX is its Public Database (PDB). The PDB represents the normalized data model of the attached application. It contains a set of data for the application. The PDB Controller regulates the access to the PDB through a data description language and a data manipulation language. It is also equipped with additional data access and key features. The data access feature ensures that it is impossible to overwrite data produced by an application.

\section{Tool Access Module}

The Tool Access module's task is to communicate with the attached tool. As mentioned earlier, this module is customized for every application. Data will be read and written by the IX. Whenever the tool has new data available the IX will read and store it in the PDB. The translator module does the necessary translation and may even convert some of the tool's data into a neutral format. For example the output of the discussed facility layout tool provides merely a 
plan of the final layout. Therefore the translator will compute the distances between departments and store them in the PDB (according to the PDB data model). Output data from tools are usually specially marked as output data in the PDB since it wouldn't make sense to overwrite them with data from another tool. Therefore other tools can only subscribe to output data from tools. An extension in the data model will be made to address this problem.

\section{Link Manager}

The IX may also subscribe to data from other tools. The link manager maintains a small database with links to other IX. To update the tools's local database with values form other tools it has to inform the link manager. The link manager will send requests to all concerned tools and updates the PDB accordingly. If the tool's local input data or output data has changed the PDB will be updated with this data too. Once the PDB is updated it will send its content through the constructor and tool access module to the tool. The constructor makes sure that only input-relevant data is used and arranges the data in a format understandable for the tool.

\section{Sample Transactions}

An IX can perform several kinds of transactions. A transaction usually involves data transfer from one system to another system. This section demonstrates four standard transactions: store the tool's data into the PDB, store another tool's 
data (through links) into the PDB, write data from the PDB to the tool, and subscribe to data from another tool (establish links).

Update PDB (from Tool):

- Read all data from tool through Tool Access Interface

- Translate data into PDB-specific format

- Store data in PDB

Update Tool (from PDB):

- Read data from PDB according to Constructor (tool input data only)

- Send data to tool through Tool Access Interface

Update PDB (from network):

- Look up links in link database

- Connect and login to remote IX

- Retrieve desired data from remote IX

- Store data into local PDB

- Logout from remote IX

Subscribe:

- Connect and login to remote IX

- Look up data in remote IX (if necessary)

- Subscribe to desired data in remote IX

- Store subscription in remote and local IX

- Logout from remote IX 


\section{Data Model Extension}

The IDEF1X data modeling method has been used in this thesis to represent each tool's data. The development of an IDEF1X diagram is necessary to model the IX's Public-Database (PDB). However, the IDEF1X methodology is not able to completely represent data models in the PDB.

A tool normally produces output data by processing given input data. Input data as well as output data will be stored in the PDB and can be subscribed to by other tools. Every tool can also subscribe to data from other tools. However, it does not make sense to write data into the PDB fields that actually come from the tool's output data. Another similar problem arises when data in the PDB is written back to the tool. Only data designated as input data should be written back to the tool. The PDB provides a feature (data access method) to identify attributes as inputs or outputs (from the PDB's point of view).

FLOW/3

\begin{tabular}{|l|}
\hline From Dept. \# (FK) \\
To Dept. \# (FK) \\
Layout Name (FK) \\
\hline Cost \\
Unit Contribution \\
\hline
\end{tabular}
FLOW/3

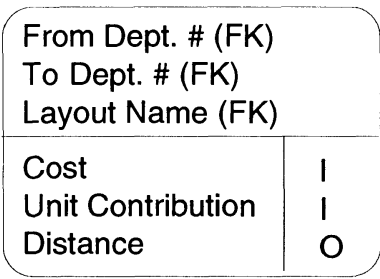

Figure 4.3 IDEF1X Extension Example.

A simple addition to the IDEF1X notation will identify the access method for every attribute. Figure 4.3 shows an entity from the facility layout data model. 
The left FLOW entity describes exactly how the flow data is entered into the facility layout application. However, the output produced by the application needs to be stored in the IX data model too. The output is for this example the distances between the departments (derived from the final layout). Therefore, the distance attribute is an output-attribute that can only be changed by the facility layout application.

\section{Integration Procedure}

The integration procedure covers all steps necessary to hook up an application to an IX. The general procedure includes the following steps:

- Establish normalized data models of tools data with IDEF1X

- Specify PDB with the IX data description language

- Customize tool access interface, translation, and construction modules

The first step is to analyze the tool's data. Usually this is done by studying the input and output files. However, it is not always easy to do, since some tools use proprietary file formats. Once the tool's data is known, a normalized data model can be established by using a data modeling technique, such as IDEF1X.

The data model will be represented by the IX's data description language in the PDB. Finally the tool access module has to be customized to store and retrieve data in the PDB. This is can be the most complicated and time-consuming step. The translator must read the tool's data and to store it in the PDB. Usually the translator takes individual data instances and inserts them into the correct table. 
However, the translator also has to convert and store data that is not explicitly stored by the tool. The constructor does the opposite of the translator.

\section{Framework Implementation}

A major part of this thesis is the implementation of the Integration Extensions for the three selected sample applications. All three applications require MSDOS operating system version 3.3 or higher.

The requirements for the development system of the Integration Extension should include a simple network access interface, easy to design user interface, elementary file input/output functions, the possibility to develop and integrate functions that are not already provided, and short development time. HyperCard (from Apple Computer) is a development system that meets the above requirements. Since HyperCard runs on Macintosh computers the sample applications will be executed on the SoftPC (MS-DOS) emulator. The HyperCard environment will be discussed more in detail in the Implementation chapter. 


\section{Software Design}

This chapter explains the software design of the Integration Extension. It describes all modules (Network Access, Public-Database, Tool Access, and Link Manager) and their functionality. 


\section{Design Approach}

The IX design approach is based on DFDs (data flow diagrams). DFDs show data and control flow between functional entities. A data flow design starts out with a level 0 DFD to identify the borders and interfaces of the system.

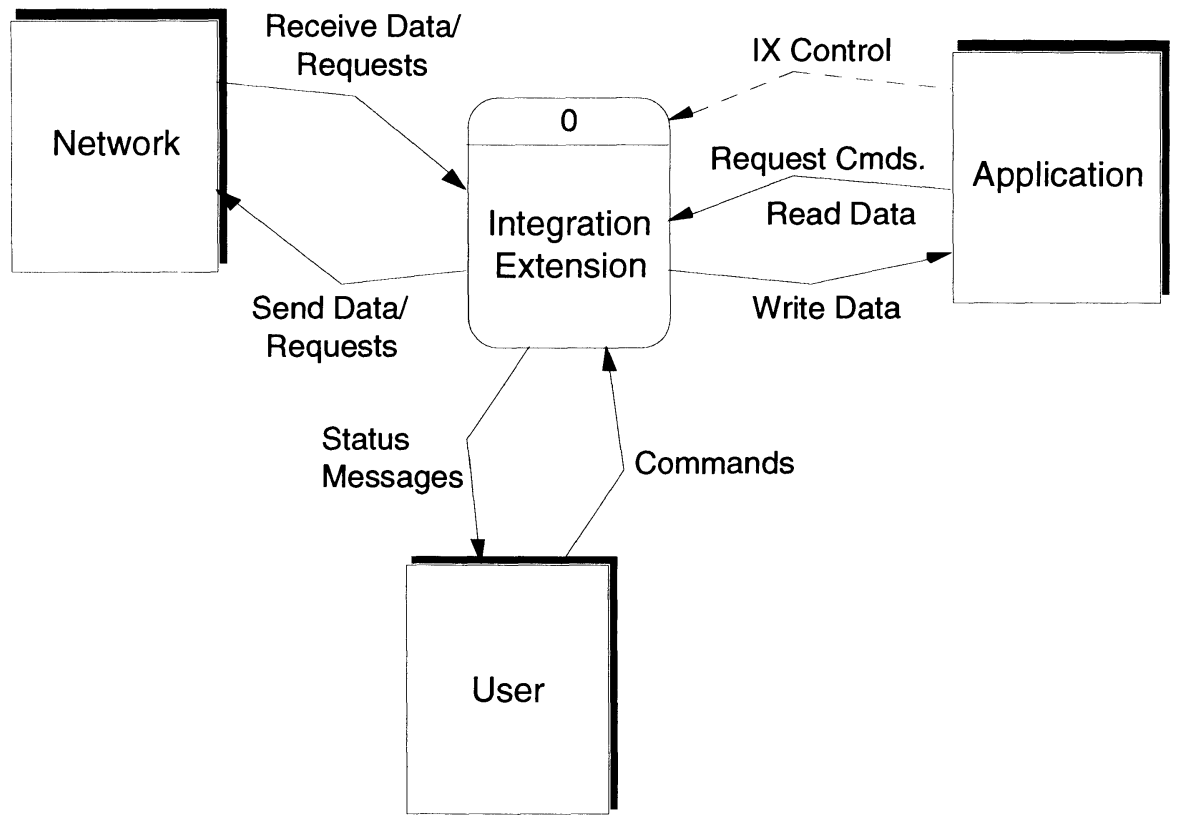

Figure 5.1 Level 0 Data Flow Diagram (DFD0).

An IX interacts with three external entities (Figure 5.1). All three entities send and/or receive data to/from an IX. Once the IX is completely configured the user entity is only used to monitor the IX.

Figure 5.2 shows the decomposition of the DFD level 0. It consists of eight functional units and three data storage. 


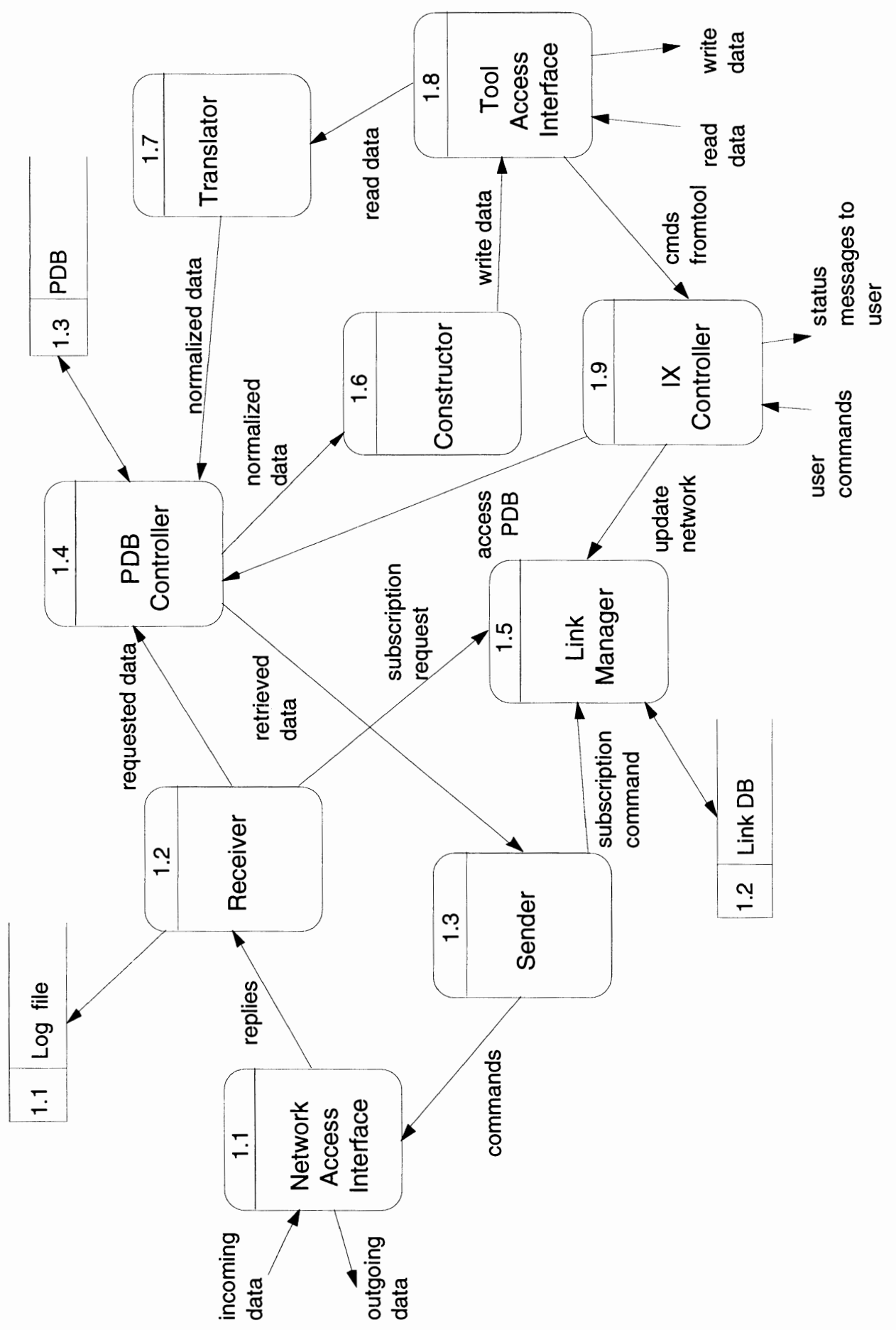

Figure 5.2 Level 1 DFD Shows Data Flow between Main IX Modules. 


\section{Functions:}

- Network Access Interface (1.1)

Provides network dependent access functions. Maintains two logically independent communication channels.

- Receiver (1.2)

Sorts out incoming messages and forwards them to the appropriate module. Writes all logins into an access-log file.

- Sender (1.3)

Ensures correct format of outgoing messages (syntax).

- PDB-Controller (1.4)

The PDB controller supports an SQL-like data-definition and datamanipulation language. Only this module can read and write from/to the PDB.

- Link-Manager (1.5)

Keeps records of all links from and to this IX.

- Constructor (1.6)

Converts normalized data from the PDB into a tool specific format.

Constructors are customized for each tool.

- Translator (1.7)

Converts data from the attached tool into normalized data (as defined in the PDB). Translators may store irrelevant data into a separate storage, that can be retrieved by the Constructor.

- Tool Access Interface (1.8)

Provides tool dependent access functions to read and write data. 
- IX Controller (1.9)

Allows IX access through user-interface.

\section{Storages:}

- Log file (1.1)

Contains a record for every login and logout through the network. Every entry includes date, time, and login name.

- Link DB (1.2)

Holds records for every link to or from this IX. Every link entry has an unique identification number (within IX).

- Public DB (1.3)

Stores all relevant data from the tool in a normalized format by using tables (entities) and predefined attributes for numbers and text. 
This section covers the four basic modules: communication, internal database, tool access, and link manager.

\section{Communication}

The communication module consists of two parts: the network access part and the protocol manager. The network access module is network protocol dependent and works only with the network for which it is built. The implementation for this work is developed for TCP/IP network access. Once a connection between two systems has been established it is necessary that both systems speak the 'same' language. This language (or protocol) is not platform or system dependent. Figure 5.3 shows the network model used with ISO-OSI reference model terminology.

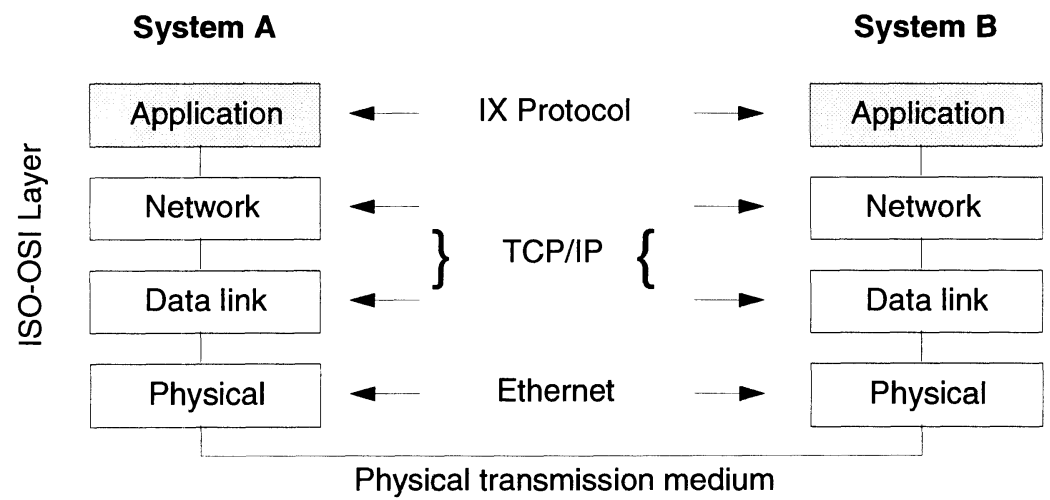

Figure 5.3 IX Network Layer Model. 
Layers one to three represent widely used standards and protocols that will also be used for this thesis. Ethernet (IEEE standard 802.3) is readily available and fast enough for this application (10 Mbits/sec). TCP/IP for layer two and three was chosen for many reasons. As an open network protocol standard TCP/IP is available on virtually every platform. This makes it easier to extend IX's to other platforms. TCP/IP protocols are also easy to implement. The application layer defines the IX communication protocol (IX-P) and therefore enables individual IX to communicate with each other in a protocol language. No other layers from the ISO-OSI reference model (page 38) are used.

\section{Network Access Interface}

Although the network access in this thesis will be realized by using the TCP/IP protocol, other network protocols maybe used instead. Therefore the network access defines just a few interface functions that have to be implemented for the chosen network. Other modules, namely the IX-P manager, can then use these functions without knowing the type of network on which they are communicating. Table 5.1 lists the required functions of the network access module.

Table 5.1 Network Access Interface NetworkInit Initializes network resources. In: Out: -

NetworkDispose Releases network resources and terminates all connections.

In: -

Out: - 


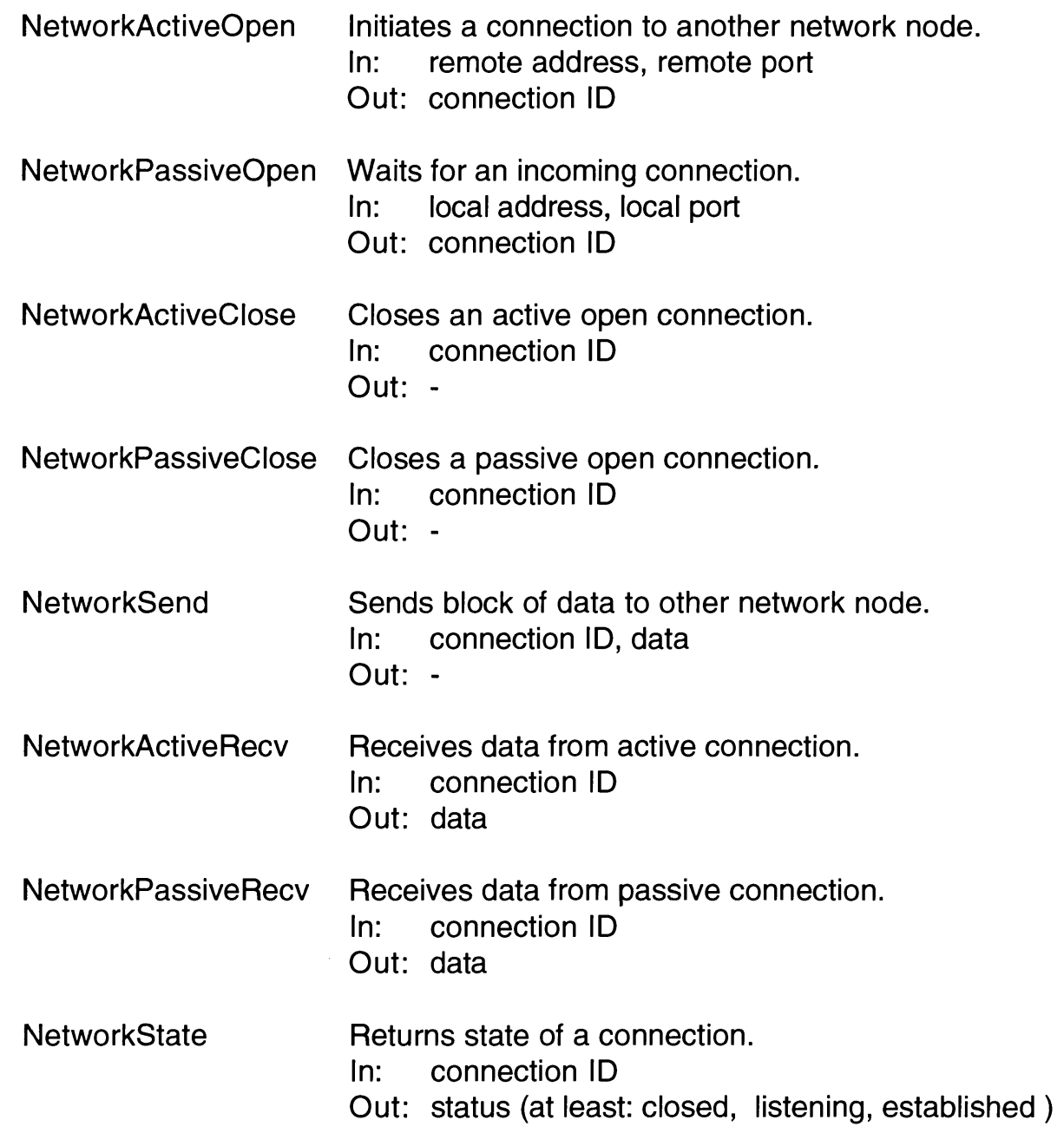

The network access module does not support node registration in this design. Dynamic registration of nodes in a distributed network is more difficult since there is no global authority that keeps track of all network nodes. A possible solution of dynamic node registration is realized in AppleTalk's ALAP (AppleTalk Link Access Protocol) and NBP (Name Binding Protocol). However, this implementation requires the user to enter other node address in a provided list. 


\section{Communication Protocol}

The IX communication protocol (IX-P) defines a set of commands and related reply codes. Since an IX can have an active and a passive connection open at the same time it acts as sender and receiver. Therefore the IX-P can be divided into sender part and receiver part (Figure 5.4). Every IX must provide the basic set of the IX-P.

IX Node A

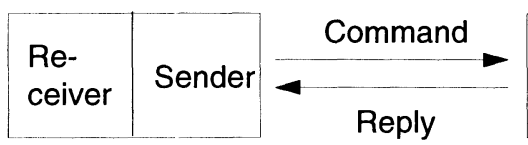

IX Node B

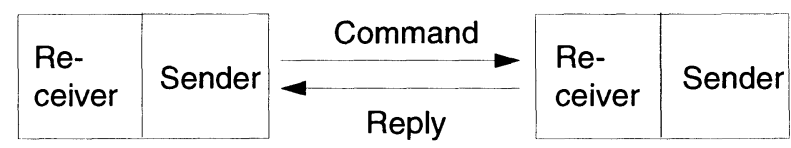

Figure 5.4 IX Sender and Receiver Communication.

A connection is always initiated from a sender while receiver is passive and waits for a connection. There can be maximum one active and one passive connection at the same time. The data flow is controlled by the sender that issues a command to the receiver. The receiver answers a command and then waits for another command. A receiver does never send data without a request. Figure 5.5 shows how the IX receiver processes incoming data in a state-event diagram. Once a passive connection has been opened (usually at IX startup time) the receiver is in the NC (not connected) mode. If another node connects to an IX in the NC mode, the receiver will reply with a greetings message and goes into the NDM (normal disconnected mode). After successful identification of the sender the receiver will finally move into the NRM (normal response mode) to process incoming commands. The receiver goes back to the NC mode immediately after termination of the connection by the sender and listens for a 
new connection.

Every connection and disconnection will be registered and written to an ASCIItext log file. A log entry includes date, time and the login-name of the sender. There is no password necessary to login to an IX, however, and an additional password could be built into the NDM easily.

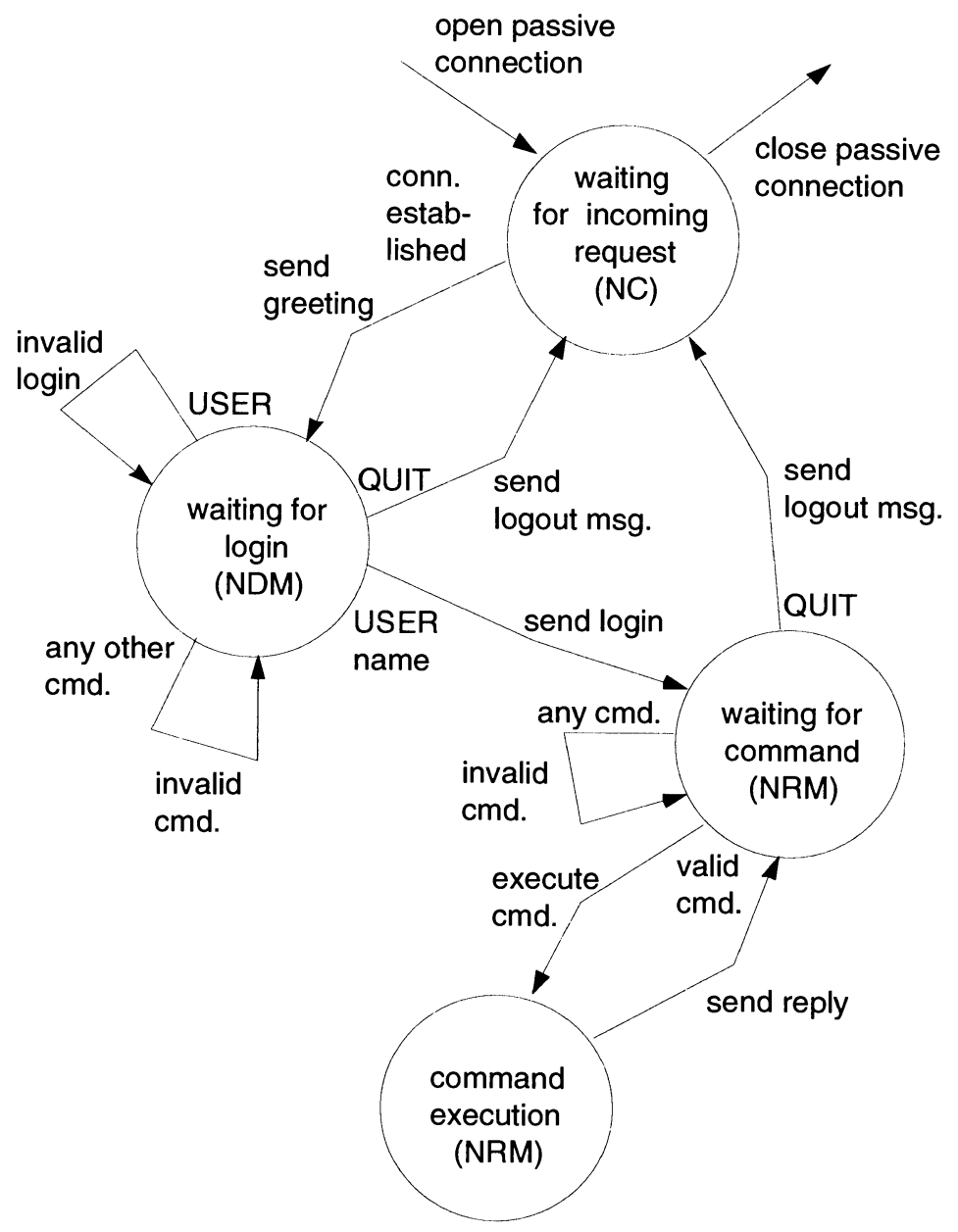

Figure 5.5 IX Receiver State-Event Diagram.

The IX protocol defines commands and reply codes for communication between 
two nodes. Commands can only be used by sender IX's while reply codes are only used by receiver's. The IX protocol is an ASCII-based protocol similar to FTP (file transfer protocol) or SMTP (simple mail transfer protocol). IX commands (Table 5.2) consist always of a four-letter keyword followed by parameters, if applicable.

Table 5.2 IX Protocol Commands

USER username Allows user or system to login to the IX. Reply codes: 110, 111

QUIT

Quits the open connection.

Reply code: 120

LIST $^{\star} \quad$ [table]

Lists all tables available in this IX. If a table name is specified it return a complete list of attributes for this table.

Reply codes: 200,220

QUER $^{*} \quad$ table attribute value [attribute_n] [value_n] Retrieves requested data from IX.

Reply codes: 200, 221, 222, 223, 224

SUBS* localLinkID table attribute keys Subscribes to specified data and returns the remote link ID. Reply codes: $200,240,243$

UNSB* $^{*}$ remoteLinkID Removes specified subscription.

Reply codes: 200, 240, 242

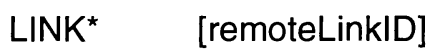

Shows all links and link IDs to other systems of this IX.

Reply codes: $200,240,241$

NTFY* remoteLinkID status

Notification regrading data that becomes unavailable (when local data will be removed)

Reply codes: $200,240,242$

SYST $^{\star} \quad-$

Returns the operations system and version number .

Reply code: 200 


\section{HELP* -}

Shows all available commands with parameters.

Commands with an asterisk $\left(^{*}\right)$ can only be used in NRM.

Fields in brackets [] are optional.

Command parameters are separated by spaces (ASCII character 32) and terminated by return (ASCII character 13). An example of a valid command that retrieves all data from the table 'Department' with the attribute 'Area' equal to 5 would be:

\section{QUER Department Area 5}

An answer will be sent back to any incoming command (whether a command has been executed successfully or not). All answers start with a reply code followed by the data requested or other information. Table 5.3 lists all possible reply codes sorted by mode. The explanatory text after every reply code helps understanding the reply code when the sender is an user-controlled application (IX access trough terminal emulation e.g., telnet). An IX will usually ignore any text after the reply code on first line.

Table 5.3 IX Protocol Reply Codes

Normal Disconnection Mode (NDM)

100 Greeting when connection has established, ask for user ID

$110 \quad$ User login accepted

111 User login invalid. Access denied.

112 Invalid command in NDM

120 Logout message 


$\begin{array}{ll}\text { Normal } & \text { Response Mode (NRM) } \\ 200 & \text { Command OK } \\ 210 & \text { Command syntax error } \\ 211 & \text { Invalid command in NRM } \\ 212 & \text { Command not implemented } \\ 213 & \text { Internal error in remote unit } \\ 220 & \text { Table does not exist } \\ 221 & \text { Attribute does not exist } \\ 222 & \text { Record does not exist } \\ 223 & \text { No value available } \\ 224 & \text { Data not found } \\ 240 & \text { Internal link error in remote unit } \\ 241 & \text { No links available } \\ 242 & \text { Link does not exist } \\ 243 & \text { Link already exists }\end{array}$

Figure 5.6 demonstrates a possible IX communication session. The sender asks for a complete list of tables in the remote IX followed by a detailed tables request. 
Sender IX

Open Active Connection

USER Simulation

LIST

LIST Apple

LIST System

QUIT

Close Active Connection

\section{Receiver IX}

100 IX Receiver 1.0 ready.

110 User Simulation logged in

200 Command OK.

TABLE System

TABLE Department

TABLE Flow

TABLE Location

220 Table does not exist

200 Command OK

TABLE System

LayoutName CHAR40 I P

Ojective INT I

$4 \quad 120$ User Simulation logged out

Figure 5.6 Example IX Communication Session. 


\section{Internal Public-Database}

The internal database provides storage for the PDB. This section explains the database model and access routines that are used by the PDB. The PDB consists of one 'structure file' plus data files for every table used.

The structure file simply holds a list of all tables created. Therefore the structure file acts as a directory to what is available in the IX. It is an ASCII textfile that has the following format:

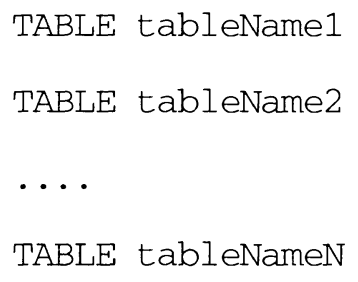

The structure file will be updated when creating a new tables or removing an existing table. There can be only one structure file in an IX.

The table files contain the actual data preceded by a well defined header. All attribute definitions of a table are stored in this header. An attribute is defined as follows:

- Name

- Type

- Access

- Key

An attribute name can have up to 19 characters. The attribute type must be one of the following: INT ( 2 byte integer), REAL ( 8 byte floating point), 
BOOLEAN (1 byte, valid values: T or F), and CHARxxx (character string) where $\mathrm{xxx}$ represents the length of the string (maximal 999 characters). The access field ( 2 bytes) regulates access privileges of attributes. Currently there are two valid entries defined for this field: I and O. An attribute defined as 'I' can subscribe its content from another IX. If the IX is not allowed to write into this attribute is must be set to ' $O$ '. However, other IX may subscribe to any 'I' or ' $\mathrm{O}$ ' attributes. The key field identifies attributes as key attributes. It can contain P (primary key), F (foreign key), or nothing.

\section{Database Access Interface}

The database access interface for the PDB consists of the data description language (DDL) and the data manipulation language (DML). The DDL provides functions for the user to create, delete, and retrieve tables and to add attributes to tables (Table 5.4). Some functions returns a status message ( 2 byte integer) which usually indicates success or error type after executing the function (Table 5.6)

Table 5.4 Data Description Language

CreateTable Adds new table definition to the structure file and creates the actual table file. It creates also the structure file if it does not already exist.

In: structureFile tableName

Result: status *

AddAttr Adds an attribute to the table.

In: tableName attributeName attributeType access key

Result: status *

ShowTable Lists all attributes (name, type, access, keys) of a table.

In: tableName 
Result: empty string or table attributes

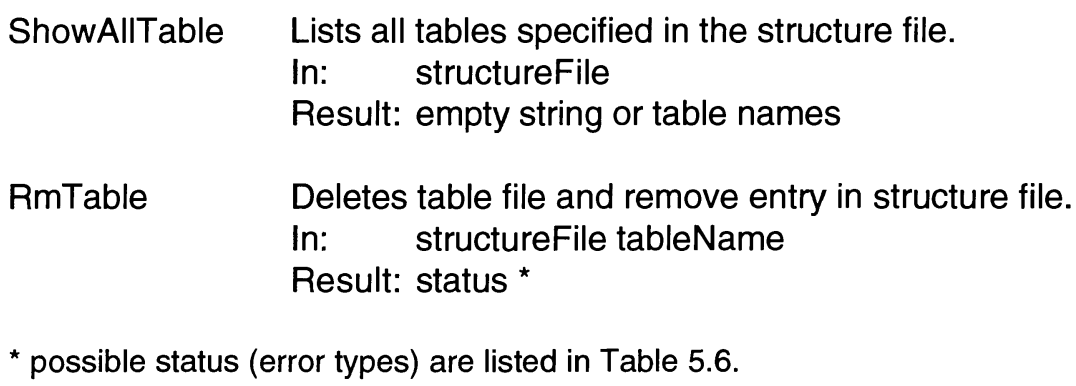

Table 5.5 Data Manipulation Language

AddRec

Adds a new record to the table.

In: tableName data

Result: status *

RmRec Removes existing record from the table.

In: tableName recordNo

Result: status *

SearchRec Searches for records that match given attribute and value

In: tableName attribute value

Result: recordNo or 0 if not found

SearchAndRec Searches for records that match all given attributes and values (AND combination)

In: tableName attribute1 value2 [attribute2 value2]

Result: recordNo or 0 if not found

GetRec Retrieves recordNo from table

In: tableName recordNo

Result: recordNo or 0 if recordNo does not exist

SetRecValue Changes value of attribute of recordNo

In: tableName attribute value recordNo

Result: status *

GetRecValue Retrieves value from attribute of recordNo

In: tableName attribute recordNo

Result: empty string or requested value

UpdtTableVal Updates a specified value with given key values

In: tableName keyAttr1 keyValue1 attr value

Result: status *

UdptTableRec Updates entire record with given key values

In: tableName keyAttr1 keyValue1 attr record

Result: status * 
NumOfRecords Counts number of records in table

In: tableName

Result: 0 or number of records

* possible status (error types) are listed in Table 5.6.

The DML (Table 5.5) provides a variety of functions to store and retrieve data from the PDB. Data fields within records are delimited by a tab character (ASCII character 9).

DDL as well as DML functions always return a result. The result can be either an error message or the requested data depending on the function. Table 5.6 shows all possible error numbers. Error numbers are always negative to distinguish them from real data. Real data includes both records numbers (always positive or zero) and data retrieved from the database.

Table 5.6 List of Possible DDL and DML Errors

$\begin{array}{lrl}\text { noErr } & 0 & \text { No error } \\ \text { kFileErr } & -1 & \text { File l/O error } \\ \text { kFileNotFnd } & -2 & \text { Cannot find or open file } \\ \text { kArgsErr } & -3 & \text { Wrong number of arguments } \\ \text { kTableErr } & -10 & \text { Table does not exist } \\ \text { kRecErr } & -11 & \text { Record number does not exist } \\ \text { kAttrErr } & -12 & \text { Attribute does not exist } \\ \text { kValErr } & -13 & \text { Value does not exist } \\ \text { kAccErr } & -14 & \text { No read access of this attribute } \\ \text { kKeyErr } & -15 & \text { Attribute is not a key attribute } \\ \text { kATypeErr } & -16 & \text { Invalid attribute type } \\ \text { kNotFound } & -20 & \text { Cannot find value }\end{array}$




\section{Tool Access}

The Tool Access module consists of three submodules: Tool Access Interface, Translator, and Constructor. Although all three submodules are dependent on the attached tool and its data, the submodules have tool independent access interfaces. Figure 5.7 shows the collaboration among the Tool Access modules.

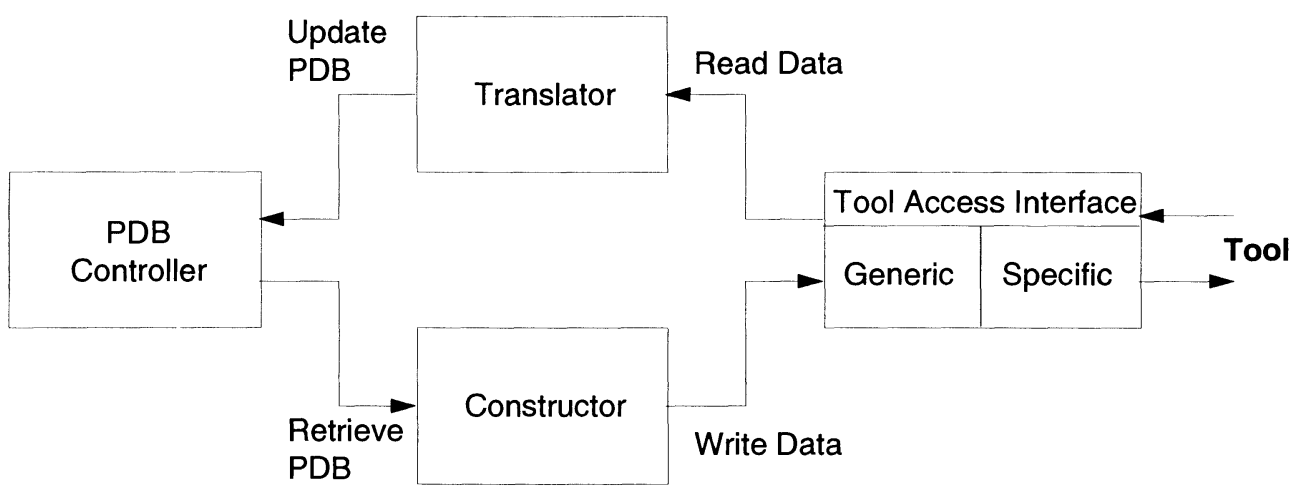

Figure 5.7 Tool Access Module Components.

This Tool Access design does not support any translation tables. The use of translation tables would make it easier to write translators and constructors.

\section{Tool Access Interface}

The Tool Access Interface provides the 'physical' access to the tool through an interface of three functions: ReadToolData, WriteToolData, and ToolControl. ReadToolData and WriteToolData reads/write any kind of data from/to the tool. 
Usually this is done by reading or writing data files. In this case ReadToolData merely returns a pointer to the data read. Similarly data is written to a file just providing the pointer to the data. However, the Tool Access Interface could use other ways to communicate with tools. For example, the Tool Access Interface could send and receive data through a serial port.

The ToolControl function allows tools to send commands to the IX without using the IX's user interface. The following three commands have been predefined:

- Update PDB from Tool (\#1)

- Update Tool from PDB (\#2)

- Update PDB from Link Table (\#3)

Other commands may be added. In order to use this feature the tool must have the ability to call the ToolControl function.

Translator

The Translator processes the incoming data into a 'normalized' format which is suitable for the PDB. Thus the Translator goes through the data read by the Tool Access Interface and updates the PDB. The update function provided by the PDB module (UpdtTableRec) searches the record in PDB to be updated by using the key attributes. The existing data in the PDB will be overwritten with the new data. If no record exits, a record with the new data will be added to the appropriate table (AddRec). 


\section{Constructor}

The Constructor does the opposite of the Translator. It reads data from the PDB and puts it into a format understandable by the tool. Like the Translator, the Constructor must have the exact knowledge of the tool's data format. The order of data written to the tools is crucial and specified in the Constructor module itself.

\section{Miscellaneous Tool Data}

Some tool data may not be used or needed in the PDB and is therefore not translated into the PDB. This means that the PDB lacks some of the tool's data. A problem will arise when the contents of the PDB is written back to the tool. Obviously the tool will miss this data and may not be able to use the data properly again.

A simple mechanism takes care of this problem by writing all unused tool data into a separate file during translation time. When writing the data back to the tool, the constructor takes the additional data from this file and the PDB and builds the final tool data file. Since there can be multiple section in the tool data file that go into the separate data file, it becomes necessary to put a special tag in front of every section. This mechanism assumes that the tool data is kept in the same internal sequence. 


\section{Link Manager}

The Link Manager keeps track of links to other IX components. For this purpose the Link Manager maintains a small database. This database does not store any data from the PDB. An entry in the link database occurs with every subscription command. Both source and target will register the subscription in their personal link database. This makes it possible for publisher of data to notify subscribers in case of unavailable data on the publisher's side.

The link database format defines the following fields for every link:

- Link ID

- Remote node address

- Remote link ID

- Link type (S or P)

- Link status (A, T, D, N, F, or S)

- Table, attribute, keys

The link ID is a number (integer) that is unique for that IX. The IX assigns numbers consecutively (starting with 1 ) for every subscription. The remote node address is an character string with the remote's IX node address (depends on the network used). Link ID and node address together identify links when accessed from other IX's. The link type specifies whether the local IX is publisher $(\mathrm{P})$ or subscriber $(\mathrm{S})$ of the data specified. Link status maintains information about the current availability of the linked data and is dependent on whether the particular IX is publisher or subscriber of the data. The following list shows possible status values for publisher and subscriber: 


\section{Publisher}
A Link available
$\mathrm{T}$ Link temporarily unavailable
D Link deleted

\section{Subscriber}

S last update successful

F last update failed

$\mathrm{N}$ link never updated

The remaining fields identify table, $\operatorname{key}(\mathrm{s})$, and attribute of the local PDB. These entries depend also whether it is a publisher or subscriber IX. A publisher IX lists data that it makes available to others, while a subscriber records where the subscribed data will be stored in the local PDB. All fields are delimited by tab characters (ASCII character 9) and terminated by carriage return + linefeed (ASCII characters 13 10). The IX-P has already defined four commands to manage links over a network: SUBS, UNSB, LINK, and NTFY.

\section{Publisher IX}

Node: Layout

\begin{tabular}{l|l|c|c|c|l|c|l} 
ID & R-Addr & R-ID & Type & Status & Table & Attr & Key1 \& Value1 \\
\hline 1 & Simu & 5 & P & A & System & Rows & LayoutName = Term Project \\
2 & $\ldots \ldots$ & & & & & &
\end{tabular}
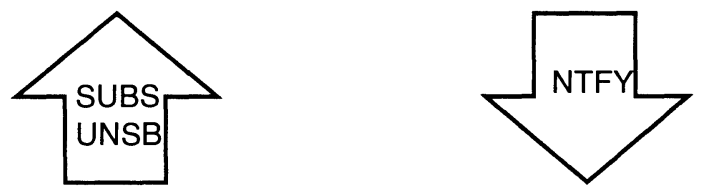

\section{Subscriber IX}

Node: Simu

\begin{tabular}{l|l|c|c|c|l|l|l} 
ID & R-Addr & R-ID & Type & Status & Table & Attr & Key1 \& Value1 \\
\hline 1 & $\ldots \ldots$ & & & & & & \\
5 & Layout & 1 & S & N & System & Rows & LayoutName = Term Project
\end{tabular}

Figure 5.8 Link Database Example Entries. 
Figure 5.8 gives some example for entries in publisher and subscriber link databases and commands that will be used for the transactions. The publisher is a layout application. Another simulation application IX wants to subscribe to a certain value in a table.

A successful subscription command (SUBS) creates an entry in both IX link databases. The subscriber status will be set to ' $N$ '. Usually the subscriber tries to update its link by executing a query to the specified data. The data received from the query will be stored in the PDB. If the subscriber does not need a remote's IX data anymore, it sends an UNSB command to the appropriate IX. The links in both IX's will be removed. However, if a publisher no longer wishes to publish data that has been subscribed to by other IX's, it will send an NTFY command to all concerned IX's. The publisher will keep the link in its database (status set to 'D') until all IX's have confirmed the NTFY command. 


\section{Implementation}

This chapter discusses the implementation of the IX by using the HyperCard development environment. A brief introduction into Hypercard and its external functions capability is followed by a description of the actual user interface of the IX. 


\section{Development Environment}

The HyperCard software construction tool was selected to implement Integration Extensions. The benefits of HyperCard have already been mentioned in the Concept Chapter (page 65).

Information in HyperCard appears on cards. Cards are grouped together into stacks. Therefore, an IX is a stack with different cards that contain different viewpoints of an IX. Cards can consist of buttons, fields, backgrounds, icons etc. Buttons usually perform tasks, such as store data, go to another card etc., if pressed by the user. Fields are used to entered or to display any kind of information (text, images etc.).

Normally a stack runs in the HyperCard application, however, it is possible to create a standalone application that does not depend on the HyperCard application. See (HyperCard Reference Manual 1993) for more information.

\section{HyperTalk}

The scripting language built into HyperCard is HyperTalk, that is English-like and very easy to use. HyperTalk is used to send messages to and from HyperCard objects such as buttons, fields, cards, windows, and stacks. How an object responds to a particular messages depends on the object's script. The script to put some text into a field could look as follows:

$$
\text { put "This is an alert" into field "alert" }
$$


HyperTalk provides dozens of commands and functions such as file I/O, mathematic functions, drawing, and so on.

\section{External Commands}

If a command or function does not exist in the HyperTalk vocabulary, HyperCard provides an elegant way to write external commands (XCMD) and external functions (XFCN). External commands (and functions) are executable Macintosh code resources, written in a Macintosh programming language (such as Pascal or C), which are attached to the HyperCard stack. Once an XCMDs or XFCNs has been attached to a stack, it a can be called from HyperTalk in the same way that built-in commands or user-defined message and function handlers are called. (HyperTalk Script Manual 1993) contains all details about the development of XCMDs and XFCNs.

For this implementation, external commands and functions are used in the Network Access Interface and the internal Public-Database. The Network Access Interface XCMDs come from the Apple HyperCard MacTCP Development Toolkit. This toolkit includes 13 XCMDs to access networks through TCP. The PDB XCMDs were developed as a part of this thesis. They are written in the $\mathrm{C}$ language with the Metrowerks CodeWarrior for Macintosh development environment. 


\section{User Interface}

Every IX HyperCard stack has a main card that includes all major elements to control a configured IX. The main card is always the first card shown when an IX is launched. There are several other cards accessible from the main card to configure and monitor the IX. Figure 6.1 shows the typical IX user interface structure.

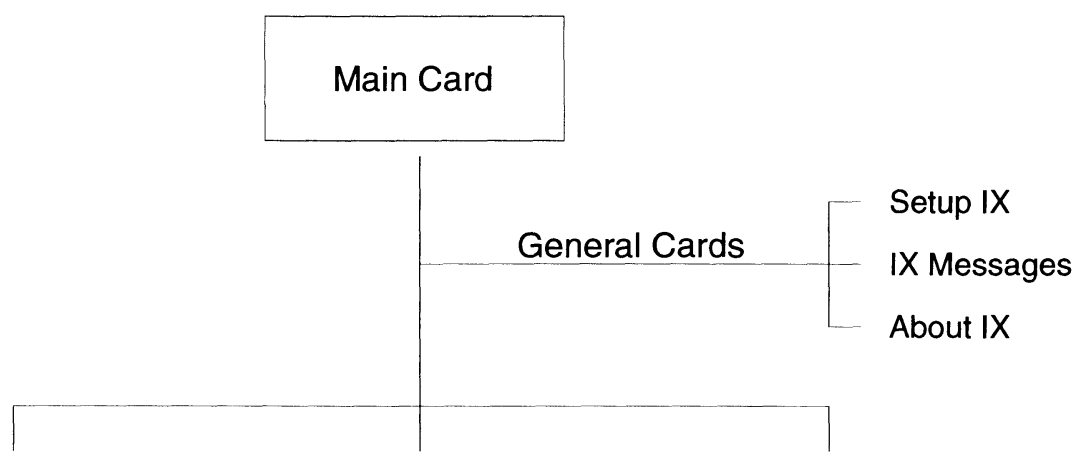

Network Access Cards

Network Setup

Access Log
PDB Cards

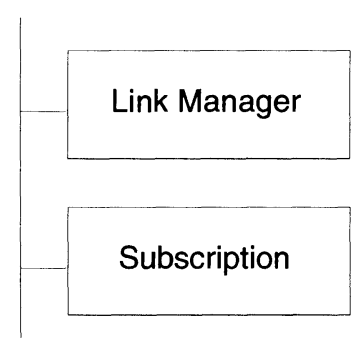

View PDB

Maintain PDB
Tool Access Cards

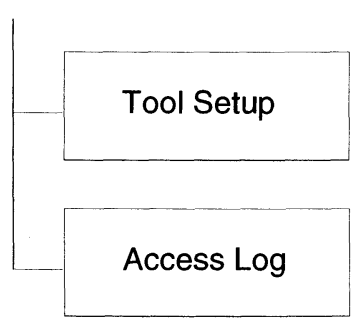

Figure 6.1 IX User Interface Structure.

The main card is basically divided into three sections: Network Access, PDB, and Tool Access. However, there are three general cards that can not be put in 
any of the above sections:

- Setup IX: This card contains general IX preferences. In this implementation it contains only the name of the IX stack.

- Messages: The message card keeps track of all messages generated by the PDB controller. A message icon appears right next to the message button whenever new messages have been generated.

- About IX: This card contains credits of this work and version numbers of the components used in the IX stack.

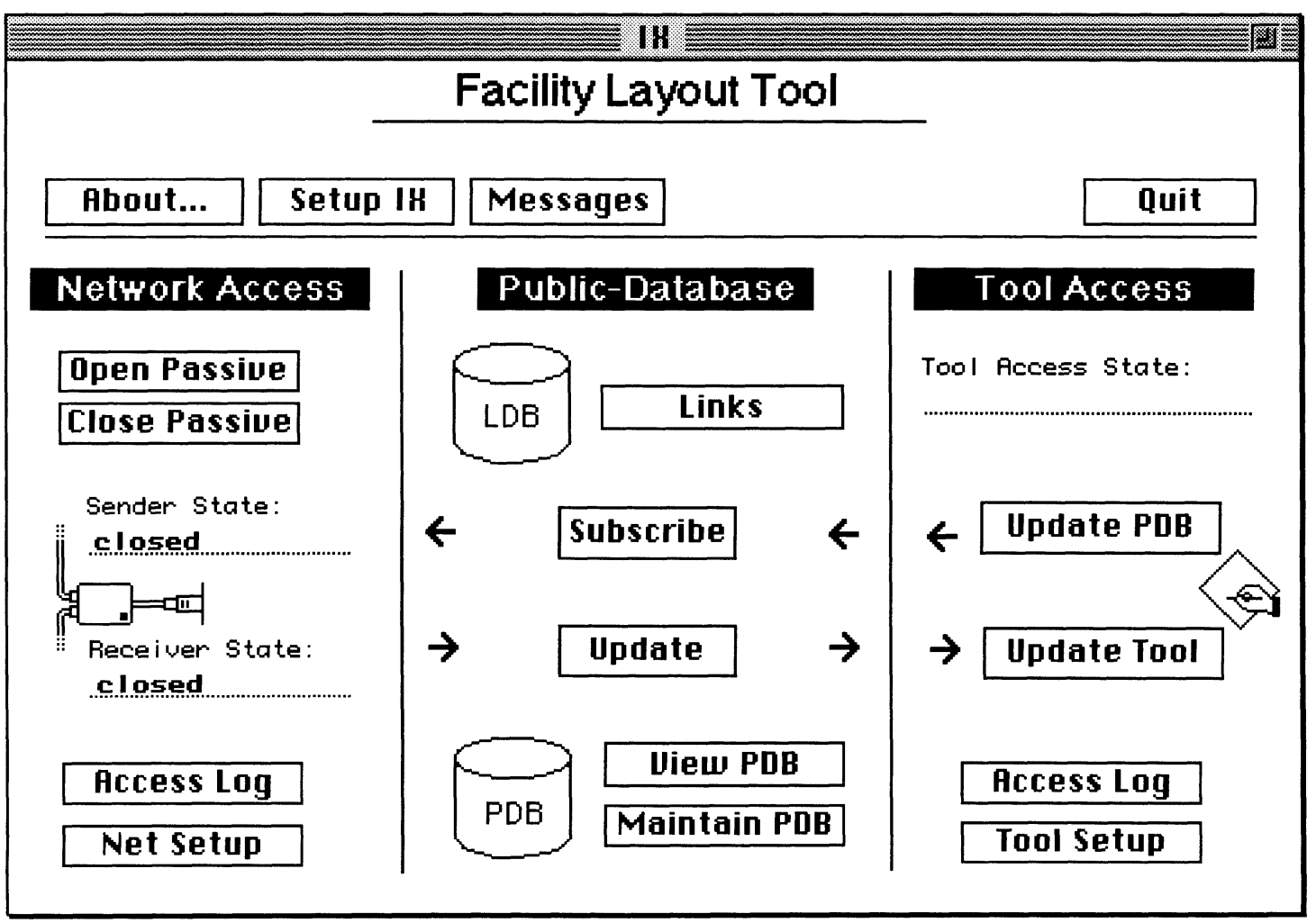

Figure 6.2 Screenshot of IX Main Card.

The following subchapters explain the user interface for each of the three main 
sections: Network Access, Public-Database, and Tool Access.

\section{Network Access}

The Network Access module needs to be set up before the IX can communicate with other IXs. The 'Net Setup' button brings up a new card that requires the user to enter the following information:

- Login-Name: This is the name used when this IX wants to connect to another IX. The login name must be one word with maximum 20 characters.

- Receiver Port: Since this implementation uses TCP/IP as communication protocol we have to specify the port number (besides the IP address) on which an IX is accessible. All IXs should use the same port number to avoid confusion.

- Node List: The user has to enter all known IP addresses of other IXs on the network. The address can be in name format (apollo.ent.edu) or number (128.0.0.13) format.

Once the network has been set up the IX is ready to communicate. There are two fields that indicate the state of the sender and the receiver. Possible values for the sender are closed and established, while the receiver has an additional listening state. The receiver is closed by default and can be opened (to listening) by pressing the 'Open Passive' button. The 'Close Passive' button brings the receiver back to the closed state. This feature allows the user to do maintenance without being disturbed by incoming requests. Finally, the access log shows 
login and logout from other IXs with date, time, and remote IX name.

\section{Public-Database}

The PDB section provides controls for the Link Manager and the PDB. The Link Manager part includes an additional card that shows all links for this particular IX and a card to subscribe data from other IXs. The link list card has controls to unsubscribe and to change the status of links manually.

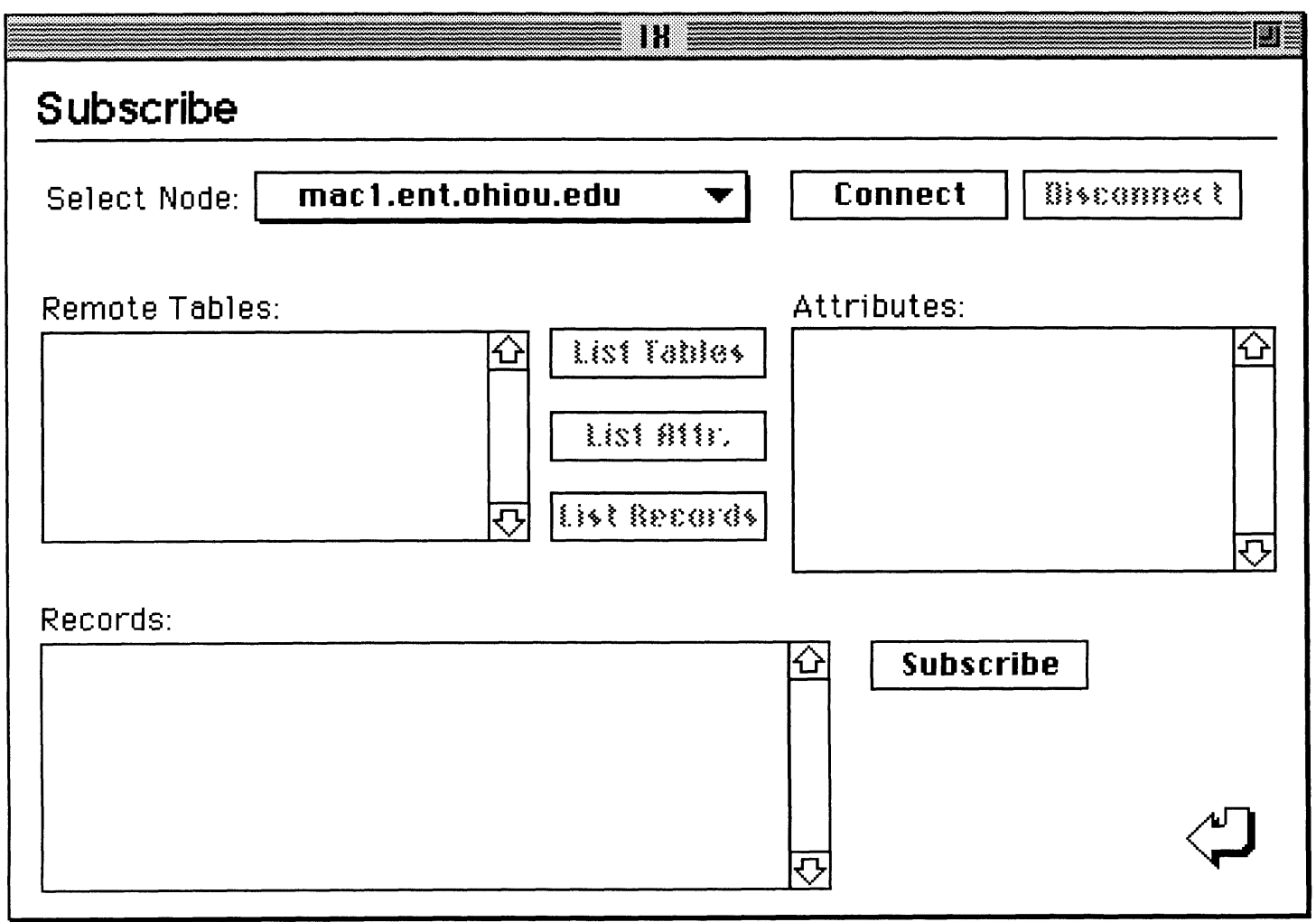

Figure 6.3 Subscription Card.

The subscription card (Figure 6.3) provides an easy way to subscribe to data 
from remote IXs. First the remote IX address has to be selected from the 'Select Node' popup menu. This menu represents the node list from the Network Setup card. A connection can be established just by pressing the 'Connect' button. A dialog box will be shown in case of a communication malfunction. Otherwise the disabled buttons (gray shaded buttons) on this card will be enabled. The user can now browse through the information stored in the remote IX's PDB. A link to the desired data will be created by pressing the 'Subscribe' button. If the connection is no longer needed, the button 'Disconnect' will properly logout from the remote IX.

Before the PDB is able store data it has to be set up according to the tool's data model. The button 'Maintain PDB' leads to a card that allows the user to specify the PDB. This includes three basic functions: create tables, remove tables, and add attributes. A future IX version may have the feature of modifying and removing attributes as well. A second button 'View PDB' lets the user browse through the PDB (see Figure 6.4). A table, selected through the popup menu, will be shown in detail (attributes type, access type, and key type). The user can also retrieve all records that are stored in the PDB for the chosen table by clicking on the 'Show Data' button. The button 'Search Data' will show only records that meet the search criteria given by the user. The search function works only within a table.

It is not possible to modify any data in the PDB manually. However, there are three automated methods to store and retrieve data to/from the PDB. One method is part of the PDB, while the two methods are part of the Tool Access. 


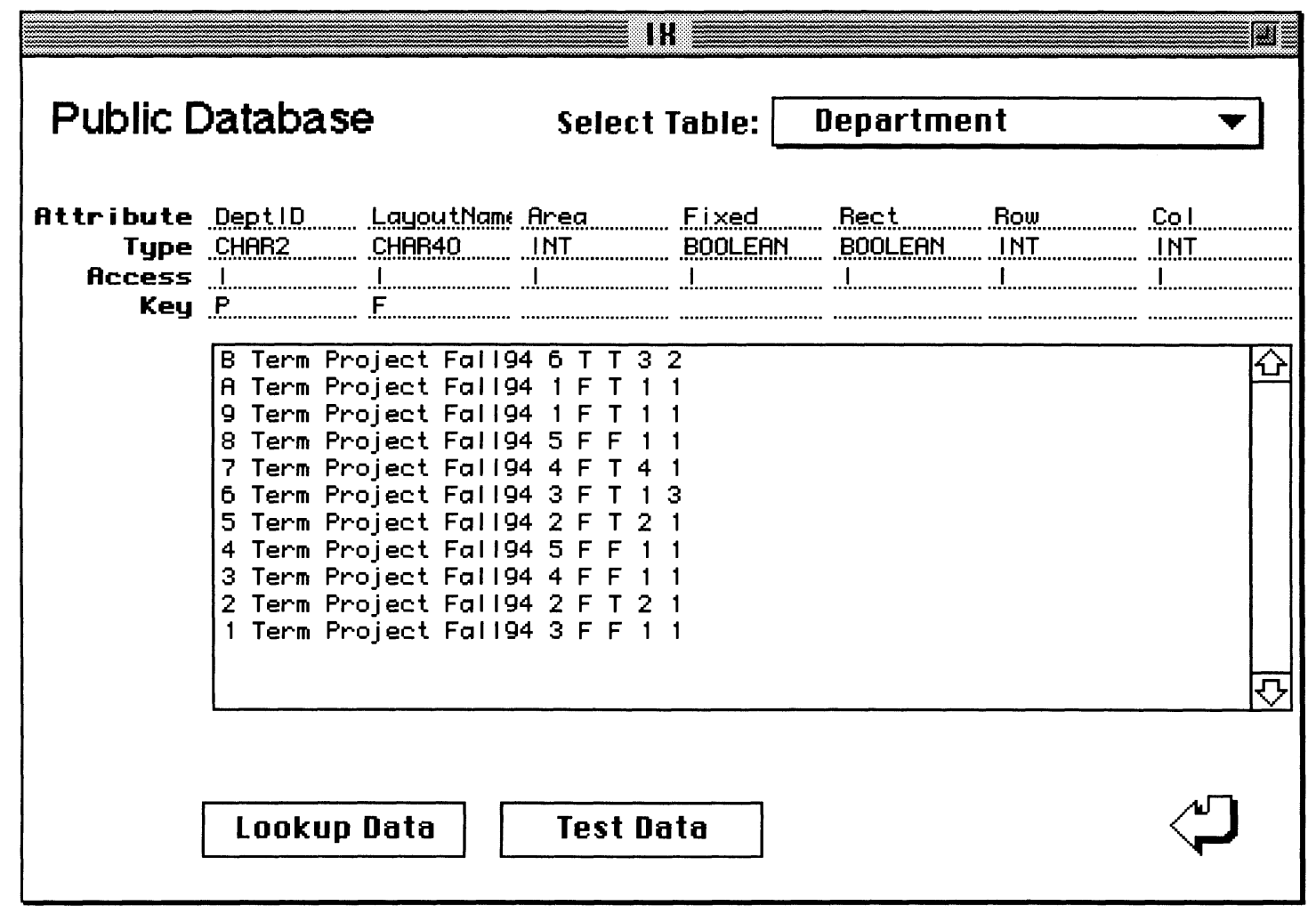

Figure 6.4 View Public-Database Card.

The 'Update' button will update the PDB based on links stored in the Link Database. Nothing will be written to the PDB if there are no links available. If data from a remote IX was been written to the PDB, the user will be notified about all modifications.

\section{Tool Access}

The Tool Access section provides configuration and update procedures for the attached tool. The contents of the 'Tool Setup' card depends on the tool and its 
communication abilities. Since all three sample applications use files for communication there is only one type 'Tool Setup' card. In this implementation translators and constructors are hardcoded in the HyperCard IX stack. Therefore, the user can only choose from different tool data files (for different projects). It is important that the selected data files correspond to the translator and constructor. For example, the implemented SIMAN translator can only process 'experiment' files. Using a SIMAN 'model' file instead, could unintentional modify the PDB.

Once the appropriate files have been selected, the tool's data can be loaded into the PDB by pressing the 'Update PDB' button. All specified tool data will be stored into the PDB. The 'Update Tool' button does the opposite by writing all data stored in the PDB back to the tool. The original tool files will be overwritten.

\section{System Requirements}

This IX implementation requires the following items:

- Any Apple Macintosh, Power Macintosh, or PowerBook personal computer

- At least 2.5 megabytes of free RAM (4 megabytes for Power Macintosh)

- Macintosh system software 7.0 or later

- MacTCP 2.0.4 or later (included in system software 7.5)

- Approximately 3.0 megabytes hard disk space for the HyperCard application and IX stack (2.5 megabytes for standalone IX application) 


\section{Sample Integration}

This chapter shows the actual integration of the three sample application by using IX's. The information that has to be exchanged between the application will be defined first. The configuration of every IX's (particularly the Tool Access) will be shown. 


\section{Sample Case Study}

In the chapter "Sample Applications" we learned about the three sample applications that are going to be used for this work. Although every application uses files to exchange data, the file formats are completely different.

The case study assumes a small plant with several departments to manufacture and to assemble products. A finished product can consist of several components (depending on the product) that are manufactured in various departments.

Figure 7.1 shows an example product component tree used in the MRP tool. The numbers in parenthesis denote the number of components needed for one final product.

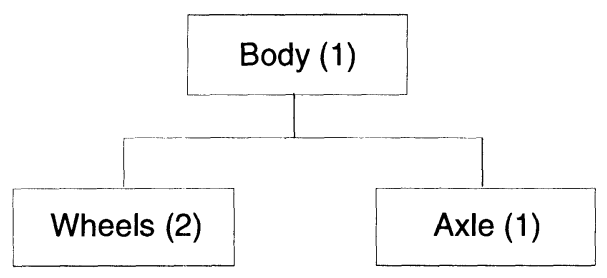

Figure 7.1 Example Product Component Tree.

The user specifies the product (components, material etc.) entirely in the MRP tool. The MRP tool keeps also other information such as workstations, orders, and employees in order to help planning and to make decisions. In this case we study only the production volume (derived from product component tree) for every component and the operation sequences (Figure 7.2) will be used from the MRP tool. The production volume has to be expressed as flow (of components) between the department necessary for production. This 
information will be used in the facility layout tool to determine an optimal layout for this particular product. The final layout contains also the eventually changed distances between departments. The distance between departments and the operation sequences from the MRP tool go into the simulation to predict product throughput time and other information of interest.

\section{Components}

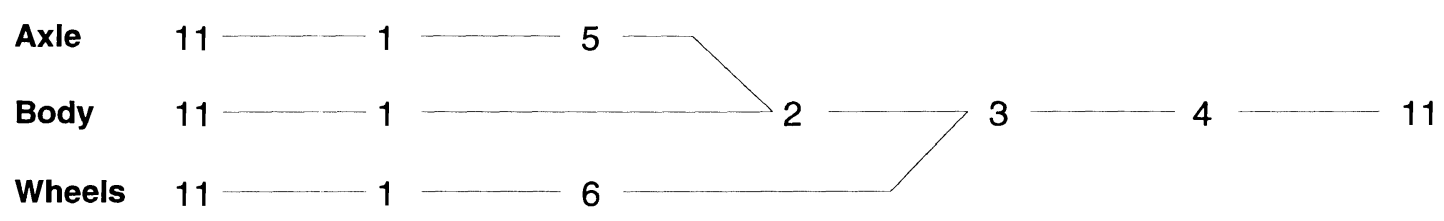

Figure 7.2 Example Product Routing through Departments.

Several cases of similar data that is used in two different tools were identified in the above text. For example, the distances between departments from the facility layout tool have to be entered in the simulation tool manually. Figure 7.3 shows the data that will be exchanged in this work.

Other data (e.g., machine operation times for each workstation from the MRP tool) could be exchanged in a more complex configuration. The simulation could also receive more data from other tools that are not used in this sample integration. 


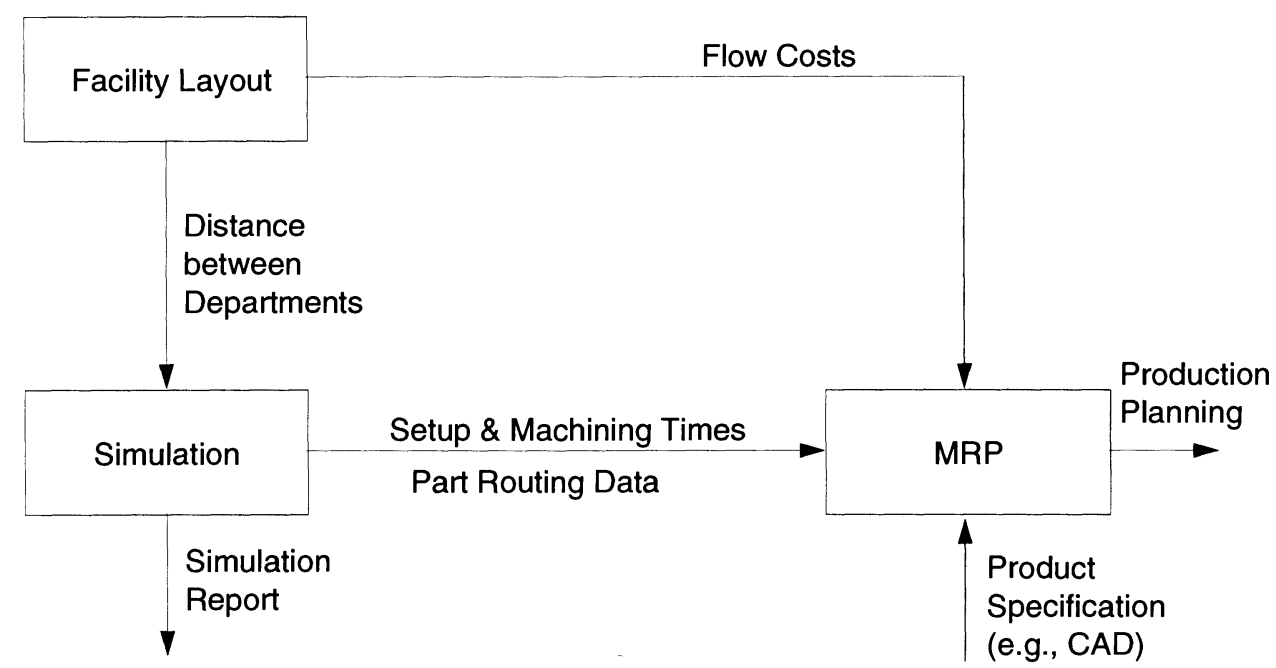

Figure 7.3 Shared Data between Sample Applications.

\section{Assumptions}

The previous section has already mentioned some assumptions about the sample integration of the three tools. The following assumptions are necessary to keep the integration uncomplicated.

- The number of departments is fixed.

- Every department consists of one workstation with a fixed operation time.

- The distance measure between departments is always rectilinear.

The variation of the number of departments and the number of workstation within a department would result in significant changes in the simulation's model. 


\section{Tool Configuration}

The next step involves the definition of the data model that will be used in the PDB. Every tool has to decide what information could be relevant for other tools and put them into the PDB. The best case would be to place all data of a tool into the PDB. However, for a complex tool (like SIMAN) it would take to much effort to come up with a complete data model. This thesis uses a complete data model only for the facility layout tool. Only the data necessary for the case study is used in the PDB for simulation tool and MRP tool.

\section{Facility Layout Tool}

The facility layout tool (QSOM) does not have any data import or data export functions built-in. However, it uses files to store all the entered layout data. The file format is not documented and had to be examined. The output file shows only the final layout drawn with ASCII characters.

Since the data model of the facility layout tool is not too complicated, it has been put entirely into the PDB (see Figure 3.4 for IDEF1X diagram). Table 7.1 lists the PDB structure.

Table 7.1 PDB Structure of Facility Layout Tool

Table

System

\section{Attribute}

LayoutName

Objective

Rows

Cols
Type

CHAR40

INT

INT

INT
Access Key

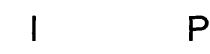




\begin{tabular}{|c|c|c|c|c|}
\hline Department & DeptID & CHAR2 & 1 & $\mathrm{P}$ \\
\hline & LayoutName & CHAR40 & 1 & $\mathrm{~F}$ \\
\hline & Area & INT & 1 & - \\
\hline & Fixed & BOOLEAN & 1 & - \\
\hline & Rect & BOOLEAN & 1 & - \\
\hline & Row & INT & 1 & - \\
\hline & Col & INT & 1 & - \\
\hline Layout & LayoutType & CHAR4 & 1 & $\mathrm{P}$ \\
\hline & LayoutName & CHAR40 & 1 & $F$ \\
\hline & Row & INT & 1 & $\mathrm{P}$ \\
\hline & Col & INT & 1 & $\mathrm{P}$ \\
\hline & Dept & CHAR2 & 1 & - \\
\hline Flow & FromDept & CHAR2 & 1 & $\mathrm{P}$ \\
\hline & ToDept & CHAR2 & I & $\mathrm{P}$ \\
\hline & LayoutName & CHAR40 & 1 & $F$ \\
\hline & Cost & REAL & 1 & - \\
\hline & Unit & INT & I & - \\
\hline & Distance & REAL & 0 & - \\
\hline
\end{tabular}

All data from the facility tool input file can be read into the PDB without any problems. However, additional translators were needed to convert the final layout and the distance between departments into the above PDB structure. The distance between departments can be obtained by calculating the centroids for every department first. By using the centroids between departments with rectilinear measurement we finally get the needed distances.

\section{Simulation Tool}

Like the facility layout tool, SIMAN does not have explicit data import or data export functions built-in. However, the input and output file structure are well documented and therefore easy to use. The model and experiment files were taken from a previous class project (Dreer 1994) that examined the relationship 
between flow cost and throughput time.

According to the sample integration description only elements of the experiment file (.exp) are stored into the PDB. Table 7.2 lists the PDB structure.

Table 7.2 PDB Structure of Simulation Tool

Table

System

Sequence

Distance
Attribute

ProjectName

Author

PartNo

ProjectName

Order

Station

SetNo

ProjectName

FromStation

ToStation

Distance
Type

CHAR40

CHAR40

INT

CHAR40

INT

CHAR4

INT

CHAR40

CHAR4

CHAR4

INT
Access Key

P

P

F

P

$\mathrm{P}$

F

$\mathrm{P}$

$\mathrm{P}$

\section{MRP Tool}

The MRP offers a data export utility that is used to produce several files with the information needed for other tools. The following ASCII files were exported (with fixed filename given by the tool): BOM.ASC, MAC.ASC, OPE.ASC, LEAD.ASC, and LDTIME.ASC. The file format of every export file is well described in (Chong 1989). 
Table 7.3 PDB Structure of MRP Tool

\begin{tabular}{|c|c|c|c|c|}
\hline Table & Attribute & Type & Access & $\mathrm{Ke}$ \\
\hline \multirow[t]{2}{*}{ Part } & PartID & CHAR8 & 1 & $P$ \\
\hline & Description & CHAR32 & $i$ & - \\
\hline \multirow[t]{3}{*}{ Component } & PartID & CHAR8 & 1 & $F$ \\
\hline & ComplD & CHAR8 & i & $\mathrm{F}$ \\
\hline & UsageRate & INT & I & - \\
\hline \multirow[t]{4}{*}{ Route } & PartID & CHAR8 & 1 & $F$ \\
\hline & OpID & CHAR6 & I & $\mathrm{F}$ \\
\hline & Order & INT & 1 & $\mathrm{P}$ \\
\hline & OpTime & REAL & 1 & - \\
\hline \multirow[t]{3}{*}{ Operation } & OpID & CHAR6 & 1 & $\mathrm{P}$ \\
\hline & Description & CHAR32 & 1 & - \\
\hline & MachinelD & CHAR6 & 1 & - \\
\hline \multirow[t]{2}{*}{ Machine } & MachinelD & CHAR6 & 1 & $P$ \\
\hline & Description & CHAR32 & 1 & - \\
\hline
\end{tabular}

The IX for the MRP tool requires only a translator, since no data from other tools is read into the MRP tool (at least in this implementation). 


\section{Conclusions}

This chapter concludes this thesis. It discusses the accomplishments made as well as limitations and problems that have occurred during design and development. Finally, further extensions and improvements of this work will be shown. 


\section{Accomplishment}

This thesis provides a feasible solution to integrate data between tools and applications that may not be necessarily designed to share their data. The IX concept does not require a central data server system. Every tool maintains its independency and can share and receive data from other components. The internal Public-database forces every tool to store a copy of its data in a neutral format, which is not tool dependent. The data conversion into the PDB requires some extra effort, however, the advantage is that other tools can easier access the tool's data.

Another important integration part is represented by the communication model. The IX-P offers system independent communication preferably used in TCP/IP environments. IX-P is a simple and transparent protocol that can be both easily ported to other platforms and extended with other necessary commands (e.g., password to login into an IX).

The usage of static translations (filters) have already been discussed in the introduction. The IX approach has some advantages over a filter:

- communication over network

- multi-user access

- querying of published application data

Filters are usually used to translate data between two tools from one data format to another. This translation is normally hardcoded and works only for the two 
particular tools. A new filter has to be written if the data is also needed in another (third) tool. In the IX approach the data of the attached tool is translated once (into the PDB) and can then be accessed by other applications (through the IX interface). Since this implementation does not support any version control system it is necessary to translate all tool data whenever another IX requests any data. Multiple users (IX nodes) can access one particular IX depending on the number of available listener sockets.

The IX concept works like a network of distributed databases. This makes it possible to send queries to any IX and view or subscribe to data from any tool, which is not possible by using filters. Basically CIM tools with built-in database access (e.g., an integrated SQL database engine) and network communication features would provide a similar integration to the IX approach.

\section{Limitations and Problems}

The present implementation demonstrates an basic integration of three tools, however, there are a few limitations that could be eliminated or changed in future implementations. Most problems and limitations can be solved simply by investing more time and may be considered as further extension (see next section 'Further Research').

Right now the IX is based on the HyperCard environment, which is only available for Apple Macintosh computers. HyperCard applications have quite some overhead and may not be the final development environment for an 
'industrial' implementation. However, it allows easy and fast development, which is important during prototyping.

The PDB offers basic commands and functions to manage data. Additional functions such as modification of an attribute or remove attribute could be added. Another important PDB issue is its performance. This is especially true for most search and data retrieval functions.

This implementation uses 'hard-coded' translators and constructors for every tool by using the HyperTalk language. This is a feasible way to do it, however, the usage of a sophisticated translation mechanism with translation tables would simplify the configuration procedure.

The current implementation of the Link Manager links one instance of an IX to an instance of another IX. A link consists of all key attributes necessary to identify the instances. However, this method presents inconsistency problems when adding new records or deleting existing records and is very inefficient for large tables.

Another solution would be to define entity-to-entity relationships. This approach requires only one link to bridge two tables from two different IXs together. A synchronization procedure ensures consistency between both entities. However, this method imposes certain restrictions that have to be considered during modeling of the PDB. For example, the key attributes should be the same for both entities. Some problems come up when the key attributes are the same for both entities, but their semantic meaning is different (e.g., one 
entity uses department numbers, the other entity uses department names). In this case, additional translation tables must be provided int the local IX (see also next section).

\section{Further Research}

This thesis offers many opportunities for further research. Some suggestions have already been mentioned throughout this document. The following list shows some possible IX additions:

- IX implementations on other platforms such as Intel-based PCs or UNIXbased workstations

- Tool Access modules (including translator/constructor) for other CIM applications

- Tool Access module for SQL databases

- Tool independent translation and construction mechanism by using internal translation tables

The next recommendations are not necessarily more difficult, however, they involve more conceptual work than the above suggestions.

All tools that are integrated through IXs basically publish their data in a neutral format through the local PDB. However, the contents of a PDB depends on the tool's data model that is actually used in the particular PDB. The IX-P allows any node on the network (preferably TCP/IP) to request an IX's detailed data 
model that is stored in the PDB. Therefore, it is possible to generate a reflection of all IX's together, which could be interpreted as an up-to-date enterprise-wide data model.

Another major issue is the semantic interoperabiliy for all integrated tools. For example, the facility layout tool uses the term 'flow cost' for the units transported from one to another department, while the MRP tools calls the same attribute 'production volume'. Similar problems occur when data needs to be scaled or converted into another unit. An interesting article (Sciore et al. 1994) discusses these kind of problems and shows also possible solutions to ensure semantic interoperability. The IX concepts may be expanded with new information system components that take care of this problem.

The problems of the current implemented Link Manager have already been discussed in the previous section. Every entry in the link database links exactly one attribute value to another IX by specifying its table and key. A link of another value requires a new link which can result in several entries in the link database. For example, all distances between departments from the facility layout tool are linked to the simulation tool with one link entry for every distance. Thus, a facility layout with 10 departments requires 90 links. A more intelligent link manager would have only one entry that includes the information necessary to link all 90 entries. 


$\begin{array}{ll}\text { ALAP } & \text { AppleTalk Link Access Protocol } \\ \text { ASCII } & \text { American Standard Code for Information Interchange } \\ \text { ANSI } & \text { American National Standards Institute } \\ \text { BOM } & \text { Bill of Materials } \\ \text { CAD } & \text { Computer Aided Design } \\ \text { CAE } & \text { Computer Aided Engineering } \\ \text { CAM } & \text { Computer Aided Manufacturing } \\ \text { CAP } & \text { Computer Aided Planning } \\ \text { CAPP } & \text { Computer Aided Process Planning } \\ \text { CAQ } & \text { Computer Aided Quality Assurance } \\ \text { CIM } & \text { Computer Integrated Manufacturing } \\ \text { CIM-OSA } & \text { CIM Open System Architecture } \\ \text { CNC } & \text { Computerized Numerical Control } \\ \text { CRAFT } & \text { Computerized Relative Allocation of Facilities Technique } \\ \text { CSMA } & \text { Carrier-Sense Multiple-Access } \\ \text { DFD } & \text { Data Flow Diagram } \\ \text { DXF } & \text { Data Interchange Format } \\ \text { EDI } & \text { Electronic Data Interchange } \\ \text { ERD } & \text { Entity Relationship Diagram } \\ \text { FIPS } & \text { Federal Information Processing Standard } \\ \text { FMS } & \text { Flexible Manufacturing System } \\ \text { FTAM } & \text { File Transfer, Access and Management } \\ \text { FTP } & \text { File Transfer Protocol } \\ \text { GT } & \text { Group Technology } \\ \text { ICAM } & \text { Integrated Computer Aided Manufacturing } \\ \text { IDEF } & \text { ICAM Definition Method } \\ \text { IDEM } & \text { Integrated Design and Modeling } \\ \text { IEEE } & \text { Institute of Electrical Electronics Engineers } \\ \text { /O } & \text { Input/Output } \\ \text { ISO } & \text { International Organization for Standardization } \\ \text { IX } & \text { Integration Extension } \\ \text { IX-P } & \text { IX Protocol } \\ \text { LAN } & \text { Local Area Network } \\ \text { MAP } & \text { Manufacturing Automation Protocol } \\ \text { MHS } & \text { Message Handling System } \\ \text { MMS } & \text { Manufacturing Message Specification } \\ \text { MRP } & \text { Material Requirement Planning } \\ \text { MRP2 } & \text { Manufacturing Resources Planning } \\ \text { NBP } & \text { Name Binding Protocol } \\ \text { NC } & \text { Numerical Control } \\ \text { NDM } & \text { Normal Disconnected Mode } \\ \text { NRM } & \text { Normal Response Mode } \\ \text { NIST } & \text { National Institute of Standards and Technology } \\ \text { OSI } & \text { Open Systems Interconnection } \\ \text { PDB } & \text { Public Database } \\ \text { PC } & \text { Personal Computer } \\ \text { PLC } & \text { Programmable Logical Controllers } \\ \text { PPC } & \text { Production Planning and Control } \\ & \end{array}$




$\begin{array}{ll}\text { QSOM } & \text { Quantitative Systems for Operations Management } \\ \text { SADT } & \text { System Analysis and Design Technique } \\ \text { SED } & \text { State-Event Diagram } \\ \text { SMTP } & \text { Simple Mail Transfer Protocol } \\ \text { SNA } & \text { System Network Architecture } \\ \text { SPARC } & \text { Standards Planning and Requirements Committee } \\ \text { SQL } & \text { Structured Query Language } \\ \text { TOP } & \text { Technical Office Protocol } \\ \text { XCMD } & \text { HyperCard External Command } \\ \text { XFCN } & \text { HyperCard External Function }\end{array}$




\section{References}

This section contains all references that have been mentioned in this proposal.

Books and articles were mostly from the Alden library at Ohio University, Athens and the Federal Institute of Technology (ETHZ) library in Zürich, Switzerland. 
Apple Computer, Inc. HyperCard 2.3 - Reference Manual. Cupertino: Apple Computer, Inc., 1993.

Apple Computer, Inc. HyperCard 2.3 - HyperTalk Script Manual. Cupertino: Apple Computer, Inc., 1994.

Bedworth, David D., Mark R. Henderson, and Philipp M. Wolfe. Computer Integrated Design and Manufacturing. New York: McGraw-Hill, 1991.

Bourdeau, Richard. "The Role of Distributed Databases in Integrated Manufacturing Solutions.” Digital Equipment Corp., CIM Marketing and Product Development, 1989.

Chong, Philip S. MRP DSS - Manufacturing Resource Planning Decision Support System. Computer Software. Version 4.01. Advance MicroPower International, Fountain Valley, CA, 1989.

Compton, W. Dale., ed. Design and Analysis of Integrated Manufacturing Systems. Washington, DC: National Academy Press, 1987.

Cypser, R.J. Communications for Cooperating Systems OSI, SNA. and TCP/IP. Addison-Wesley Publishing, Inc., 1991.

Dato, M. Actis. "Standards for Manufacturing." Computer Integrated Manufacturing - Perspectives for International Economic Development and Competitiveness. United Nations Economic Commission for Europe, Geneva, 1991.

Dickson, Gary and Alan Lloyd. Open Systems Interconnection. Australia: Prentice Hall Pty Ltd, 1992.

Dreer, Pascal. "Relationship Between Flow Cost and Throughput Time." Ohio University, ISE640 Class Project, 1994.

Eirich, Peter., ed. "Enterprise Modeling: Issues, Problems \& Approaches" International Conference on Enterprise Modeling Technology (ICEIMT), South Carolina, June 6-10, 1992.

Fox Mark S., John F. Chionglo, Fadi G. Fadel. “A Common-Sense Model of the Enterprise" Proceedings of the 2nd Industrial Engineering Research Conference, 1993: 425-429.

FIPS183. Integrated Definition for Function Modeling (IDEF0). Federal Information Processing Standards Publication 183, Department of Commerce, 1993.

FIPS 184. Integrated Definition for Information Modeling (IDEF1X). Federal Information Processing Standards Publication 184, Department of Commerce, 1993. 
Ganguin, D. Das CIM Unternehmen. München: IBM Deutschland GmbH, Industrie und Technik, 1990.

Jorysz, H.R. and F.B. Vernadat. "CIM-OSA Part 1: Total Enterprise Modelling and Function View." International Journal of Computer Integrated Manufacturing 3.3 (1990): 144-156.

Jorysz, H.R. and F.B. Vernadat. "CIM-OSA Part 2: Information View." International Journal of Computer Integrated Manufacturing 3.3 (1990): 157-167.

Klittich, M. "CIM-OSA Part 3: CIM-OSA Integrating Infrastructure the Operational Basis for Integrated Manufacturing Systems." International Journal of Computer Integrated Manufacturing 3.4 (1990): 168-180.

Malhorta, Rajeev and Sundaresan Jayaraman. "An Integrated Framework for Enterprise Modeling." Journal of Manufacturing Systems 11.6 (1993): 426-441.

Mackiewic, Ralph. An Overview to the Manufacturing Message Specification. World-Wide Web: http://litwww.epfl.ch/ mms/MMSIntroSISCO, 1994.

Nicholson, Harold. Structure of Interconnected Manufacturing Systems. London: Peter Peregrinus Ltd., 1991.

Nof, Shimon Y., ed. Information and Collaboration Models of Integration. NATO ASI Series, Kluwer Academic Publishers, 1993.

O’Grady, P.J. Controlling Automated Manufacturing Systems. New York, NY: Chapman and Hall, 1986.

Öszu, Tamer M. and Patrick Valduriez. Principles of Distributed Database Systems. Englewood Cliffs, NJ: Prentice-Hall, Inc., 1991.

Pato, T., and H. Schwarzenbach. "CIM Einführung." Lecture at HTL Bern, Sommer 1990.

Pedgen, C. Dennis, Robert E. Shannon, and Randall P. Sadowski. Introduction to Simulation Using SIMAN. McGraw-Hill, Inc., 1990.

Pimentel, Juan R. Communication Networks for Manufacturing. Englewood Cliffs, NJ: Prentice-Hall, Inc., 1990.

Rangan, Ravi M. and Robert E. Fulton. "A Data Management Strategy to Control Design and Manufacturing Information." Engineering with Computers 7 (1991): 63-78. 
Reddy, M.P., et al. "A Methodology for Integration of Heterogeneous Databases." IEEE Transactions on Knowledge and Data Engineering 6.6 (1994): 920-933.

Rembold, U., B.O. Nnaji, and A.Storr. Computer Integrated Manufacturing and Engineering. Addison-Wesley Publishers Ltd., 1993.

Scheer, A.-W. Enterprise-Wide Data-Modelling. Berlin: SpringerVerlag, 1989.

Scheer, A.-W. CIM Computer Steered Industry. Berlin: SpringerVerlag, 1988.

Sciore, Edward., Michael Siegel, and Arnon Rosenthal. "Using Semantic Values to Facilitate Interoperability Among Heterogeneous Information Systems." ACM Transactions on Database Systems 19.2 (1994):254-290.

Sheth, A. and J. Larson. "Federated Database Systems for Managing Distributed, Heterogeneous, and Autonomous Databases." ACM Computing Surveys 22.3 (1990): 183-236.

Sriram D., S. Ahmed, and R. Logcher. "A Transaction Management Framework for Collaborative Engineering." Engineering with Computers 8 (1992): 213-232.

Teorey, Toby J. Database Modeling \& Design: The Fundamental Principles. 2nd ed. San Francisco, CA: Morgan Kaufmann Publishers, Inc., 1994.

Wang, W., K. Popplewell and R. Bell. "An Integrated Multi-view System Description Approach to Approximate Factory Modelling." International Journal of Computer Integrated Manufacturing 6.3 (1993): 165-174.

Weatherall, Alan. Computer Integrated Manufacturing - A total competitive strategy. 2nd ed. Oxford: Butterworth-Heinemann Ltd, 1992.

Zehnder, C.A. Informationssysteme und Datenbanken. 5th ed. Stuttgart: B.G. Teubner, 1989.

Zhang, Hong-Chao and Leo Alting. Computerized Manufacturing Process Planning Systems. London: Chapman \& Hall, 1994. 


\section{Appendix}
A IX HyperTalk Program Listings
B IX External Command Program Listings 


\section{A IX HyperTalk Program Listings}

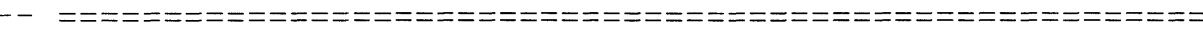

-- Main HyperTalk programs for IX application.

$--$

-- Author:

-- Pascal Dreer

$--$

-- Part of MS Thesis (August 1995)

$--$

$--$

-- Language:

$--$ HyperTalk (Macintosh)

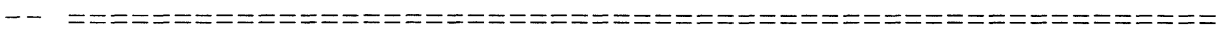

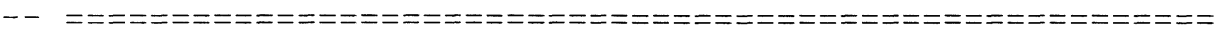

- Program Startup and Quit Functions

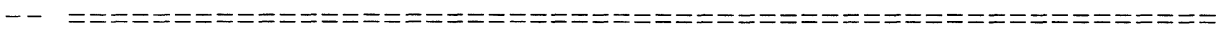

on openstack

global gHCPath

NetworkInit

ToolInit

end openstack

on idle

NetworkPassiveRecv

end idle

on closestack

NetworkDispose

end closestack

function OpenHCFolderFile shortName

get the long name of HyperCard

put it \& ":" \& shortName into it

open file it

--put it into the result

--get the result

return it

end OpenHCFolderFile

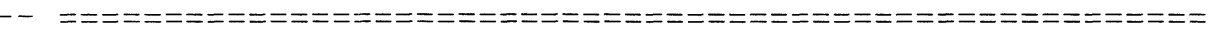

- NETWORK ACCESS MODULE (protocol dependent)

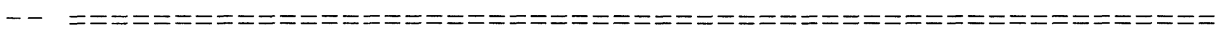

on NetworkInit 
global gConnInID, gConnOutID, gPort, gCommMode, gNodeList, gOwnAddr

put empty into gConnInID

put empty into gConnOutID

put "closed" into gCommode

put field "port" of card "netsetup" into gPort

put field "nodes" of card "netSetup" into gNodeList

put field "ownAddr" of card "netSetup" into gOwnAddr

put "closed" into field "sender" of card "main"

put "closed" into field "receiver" of card "main"

end NetworkInit

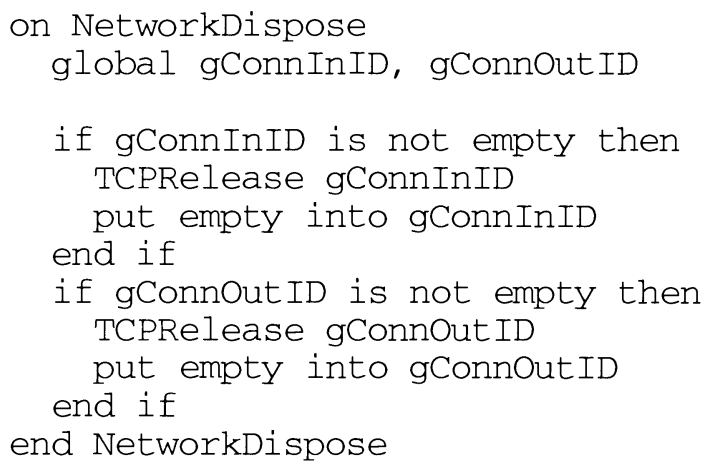

on NetworkActiveopen address

global gConnOutID, gPort

put TCPNameToAddr(address) into it

put TCPActiveopen(it,gPort, 0) into gConnOutID

if gConnOutID contains "fail" then answer "Couldn't open active connection." with OK put empty into gConnOutID put "closed" into field "sender" of card "main" end if

end NetworkActiveOpen

on NetworkPassiveOpen

global gConnInID, gPort, gCommMode

if gConnInID is empty then

put TCPPassiveopen $(0,0$, gPort $)$ into gConnInID

if gConnInID contains "fail" then

answer "Couldn't open passive connection." with OK

put empty into gConnInID

put "closed" into gCommode

else

put "listening" into field "receiver" of card "main" put "NC" into gCommMode

end if 
else

answer "Passive connection is already open" with OK end if

end NetworkPassiveopen

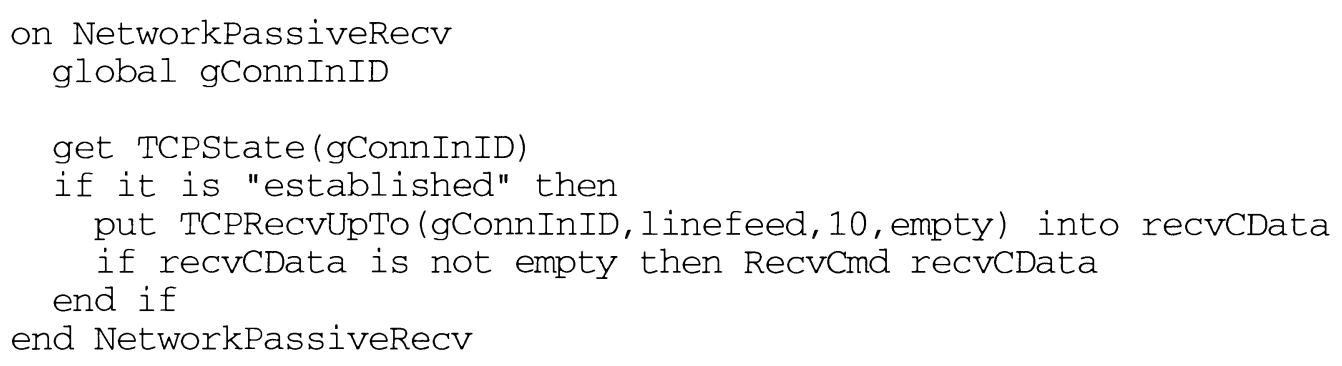

on NetworkSend connID, sendData

get TCPState (connID)

if it is "established" then

TCPSend connID, sendData end if

end Networksend

on NetworkPassiveclose

global gCommMode, gConnInID

if gConnInID is not empty then

TCPClose gConnInID

TCPRelease gConnInID

put empty into gConnInID

put "closed" into gCommMode 


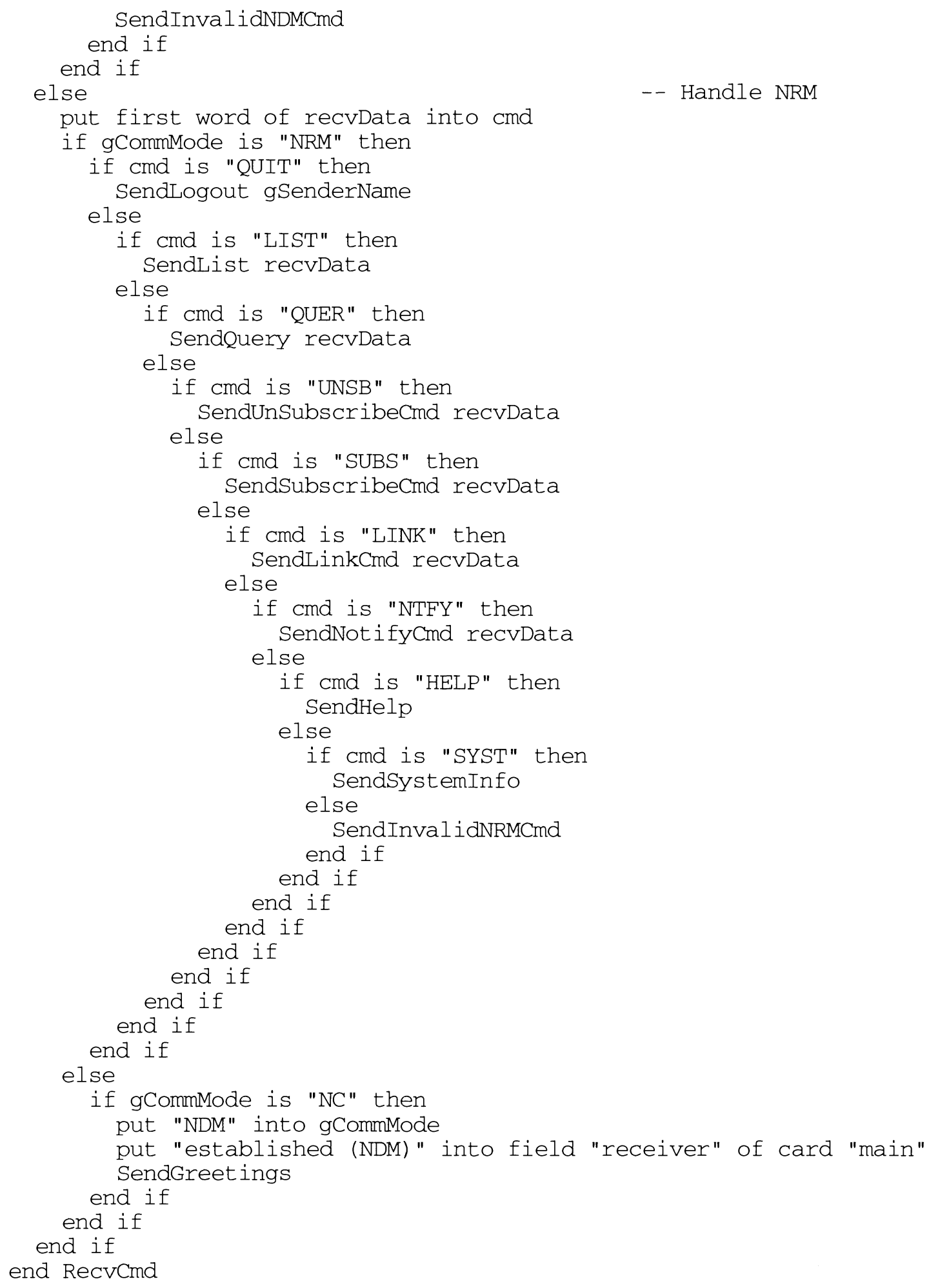

on RecvReply1 recvData 
put first word of recvData into replycode

if replycode is "100" then

put bg field "loginName" of card "netsetup" into it

SendCmaUser it

end if

if replycode is "110" then

answer "Login accepted" with "OK"

end if

if replycode is "111" then

answer "Login access denied" with "OK"

end if

if replycode is "112" then

answer "Invalid Command" with "OK"

end if

end RecvReply1

on RecvReply2 recvData

put first word of recvData into replycode

if replycode is "200" then

put recvData into field "remoteTables" of card "subscribe" end if

end RecvReply2

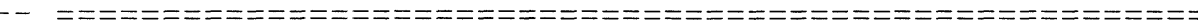

-- NETWORK SENDER MODULE

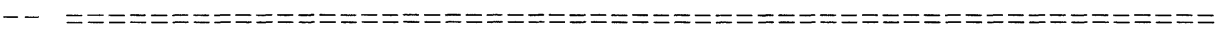

on SendReply sendData

global gConnInID

put return \& linefeed \& numToChar(3) after sendData -- ETX Networksend gConnInID, sendData

end SendReply

on sendCmd sendData

global gConnOutID

put return \& linefeed after sendData

Networksend gConnOutID, sendData

end SendCmd

-- Send Replies

- - - -

on SendGreetings

put "100 IX Version $1.0 d$ ready. Send user ID." into it

SendReply it

end SendGreetings 


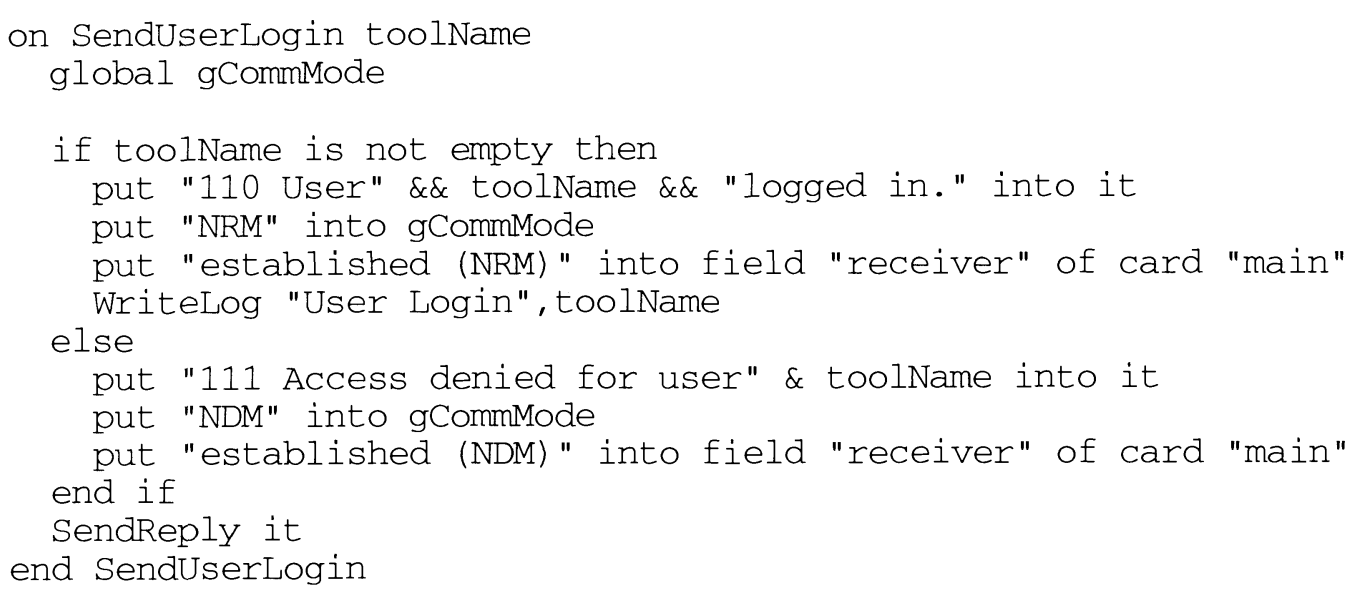

on SendHelp

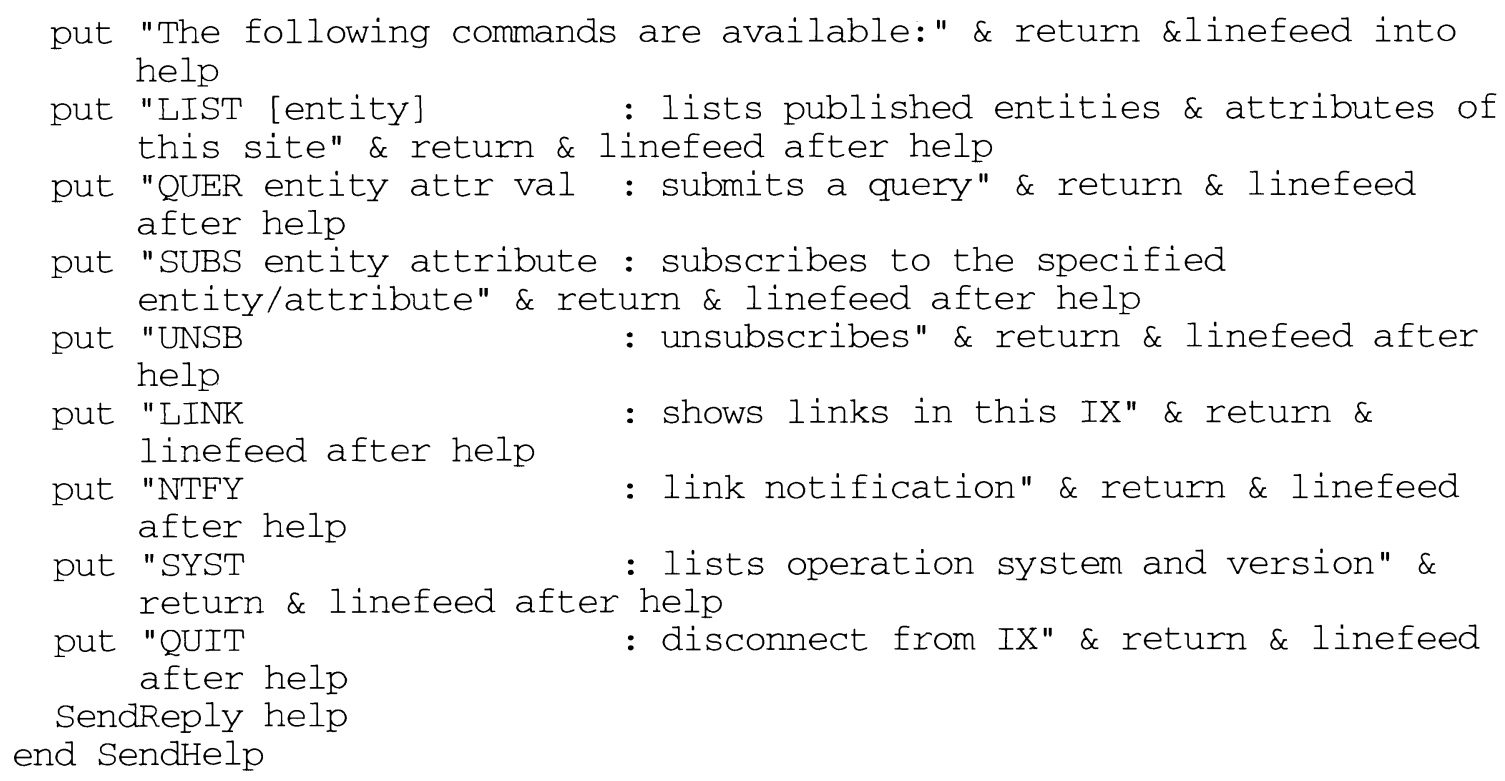




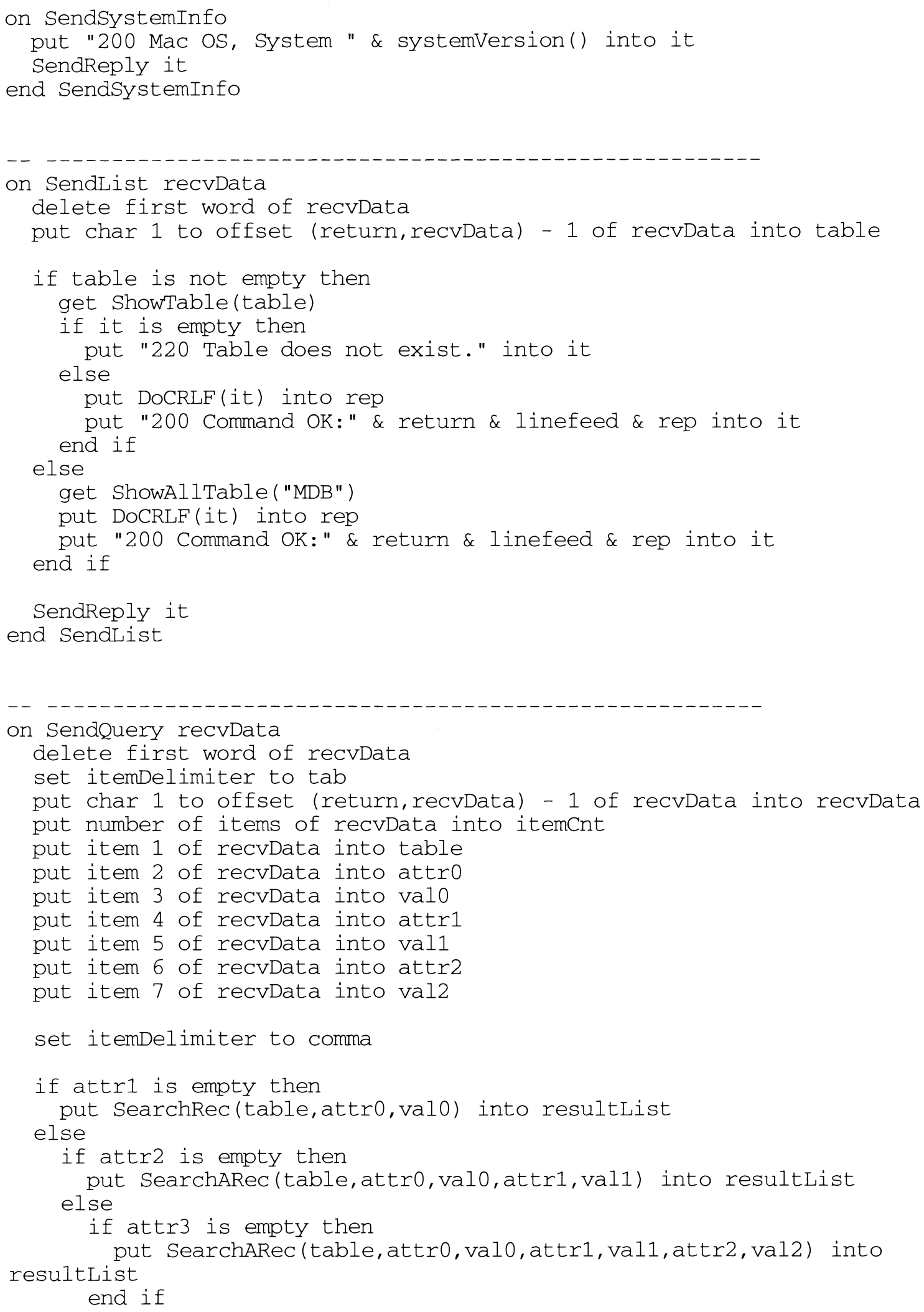




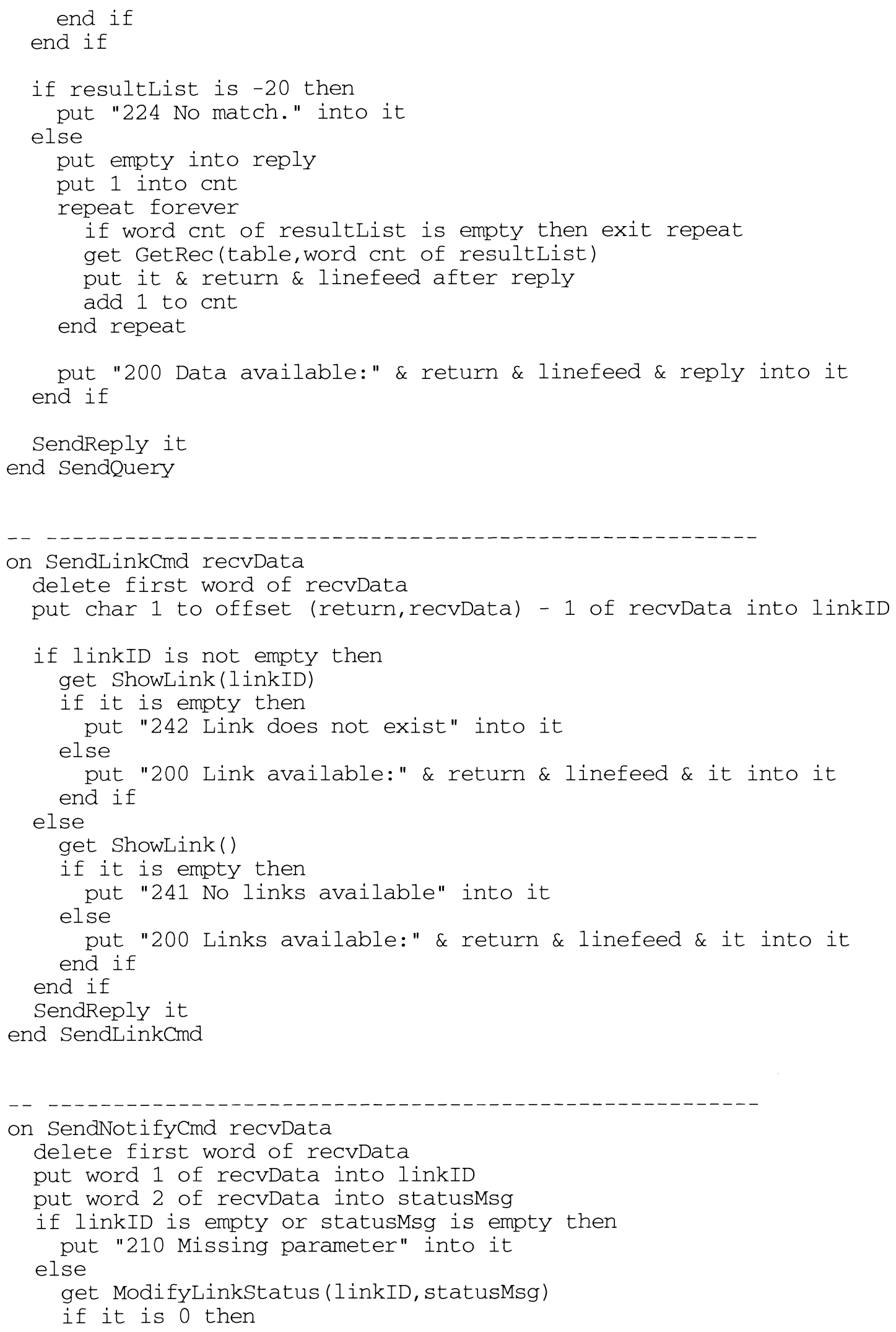


put "200 Link Status changed" into it

else

put "242 Link does not exist" into it end if

end if

SendReply it

end SendNotifyCmd

on SendSubscribecmd recvDat:a

delete first word of recrData

put word 1 of recvData into nodeAddr

put word 2 of recvData into rLinkID

put word 3 of recvData into table

put word 4 of recvData into attr

put word 5 of recvData into key

put GetNewLinkID() into localLinkID

get AddLink (nodeAddr, rLirikID, localLinkID, "P" , table, attr, key)

if it is 0 then

put "200 Subscription OK" \& return \& linefeed \& localLinkID into it else

put "240 Subscription failed" into it

end if

SendReply it

end SendSubscribecmd

on SendUnSubscribecmd recvilata

delete first word of recviata

put word 1 of recvData into linkID

get RemoveLink(linkID)

if it is 0 then

put "200 Unsubscription successful" into it else

if it is 1 then

put "242 Link does not exist" into it

else

put "240 Unsubscription failed" into it end if

end if

SendReply it

end SendUnSubscribecmd

on SendInvalidNDMCmd

put "112 Invalid Command in NDM mode (Use USER or QUIT) " into it

SendReply it

end SendInvalidNDMCmd 
on SendInvalidNRMCmd

put "211 Invalid Command in NRM mode (Use HELP for available commands) " into it

SendReply it

end SendInvalidNRMCmd

on SendNot Implemented

put "212 Command not implemented" into it

SendReply it

end SendNot Implemented

- - Send Commands

- - - - - - - - - - -

function ConnectIX address

global gConnOutID

NetworkActiveopen address

if NetworkState(gConnOutID) is "established" then

put "established" into field "sender" of card "main"

put return \& linefeed into it

Networksend gConnOutID, it

get NetworkActiveRecv()

SendCmaUser field "loginName" of card "netSetup"

get NetworkActiveRecv()

if word 1 of first line of it is 111 then

answer "User login invalid." with "OK"

DisconnectIX

return false

end if

if word 1 of first line of it is 110 then

return true

end if

else

NetworkActiveclose

answer "Connection failed." with "OK"

return false

end if

end ConnectIX

on DisconnectIX

global gConnOutID

if NetworkState(gConnOutID) is "established" then

SendCmdQuit

get NetworkActiveRecv()

NetworkActiveclose

end if

end DisconnectIX 


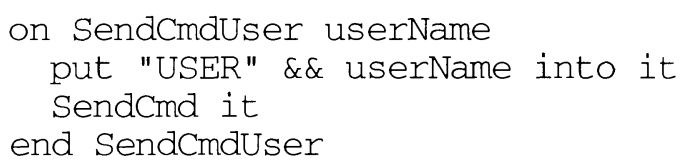

on SendCmdNotify linkID, statusMsg

put "NTFY" \&\& linkID \&\& statusMsg into it SendCmd it

end SendCmaNotify

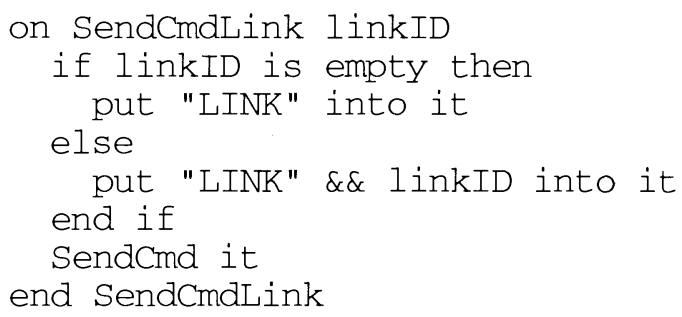

on SendCmaSubscribe linkID, table, attr, key global gOwnAddr

put "SUBS" \&\& gOwnAddr \&\& linkID \&\& table \&\& attr \&\& key into it sendCmd it end SendCmdSubscribe

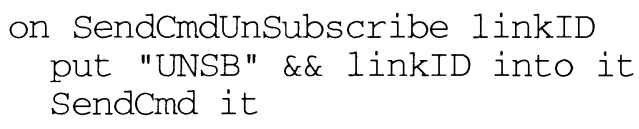


end SendCmaUnSubscribe

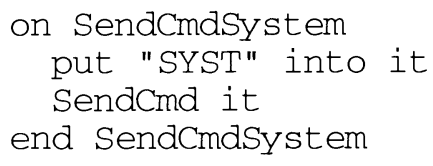

on WriteLog logType, logText

get OpenHCFolderFile("IX AccessLog")

put it into refNum

put the short date \&\& the long time \& tab \& logType \& " : " \& logText \& return into it

write it to file refNum at end

close file refNum

end WriteLog

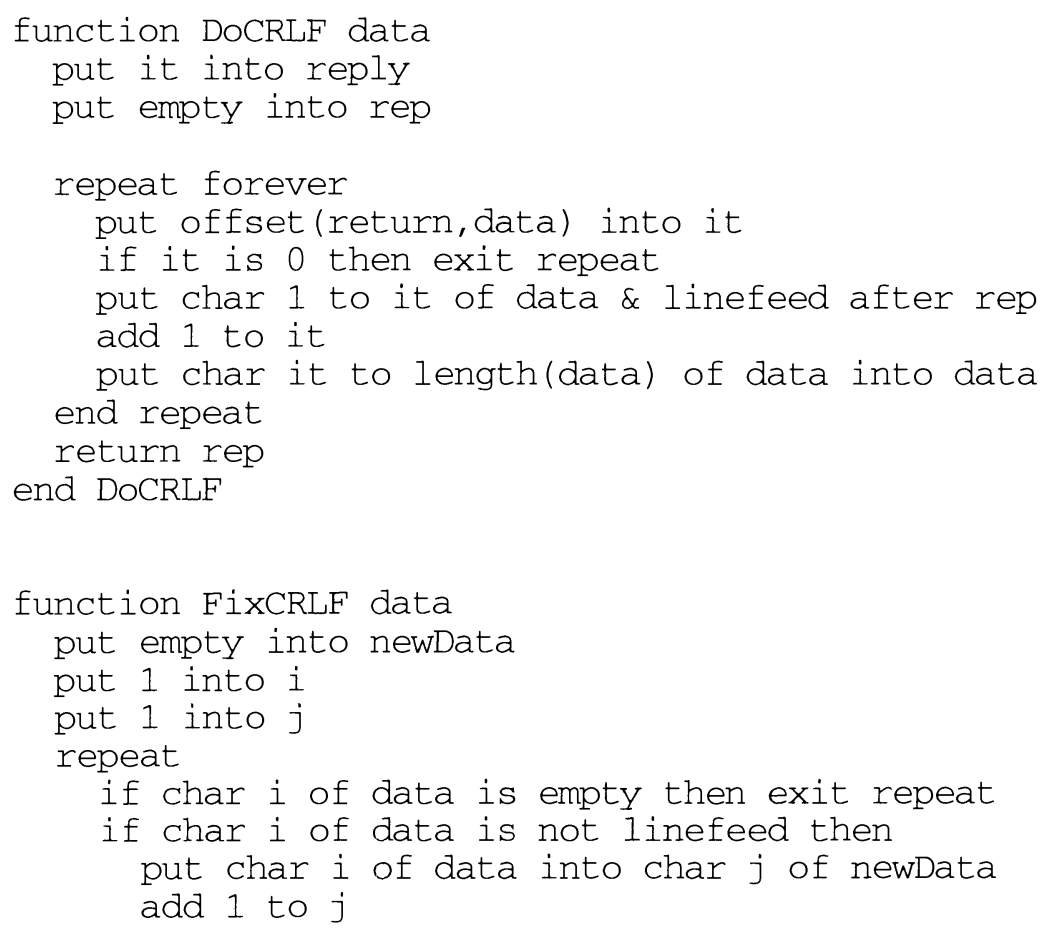

function FixCRLF data put empty into newData 
end if

add 1 to $i$

end repeat

return newData

end FixCRLF

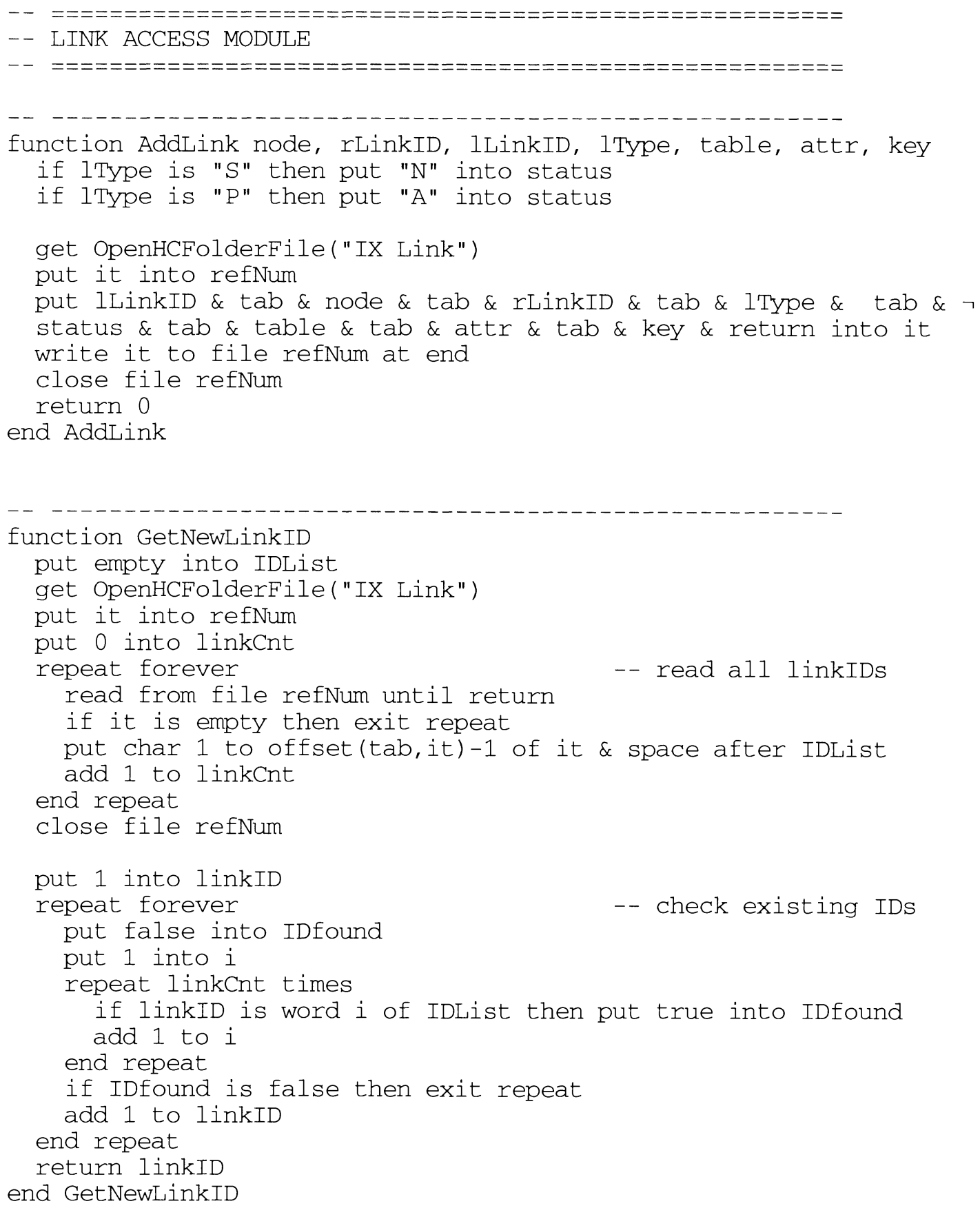




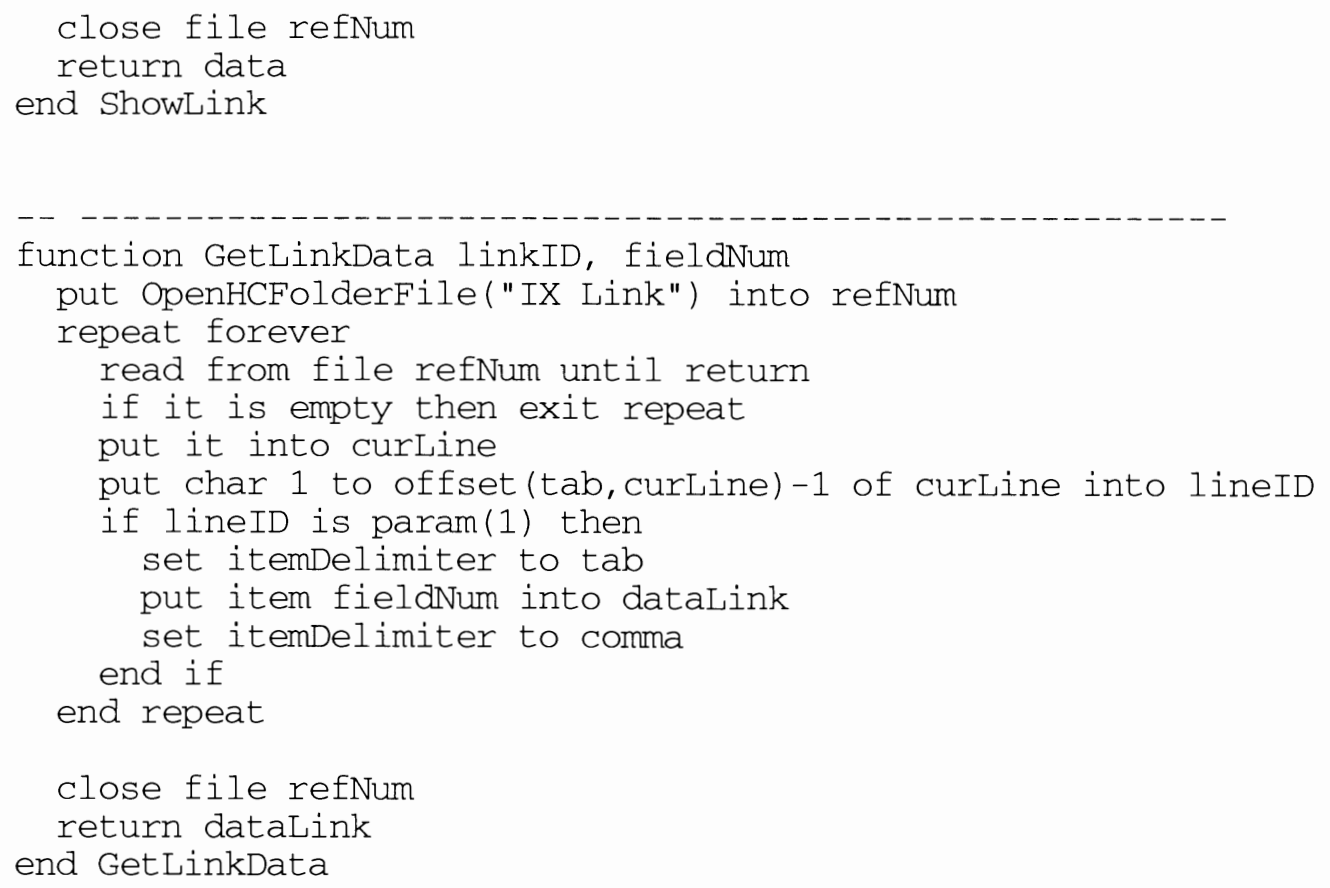

function ModifyLinkstatus linkID, statusMsg

if linkID is not empty and statusMsg is not empty then put empty into data put OpenHCFolderFile("IX Link") into refNum

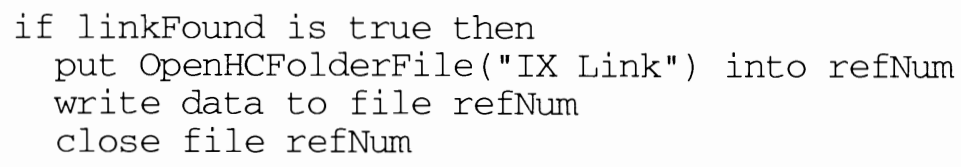




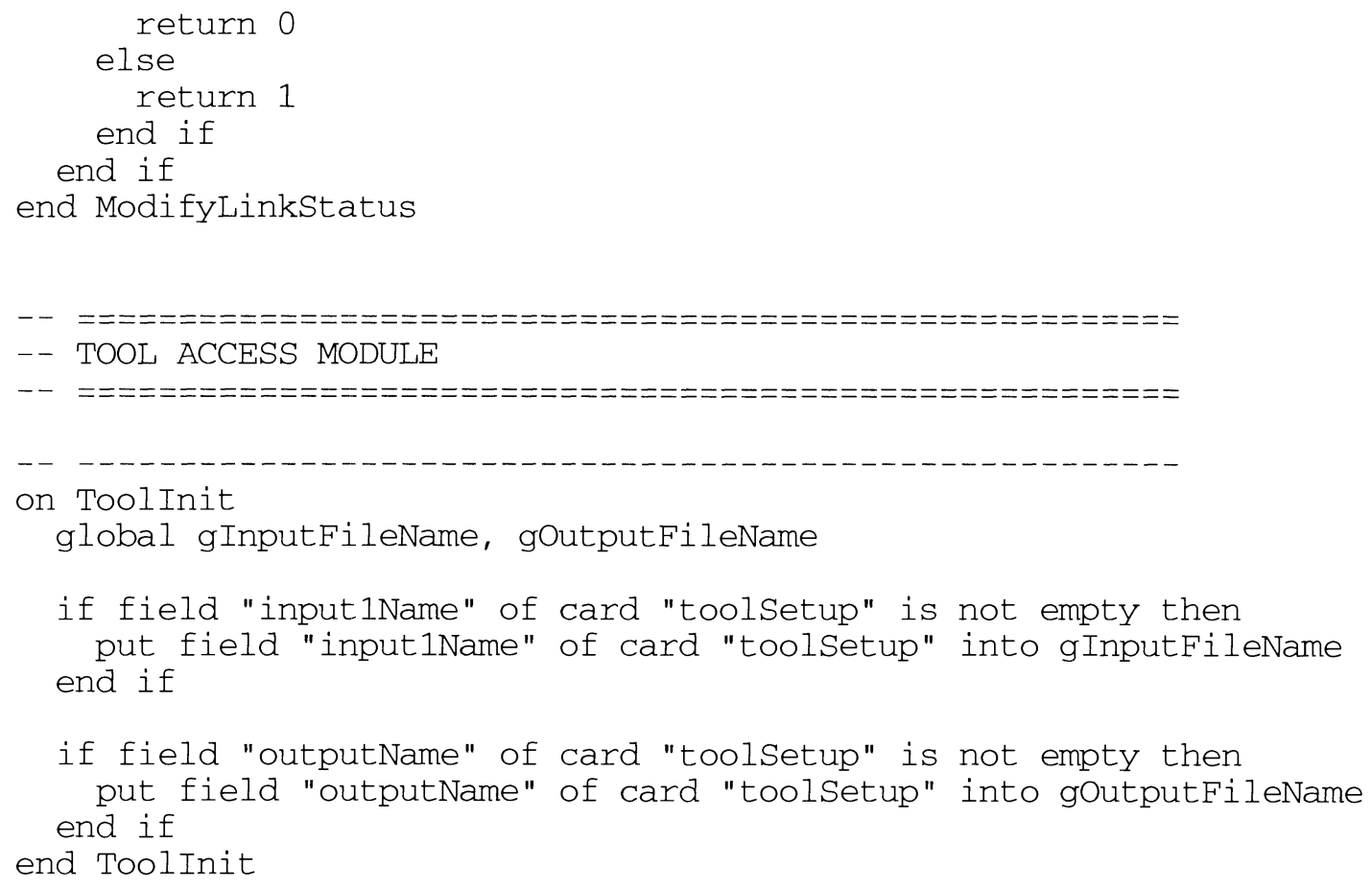

on NewMiscTooldata fileName

put fileName \& "_M" into fileName

open file filename

put numTochar(29) after data

write empty to file fileName

close file fileName

end NewMiscToolData

function ReadMiscToolData fileName, num

put empty into data

put 1 into blockCnt

put fileName \& "_M" into fileName

open file filename

repeat

read from file fileName until numToChar(29)

if it is empty or it is eof then exit repeat

if num is blockCnt then 
delete last char of it put it into data exit repeat

end if

add 1 to blockCnt

end repeat

close file fileName

return data

end ReadMiscToolData

- - UTILITIES

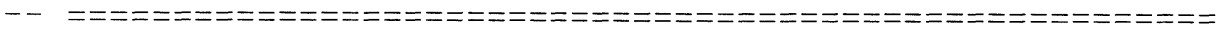

on SysMessage msgText, showAlert

put the short date \&\& the long time \&\& ":" \&\& tab \& msgText \& return after $\neg$

field "newMsgList" of card "messages"

if showAlert is true then

answer "System-Message: " \& return \& msgText with "OK" end if

show button "messageIcon" of card "main" end SysMessage 


\section{B IX External Command Listings}

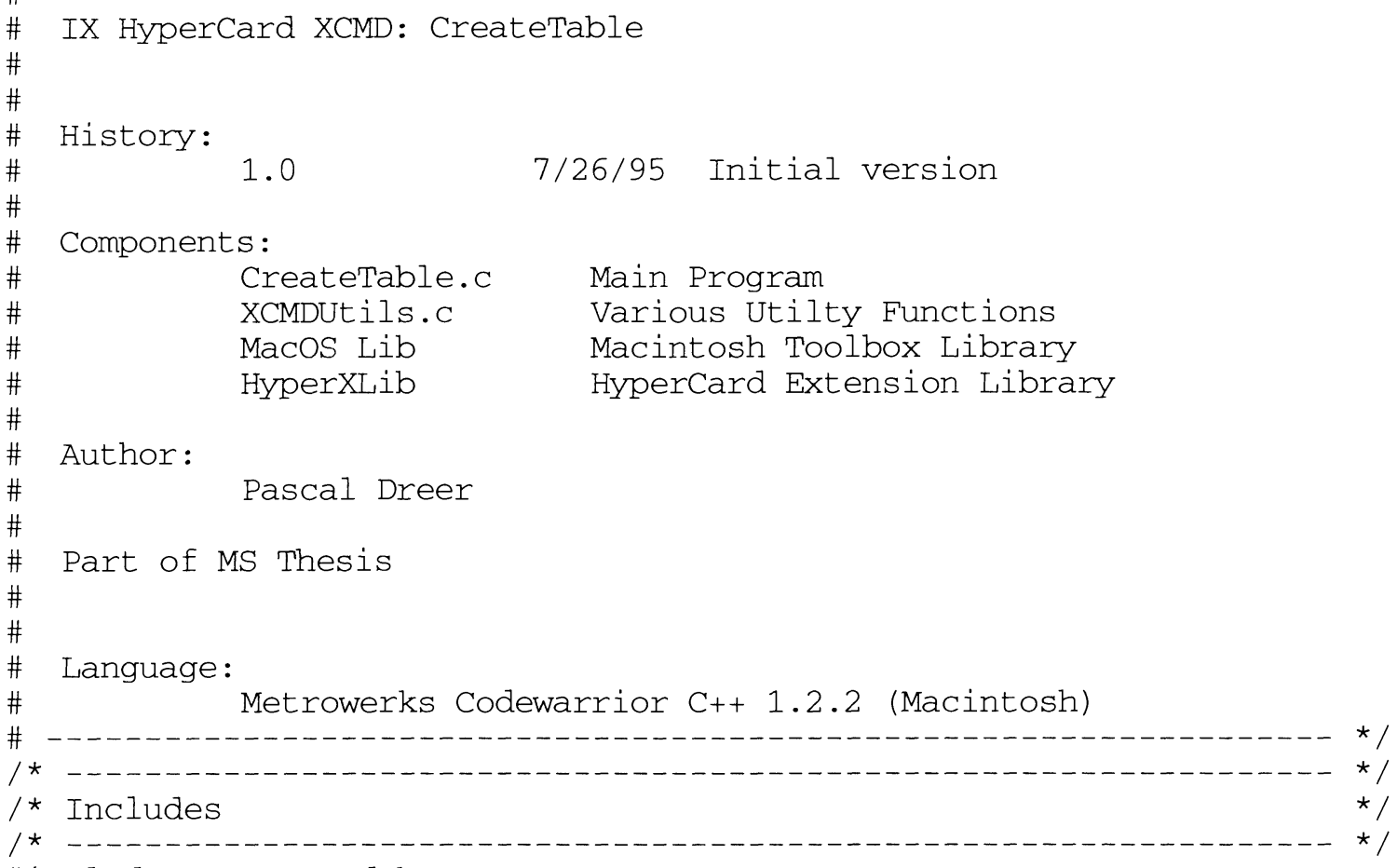

void DoStuff(XCmdPtr paramPtr);

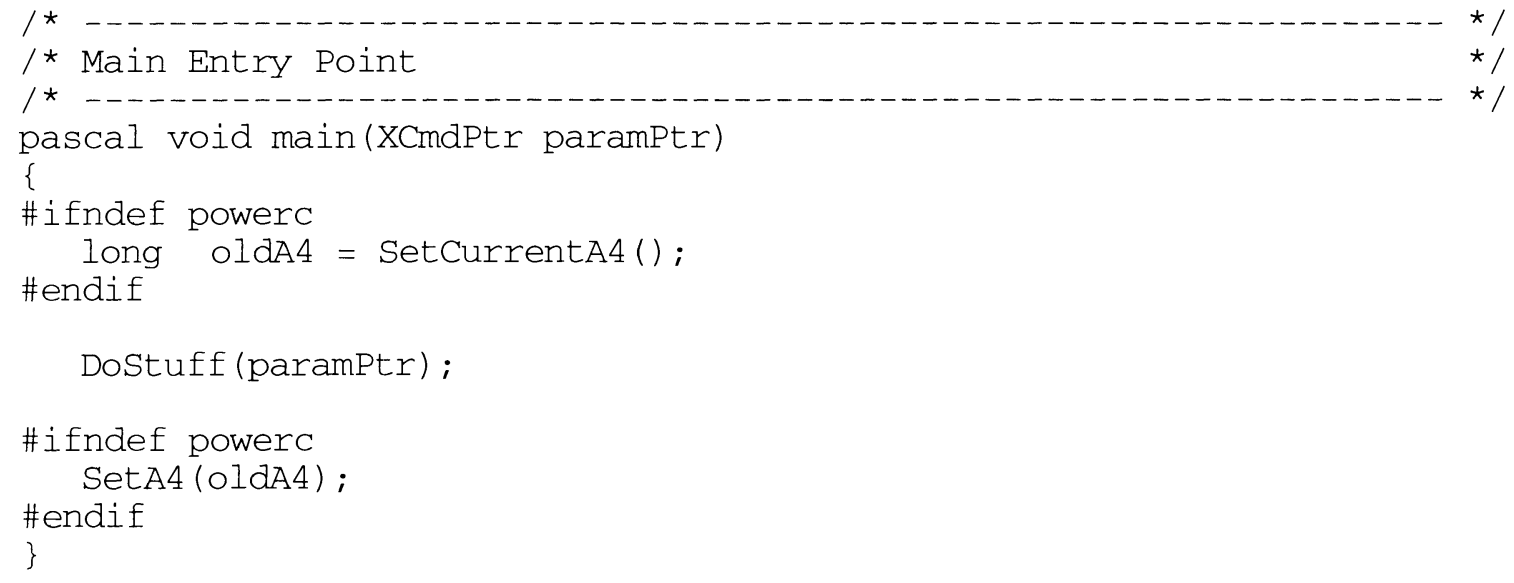




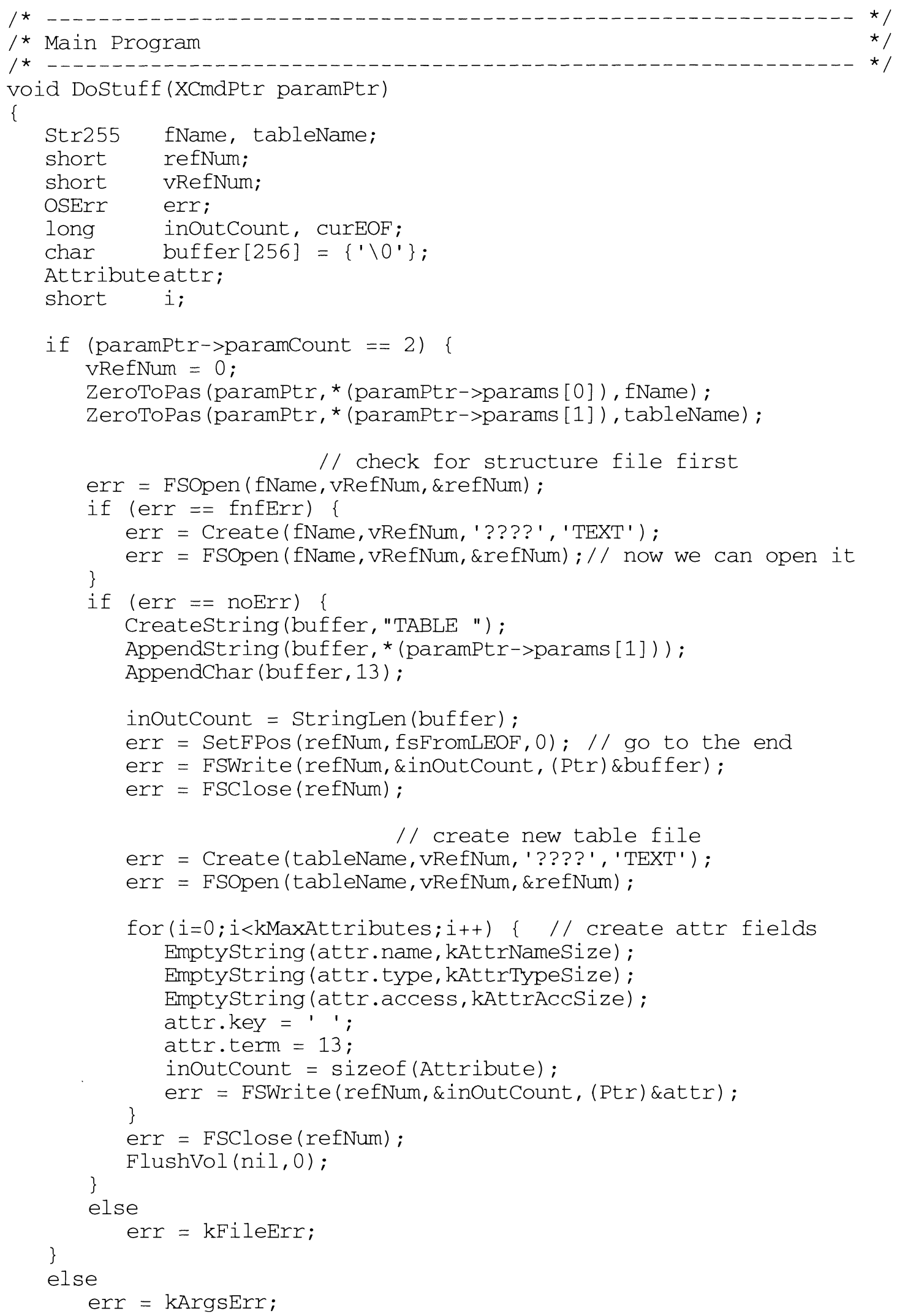




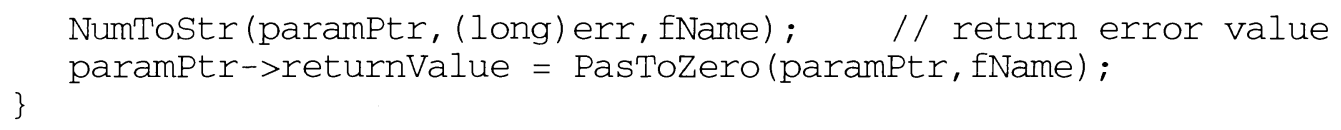




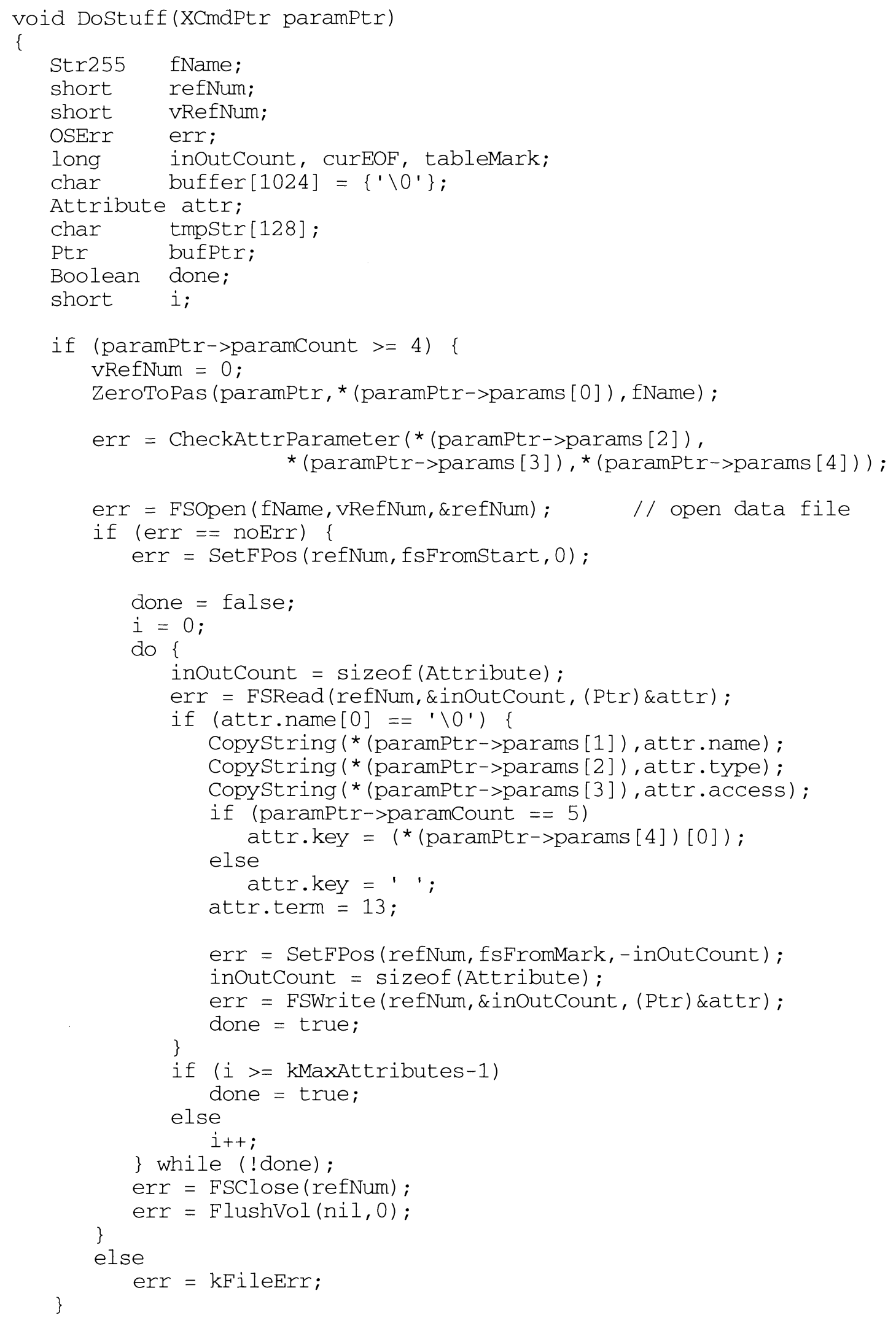




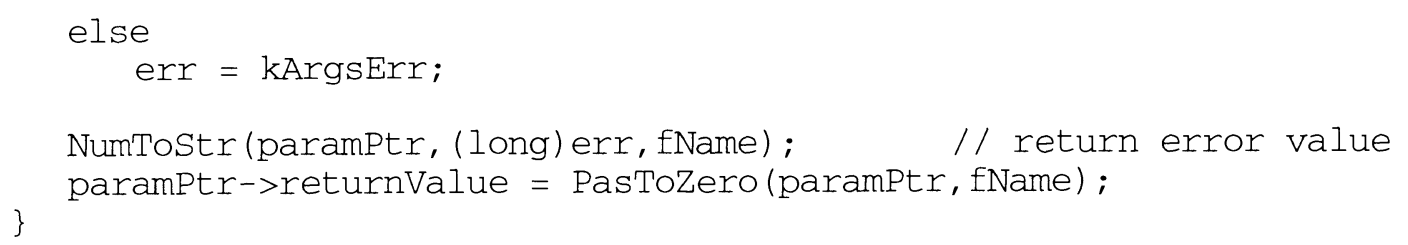




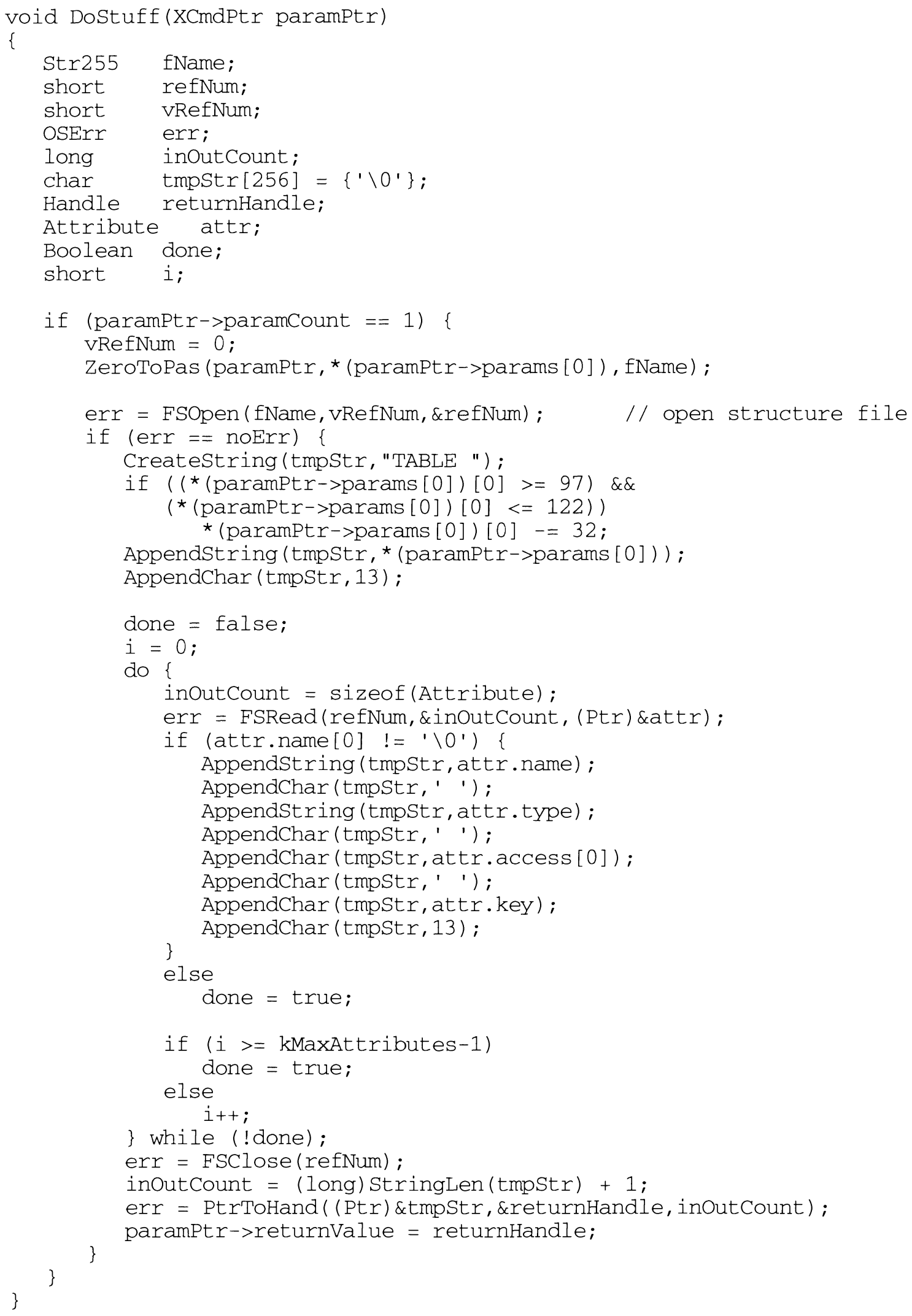


IX HyperCard XCMD: RemoveTable

History:

1.0

$7 / 26 / 95$

Initial version

Components :

$$
\text { RmTable.c Main Program }
$$

XCMDUtils.c Various Utilty Functions

Macos Lib

Macintosh Toolbox Library

HyperXLib

HyperCard Extension Library

Author:

Pascal Dreer

Part of MS Thesis

Language:

Metrowerks Codewarrior $\mathrm{C}++1.2 .2$ (Macintosh)

torowerks Codewarrior C+t 1.2 .2 (Macintosh)

/* Includes

/* -

\#include <HyperXCmd.h>

\#include <A4Stuff.h>

\#include <SetUpA4.h>

\#include "XCMDUtils.h"

void DoStuff (XCmdPtr paramPtr);

pascal void main(XCmaPtr paramPtr)

\{

\#ifndef powerc

long oldA4 = SetCurrentA4 ();

\#endif

DoStuff (paramptr);

\#ifndef powerc

SetA4 (oldA4);

\#endif

\}

void DoStuff(XCmdPtr paramPtr)

\{

Str255 fName, tableName;

short refNum;

short vRefNum;

OSErr err; 


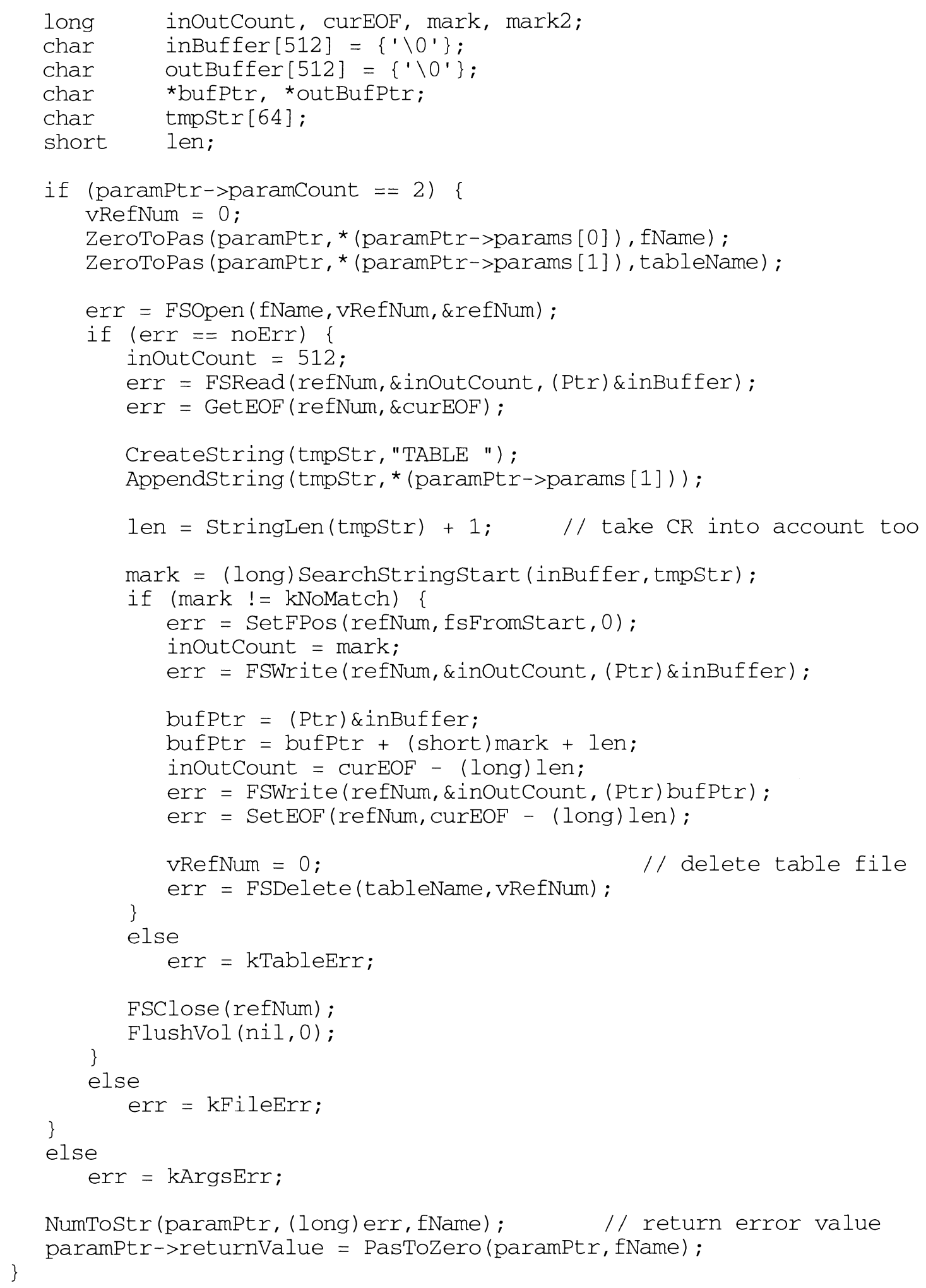


IX HyperCard XCMD: ShowAllTables

History:

$1.0 \quad 7 / 26 / 95 \quad$ Initial version

Components :

ShowAllTable.c Main Program

XCMDUtils.c Various Utilty Functions

MacOS Lib

Macintosh Toolbox Library

HyperXLib

HyperCard Extension Library

Author:

Pascal Dreer

Part of MS Thesis

Language:

Metrowerks Codewarrior $\mathrm{C}++1.2 .2$ (Macintosh)
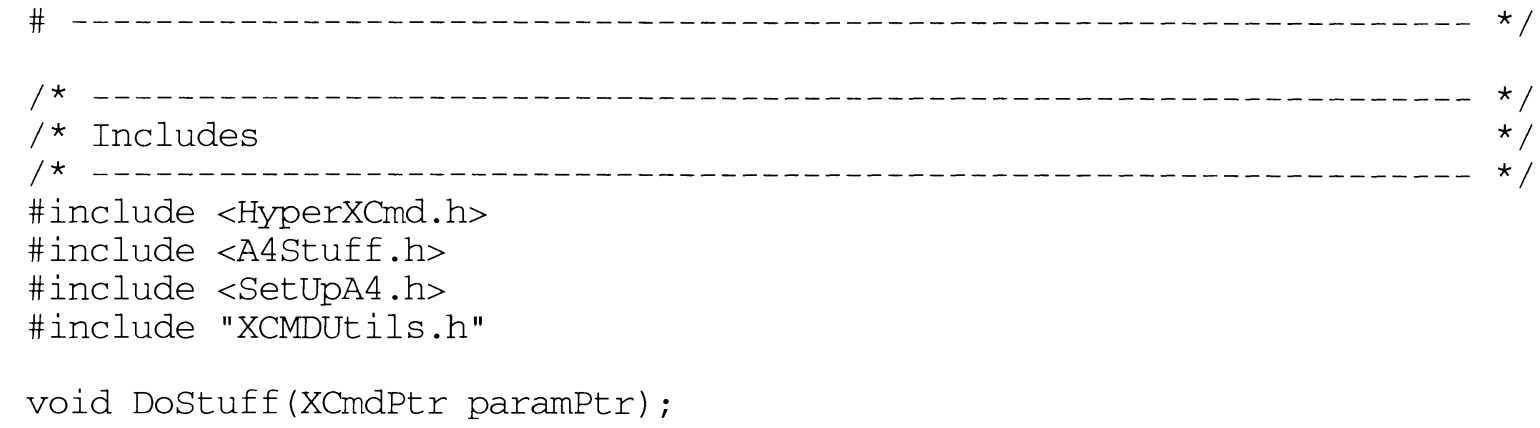

pascal void main (XCmdPtr paramPtr)

\{

\#ifndef powerc

long oldA4 = SetCurrentA4();

\#endif

DoStuff (paramPtr) ;

\#ifndef powerc

SetA4 (oldA4);

\#endif

\}

void DoStuff(XCmdPtr paramPtr)

\{

Str255 fName;

short refNum;

short vRefNum;

OSErr err; 


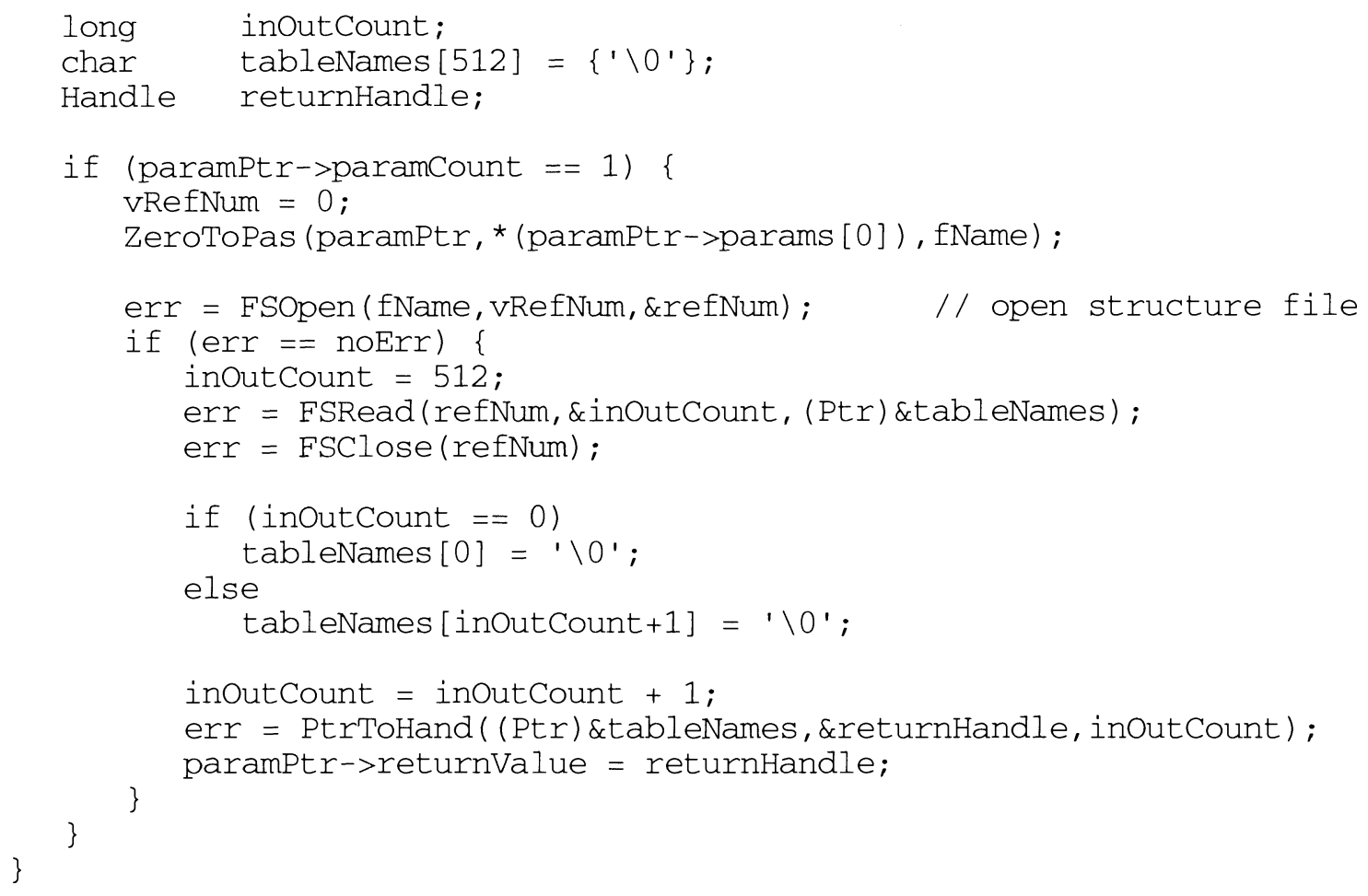




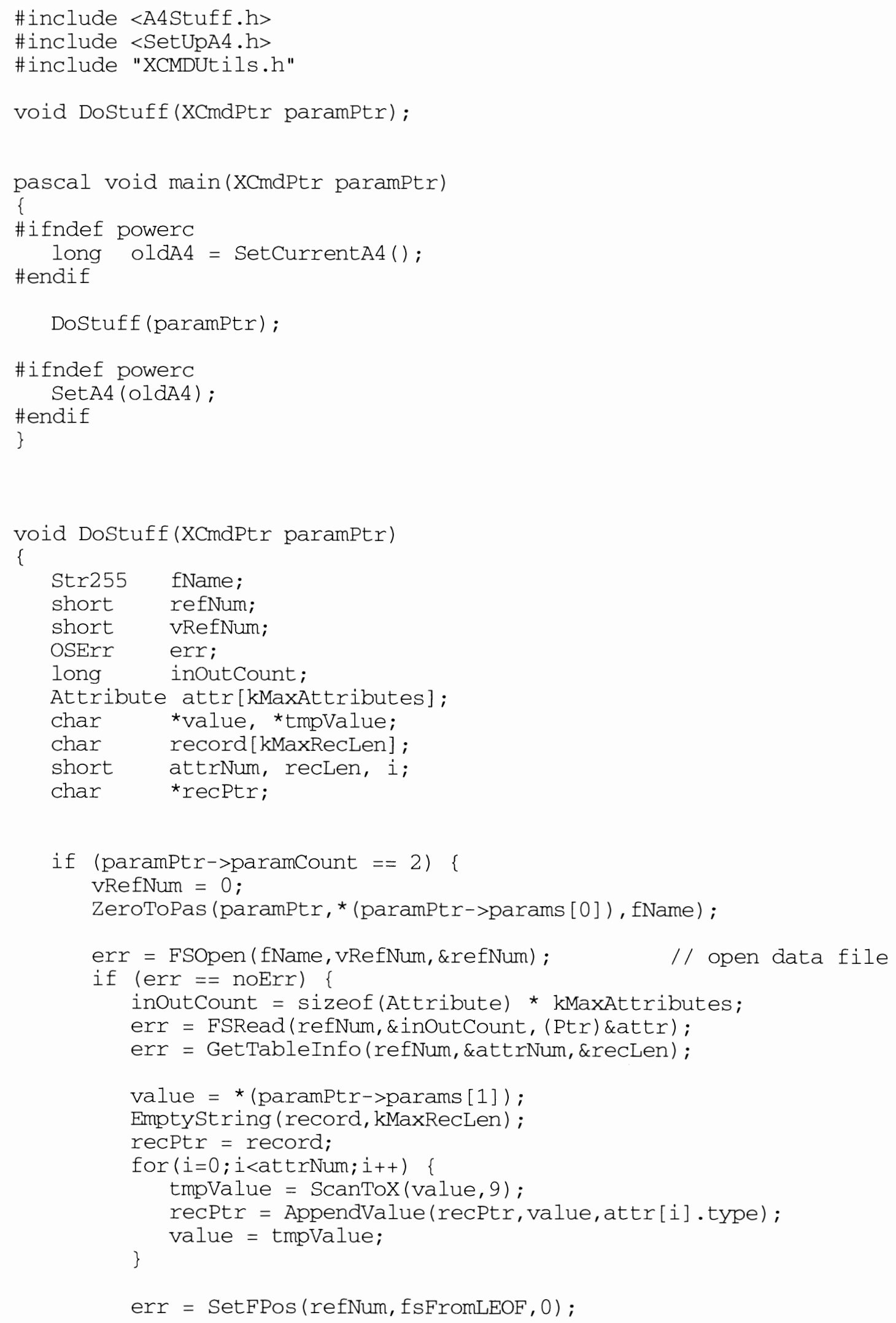




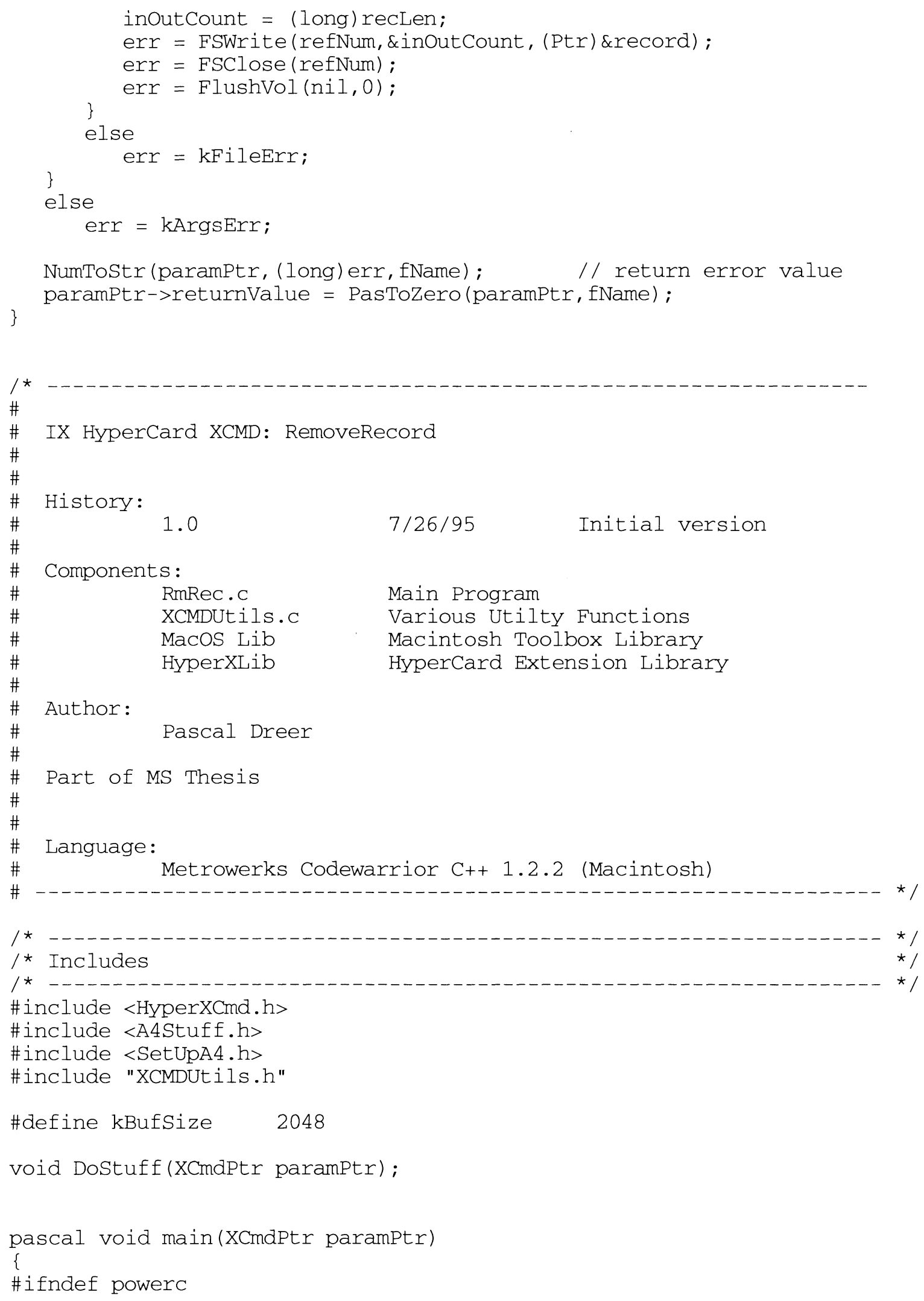




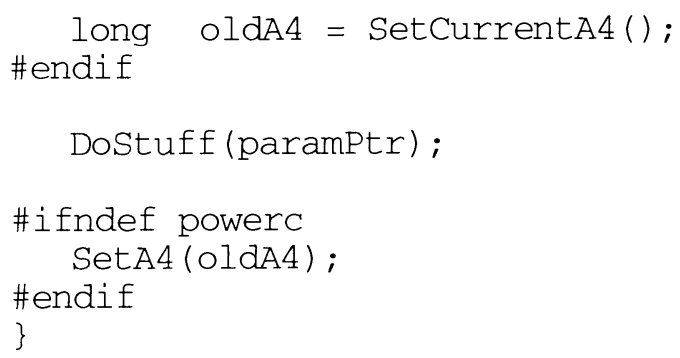




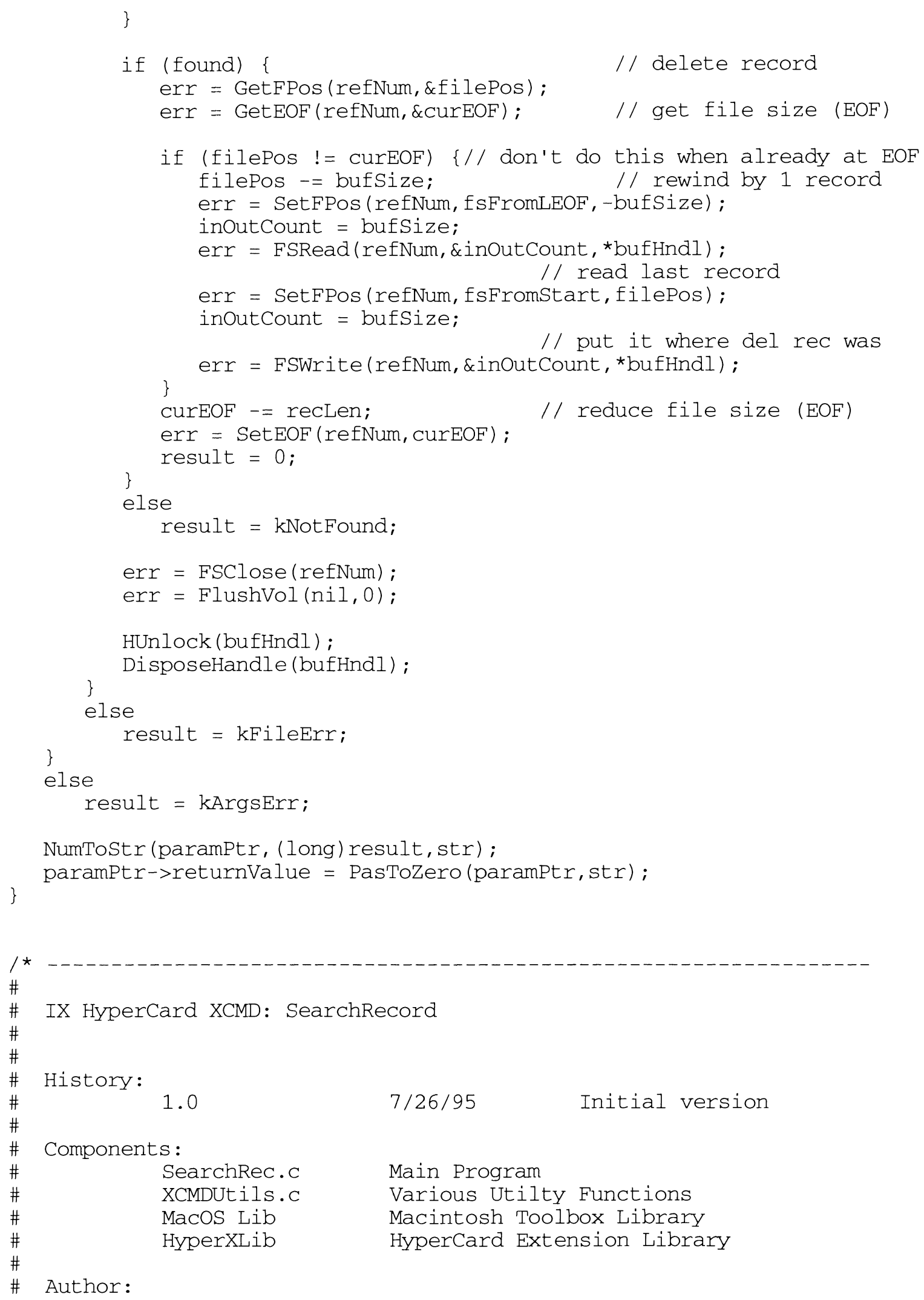




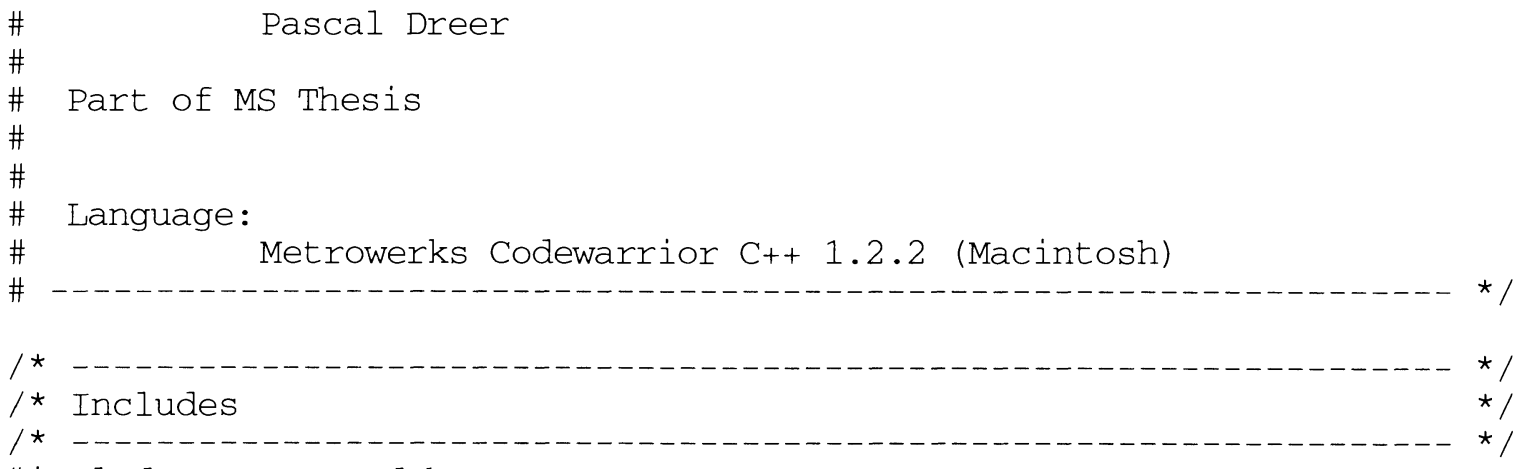

\#include <HyperXCma.h>

\#include <A4Stuff.h>

\#include <SetUpA4.h>

\#include "XCMDUtils.h"

\#define kBufSize $\quad 4096$

\#define kLineSize 1024

\#define kTab 9

void DoStuff(XCmdPtr paramPtr);

pascal void main (XCmdPtr paramPtr)

\{

\#ifndef powerc

long oldA4 = SetCurrentA4();

\#endif

DoStuff (paramPtr) ;

\#ifndef powerc

SetA4 (oldA4);

\#endif

\}

void DoStuff(XCmaPtr paramPtr)

\{

$\begin{array}{ll}\text { Str255 } & \text { fName; } \\ \text { short } & \text { refNum; } \\ \text { short } & \text { vRefNum; } \\ \text { OSErr } & \text { err; } \\ \text { long } & \text { inOutCount; } \\ \text { short } & \text { numOfRecords, result; } \\ \text { Handle } & \text { bufHndl; } \\ \text { Boolean } & \text { done; } \\ \text { short } & \text { found = } ; \\ \text { char } & \text { * ScanPtr; } \\ \text { char } & \text { *attr, *value; } \\ \text { short } & \text { i, attroffet; } \\ \text { AttrInfo attrInfo; } \\ \text { short } & \text { attrNum, recLen; } \\ \text { long } & \text { bufSize; }\end{array}$




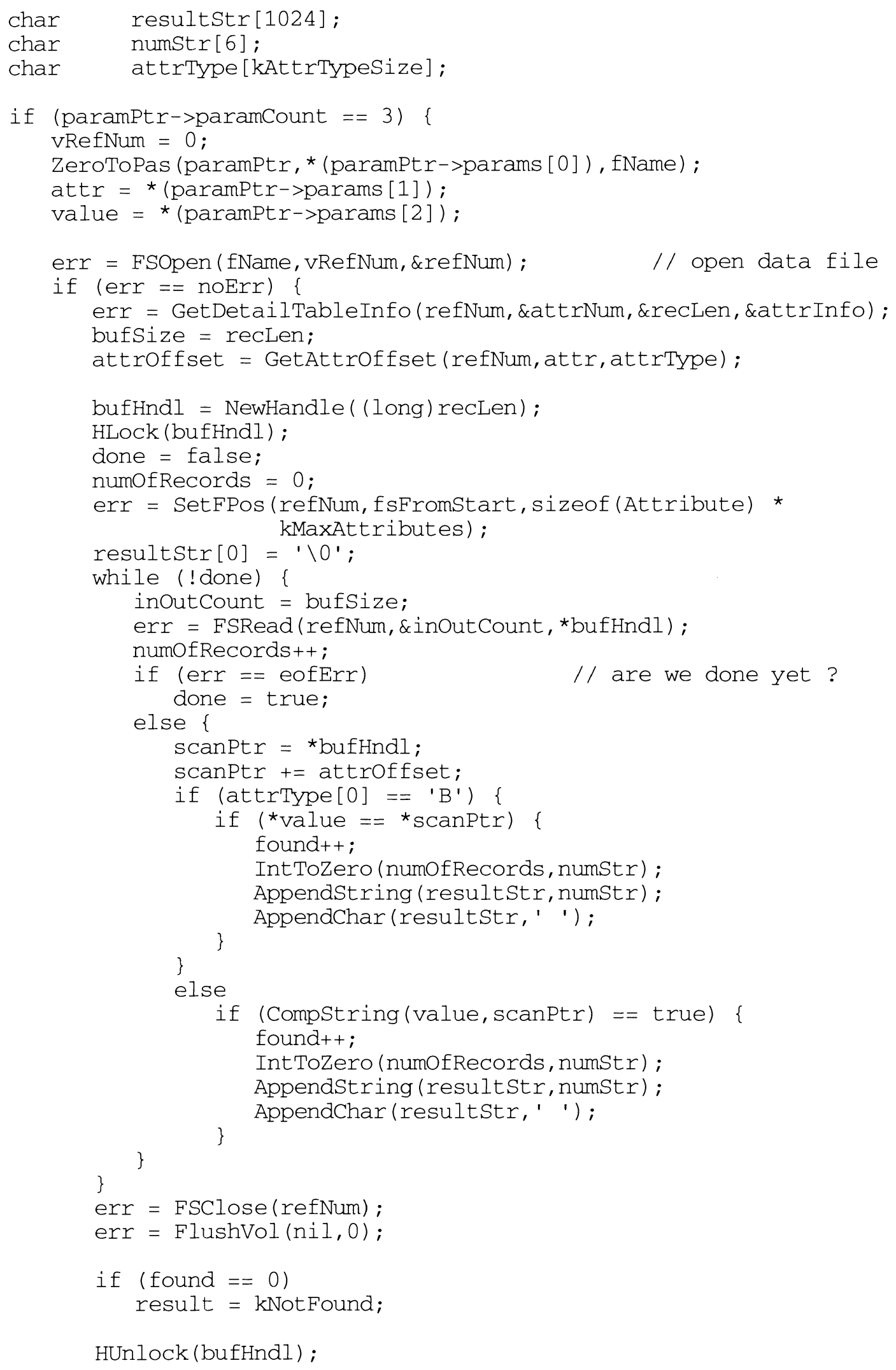

HUnlock (bufHndl) ; 


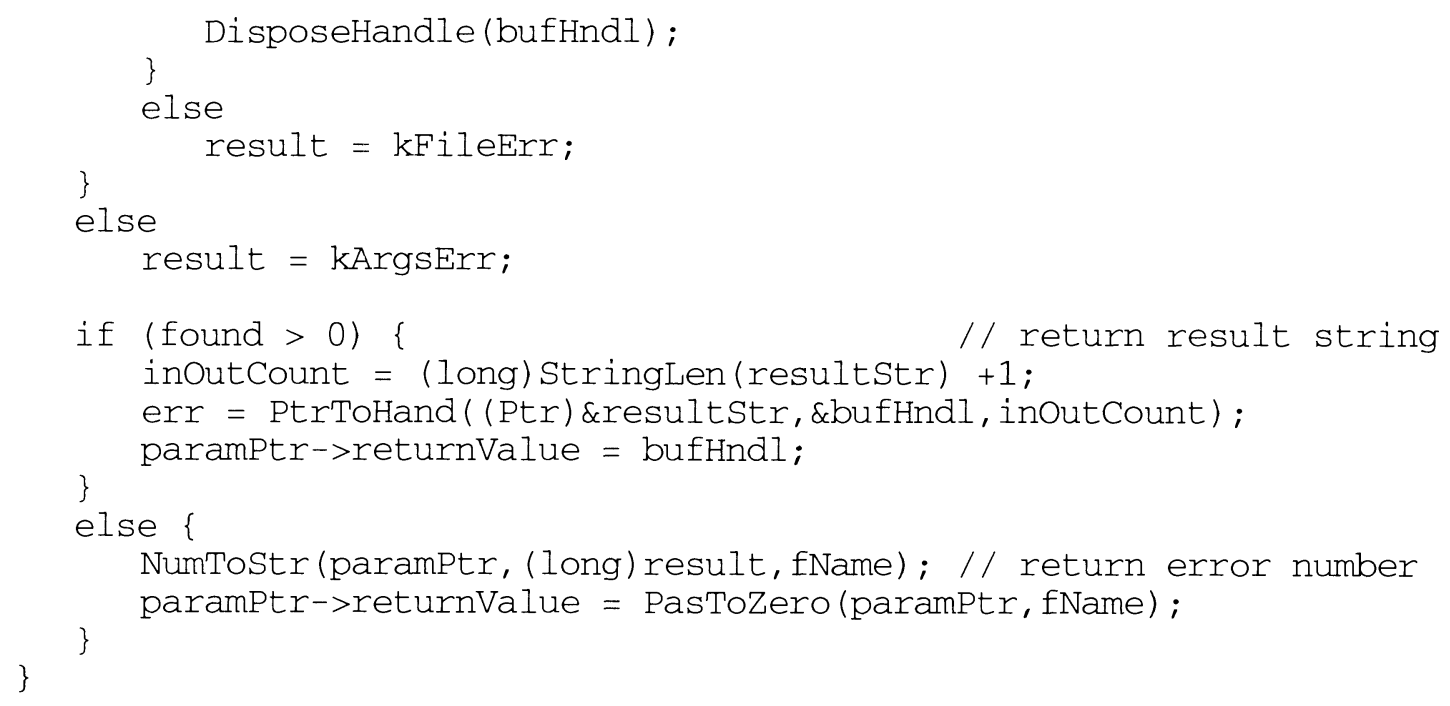


void Dostuff (XCmdPtr paramPtr);

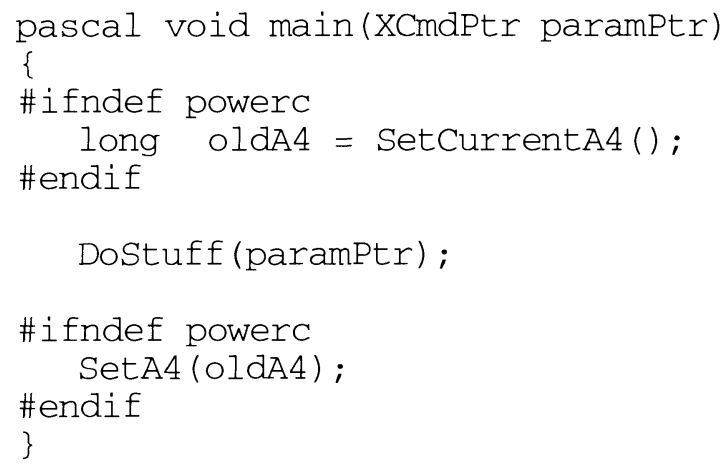




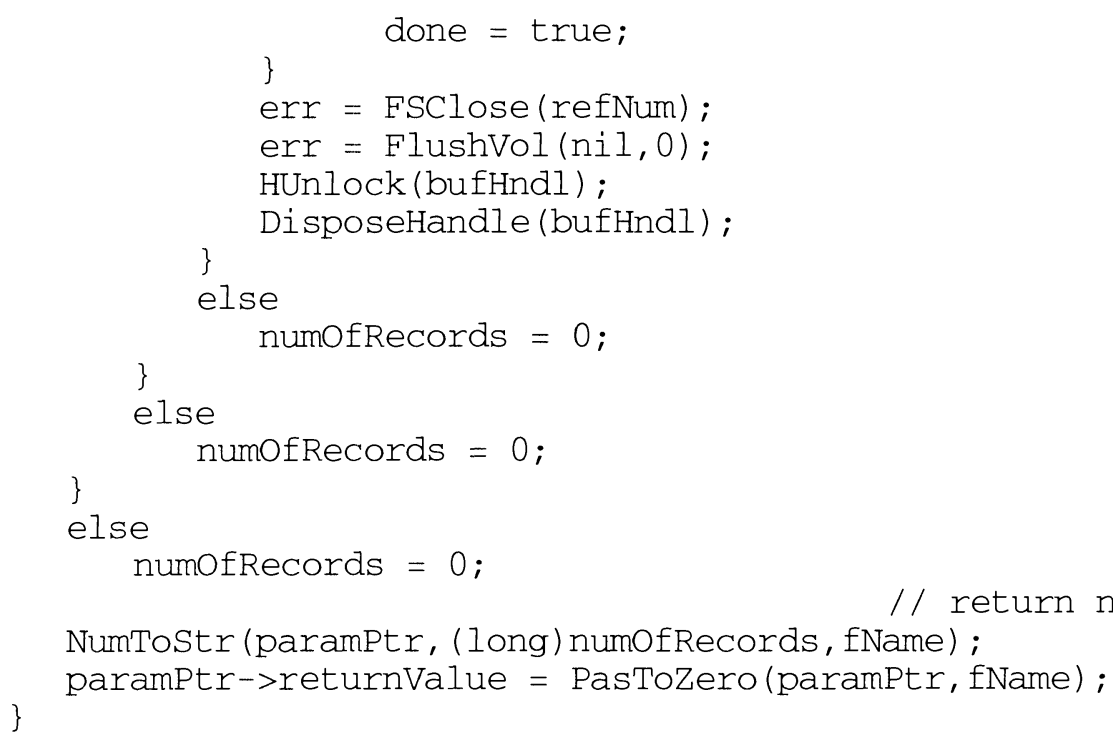

\#define kLineSize 1024 


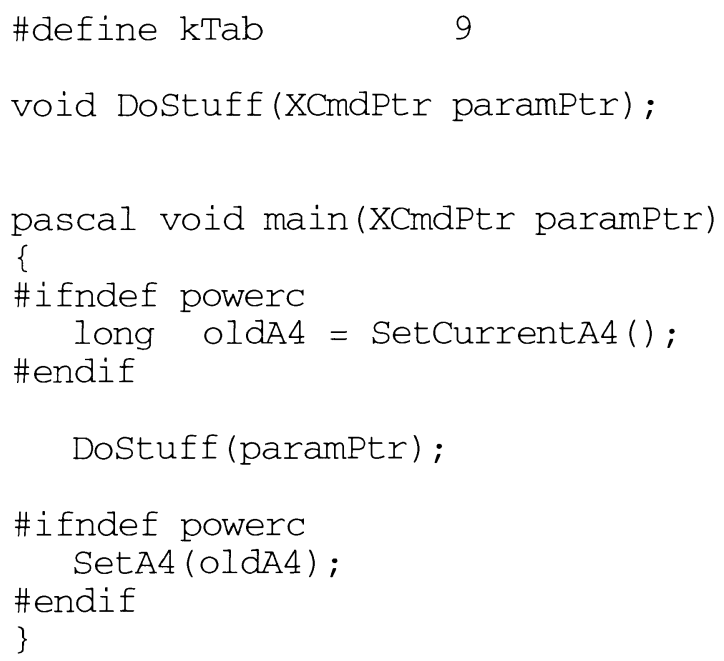




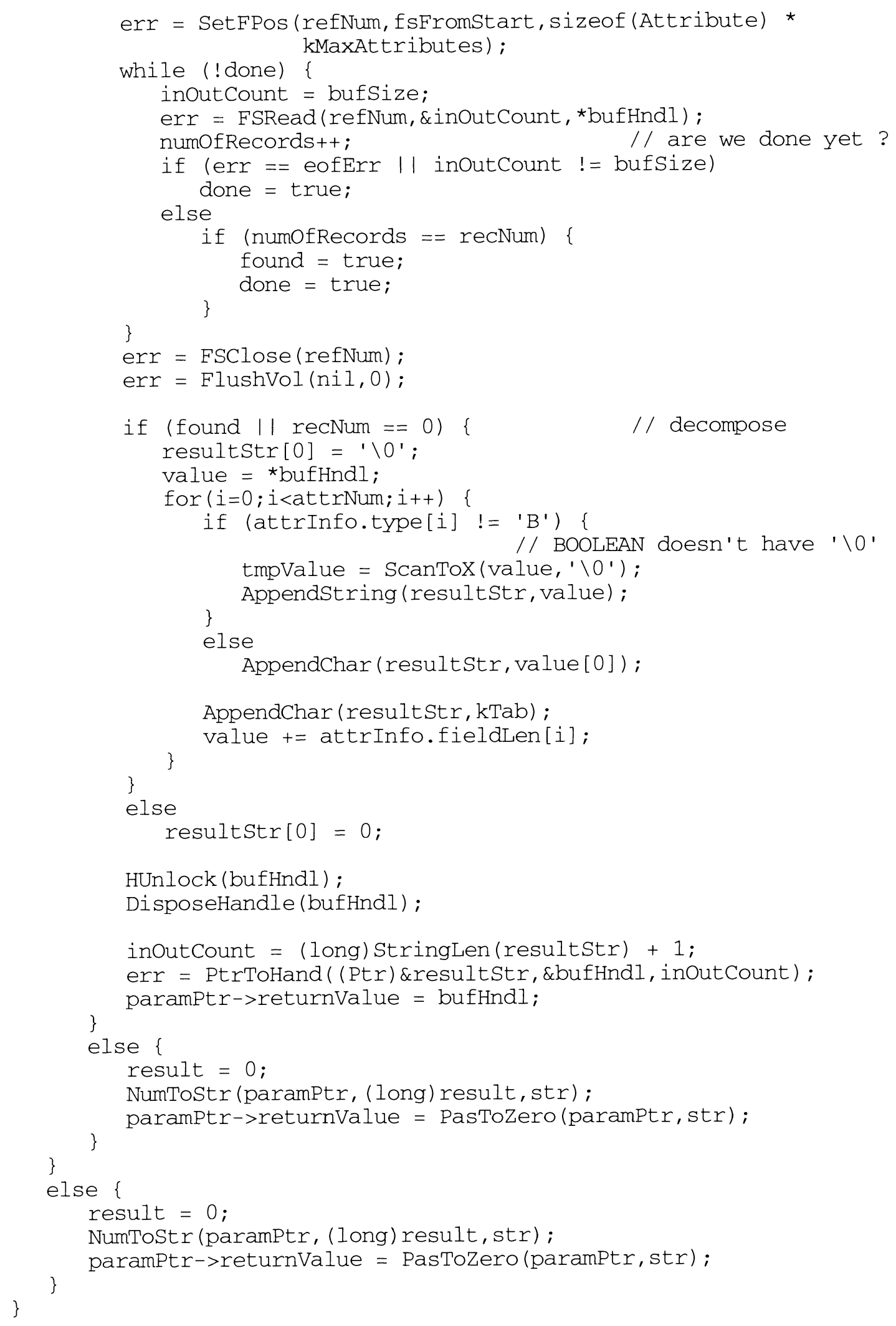


IX HyperCard XCMD: GetRecordValue

History:

$1.0 \quad 7 / 26 / 95$ Initial version

Components :

GetRecVal.c Main Program

XCMDUtils.c Various Utilty Functions

MacOS Lib Macintosh Toolbox Library

HyperXLib HyperCard Extension Library

Author:

Pascal Dreer

Part of MS Thesis

Language:

Metrowerks Codewarrior $\mathrm{C}++1.2 .2$ (Macintosh)

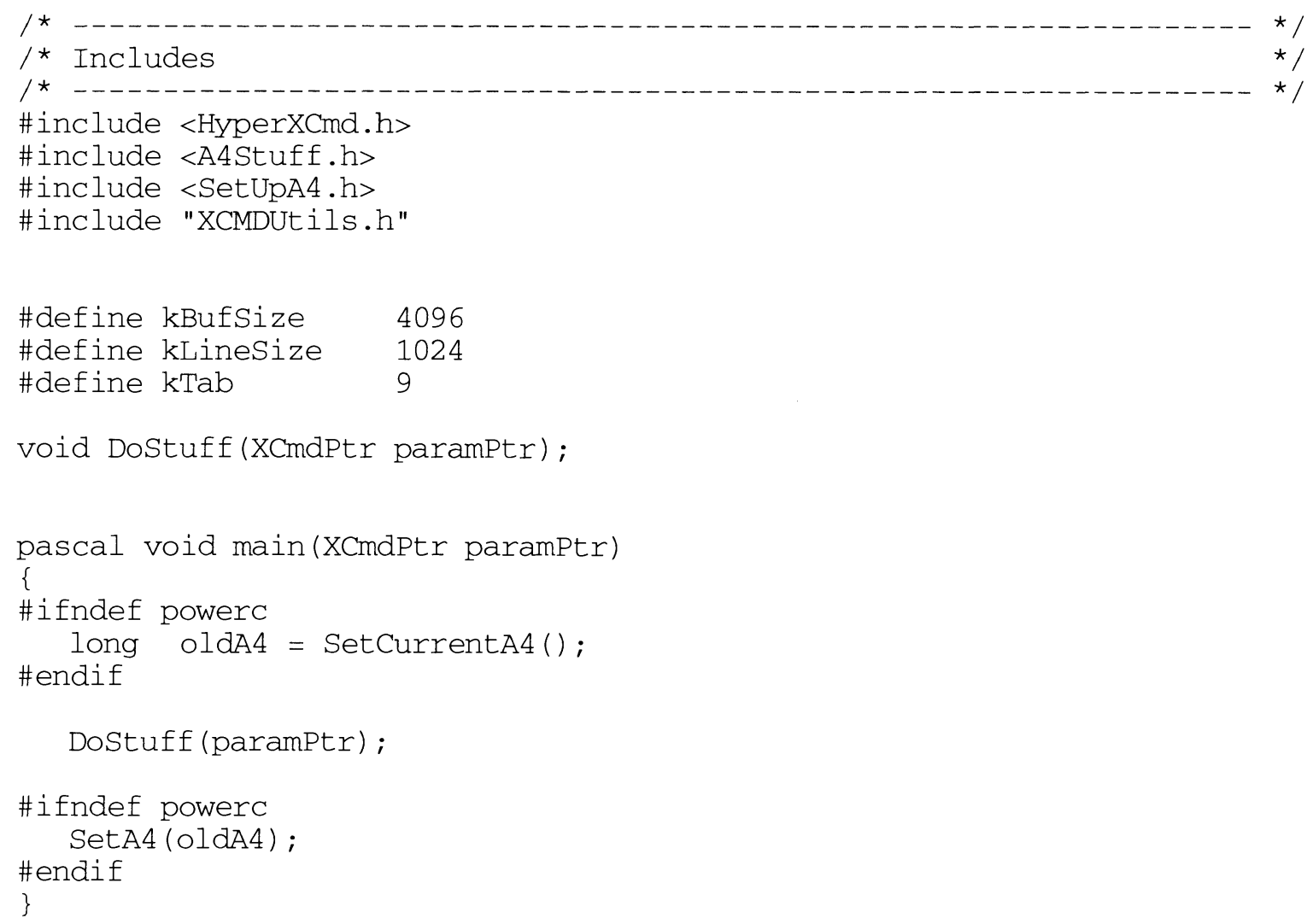




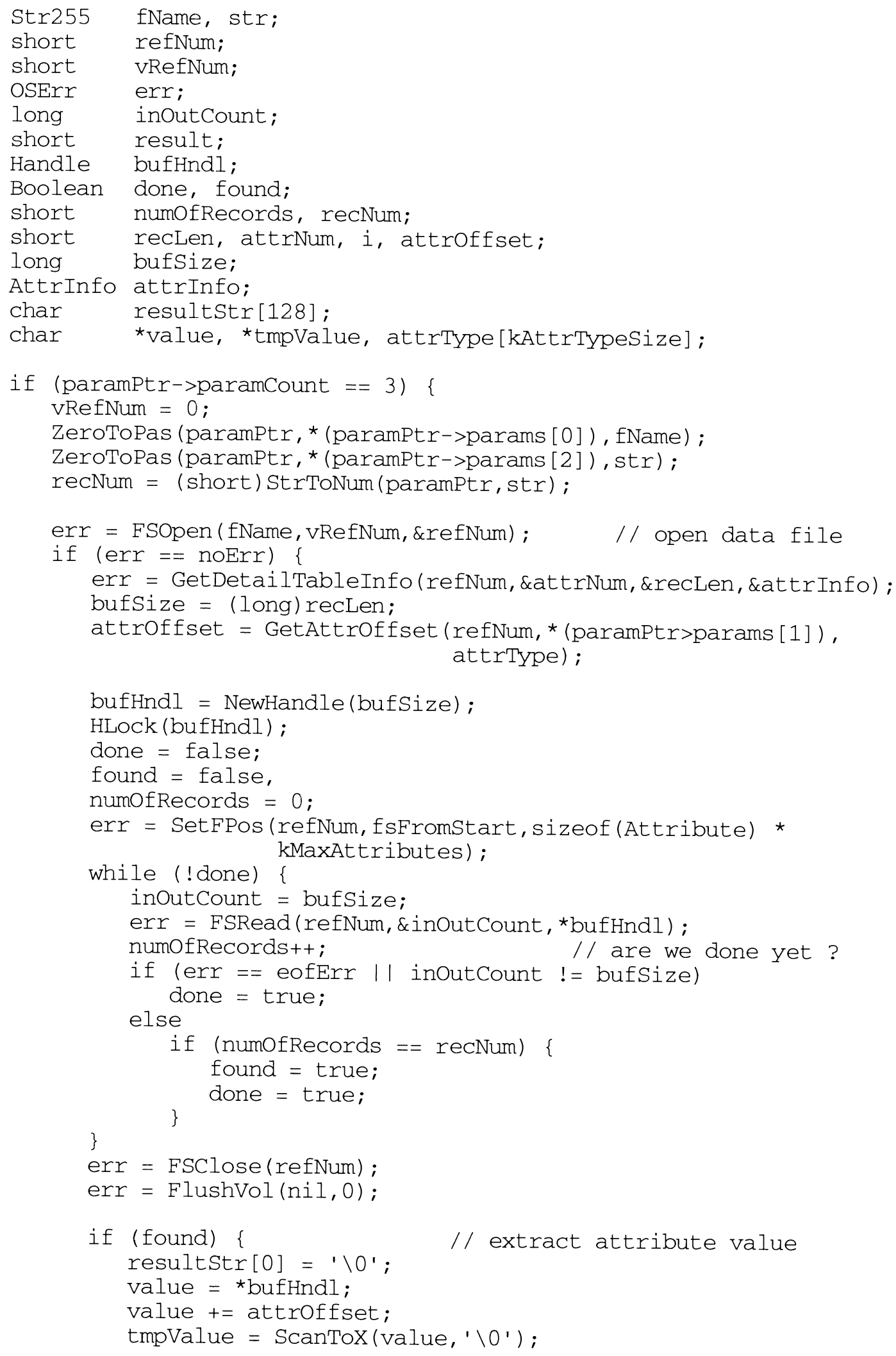




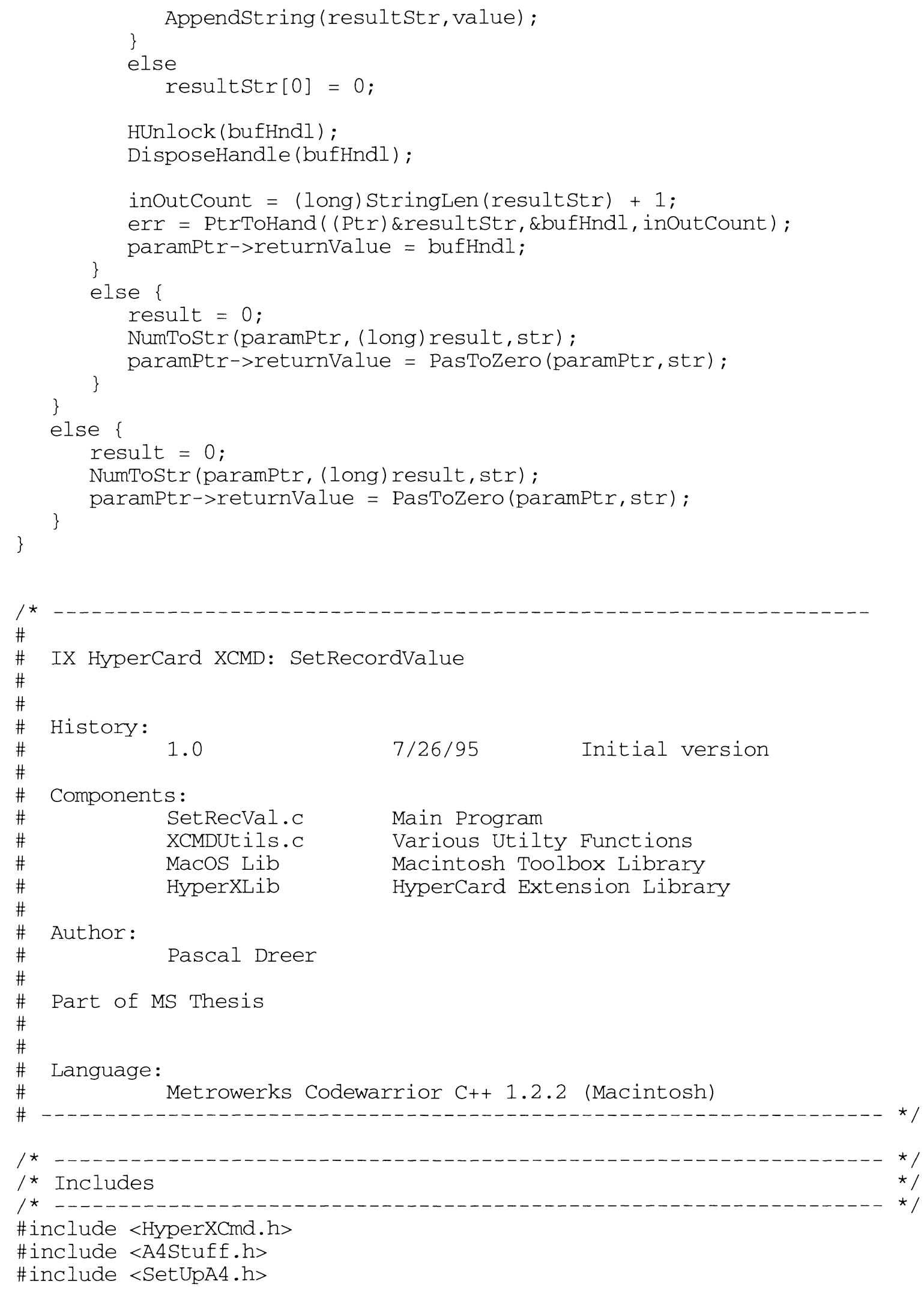




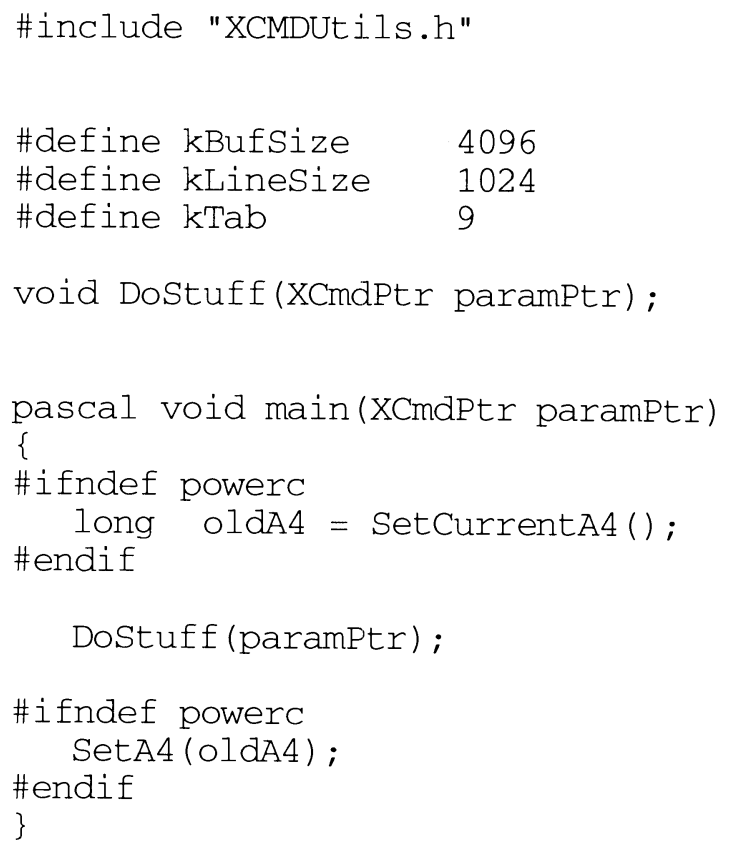




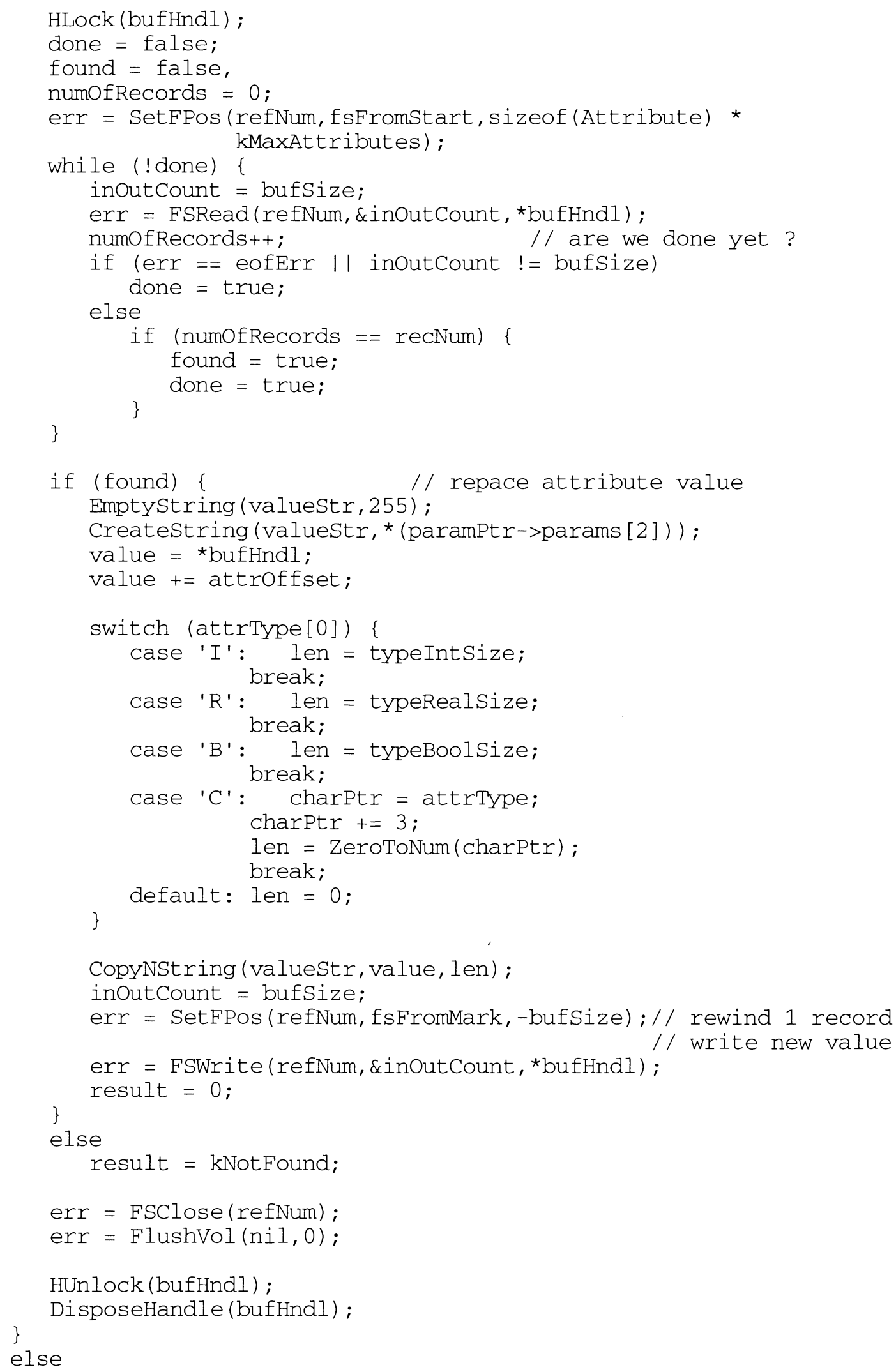




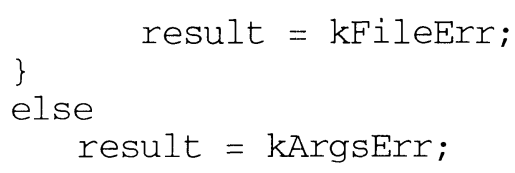




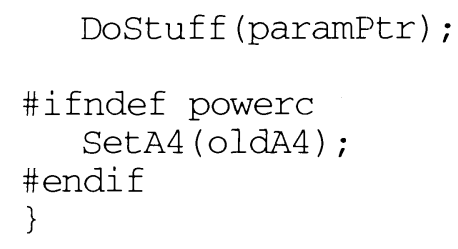




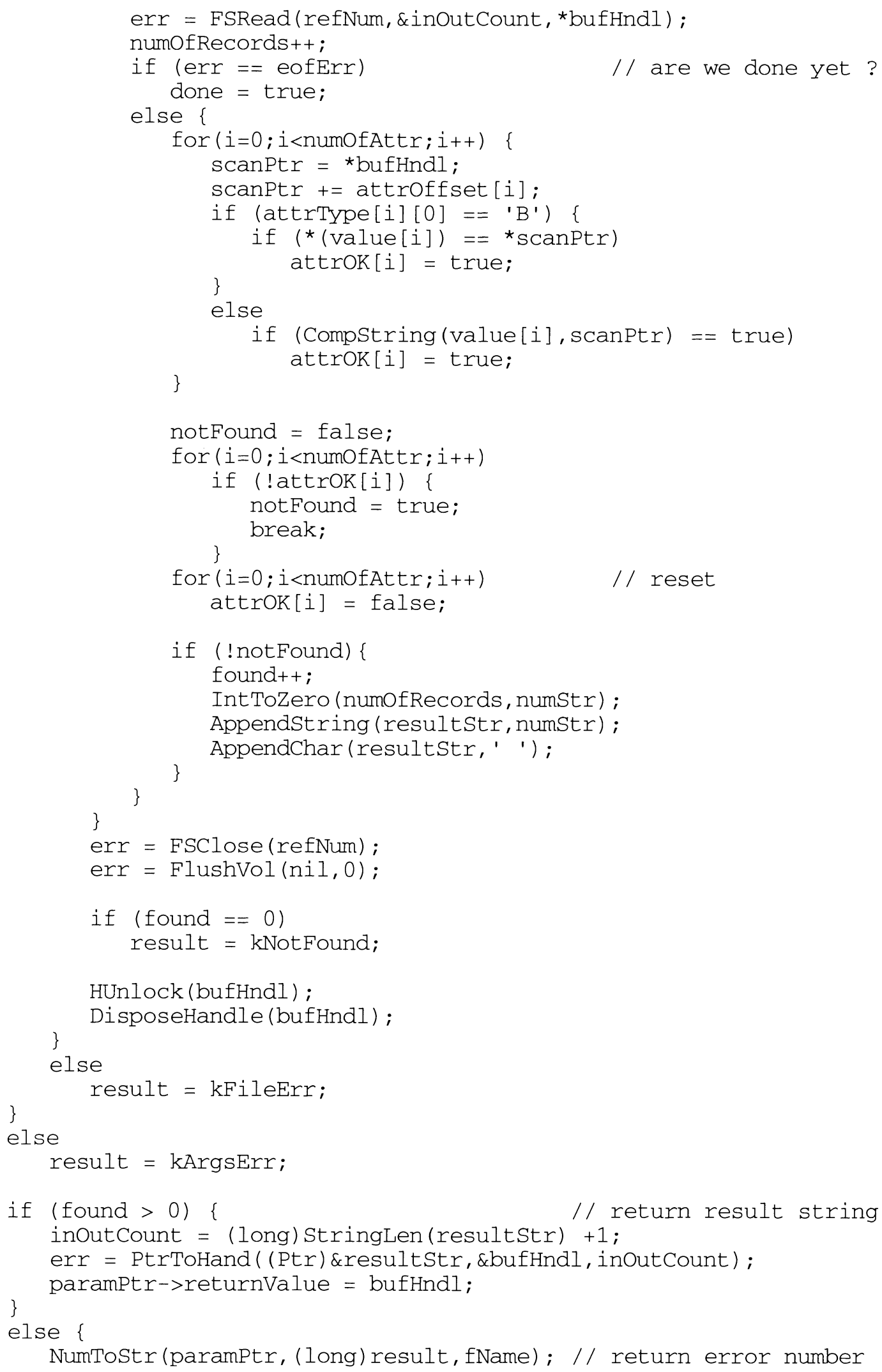




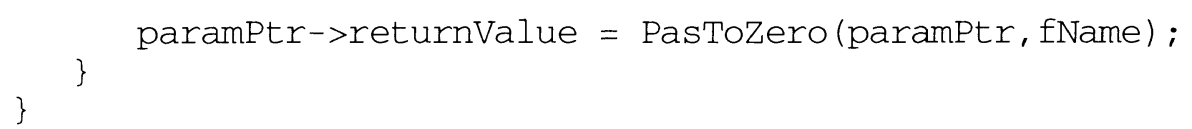


\#endif

\}
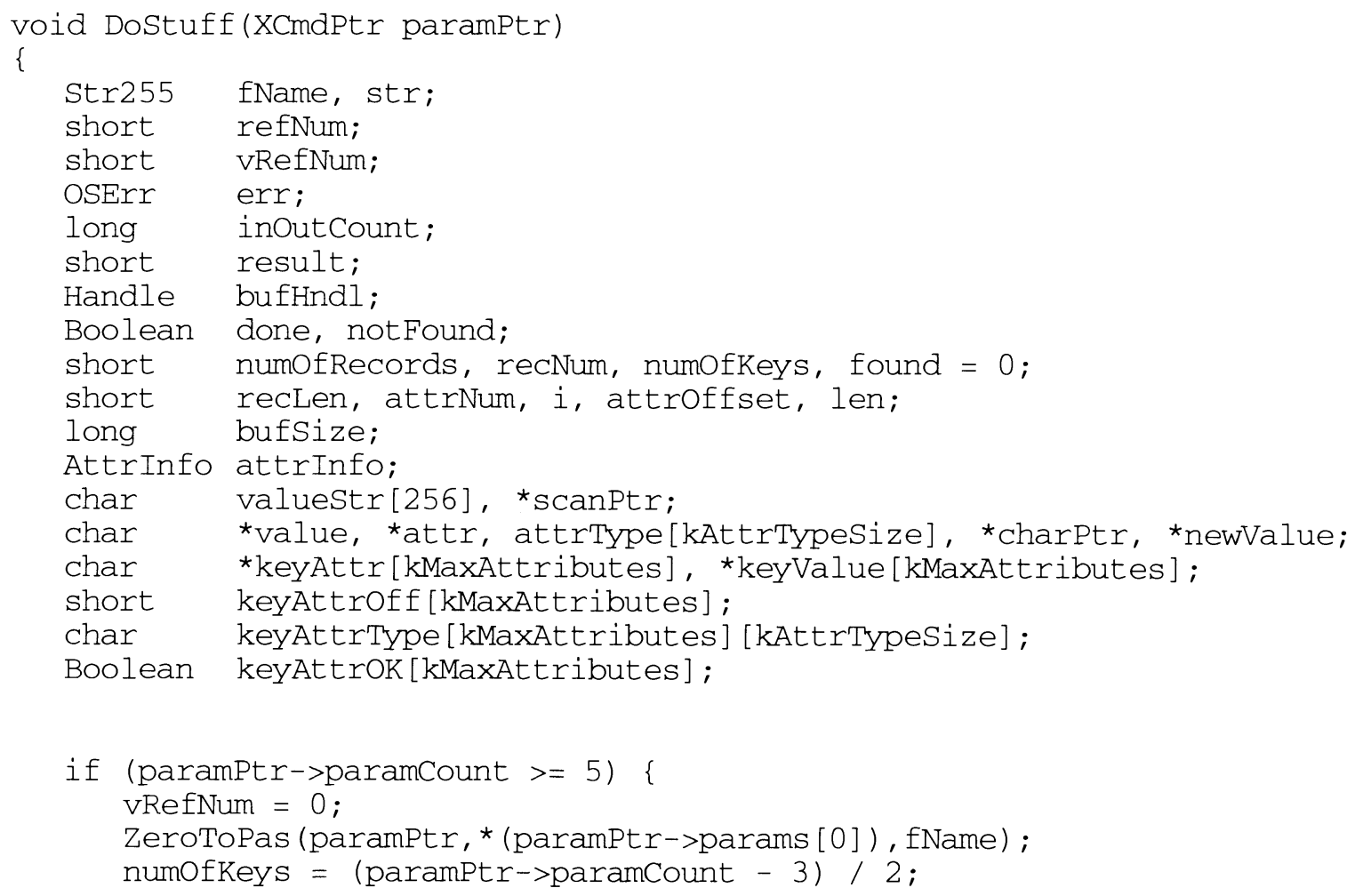


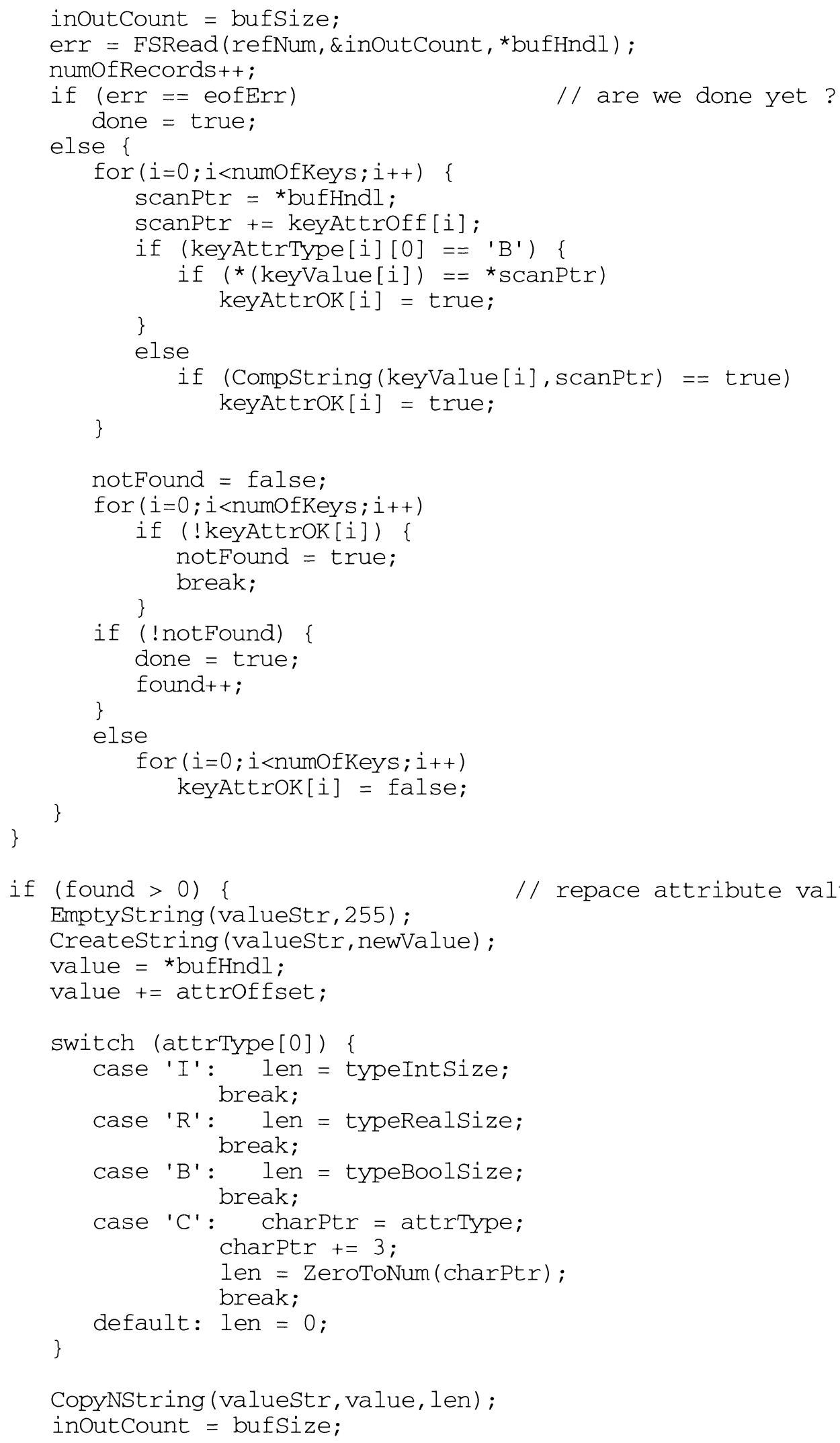




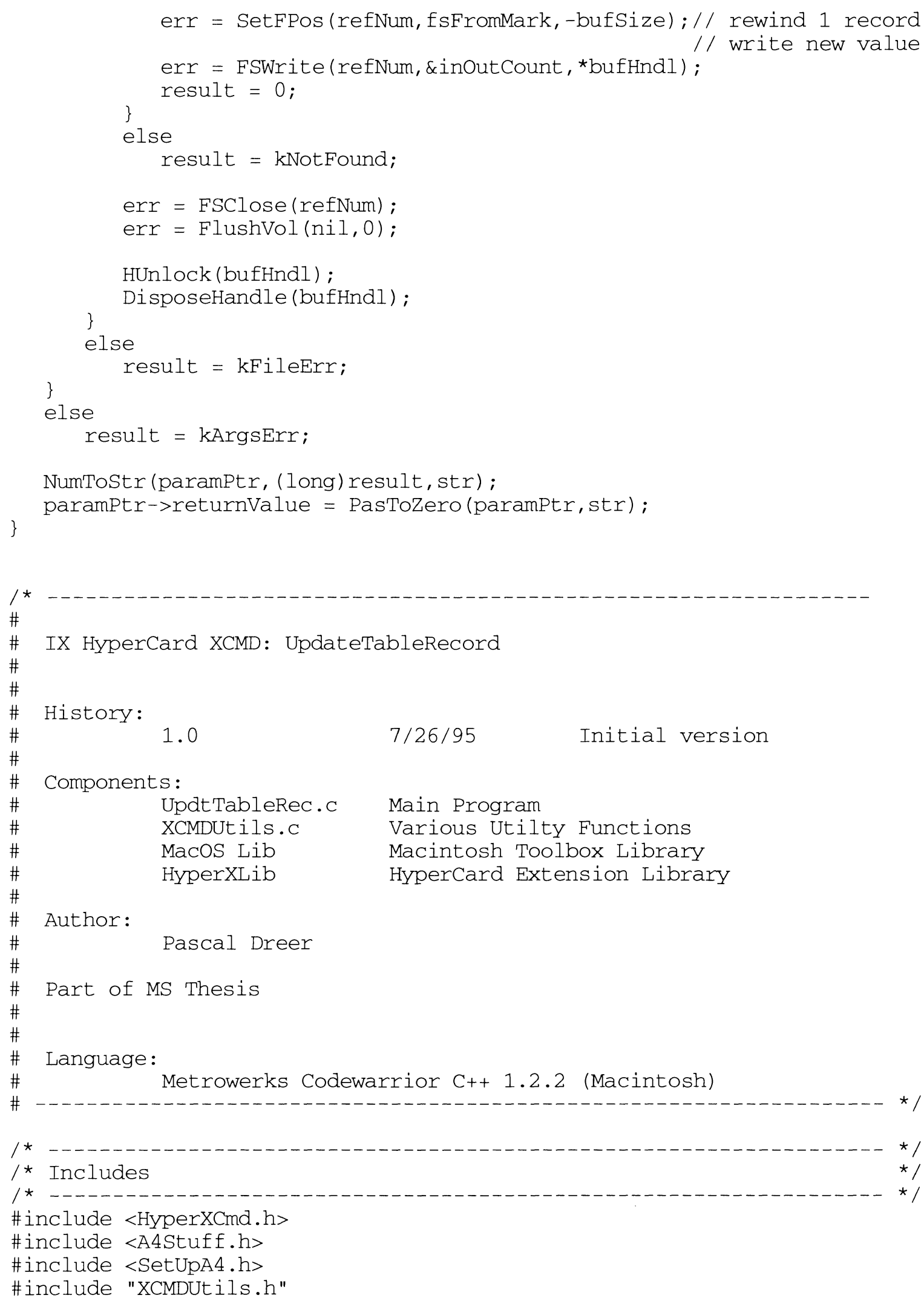


$\begin{array}{ll}\text { \#define kBufSize } & 4096 \\ \text { \#define kLineSize } & 1024 \\ \text { \#define kTab } & 9 \\ & \\ \text { void DoStuff(XCmdPtr } & \text { paramPtr) ; }\end{array}$

pascal void main(XCmaPtr paramPtr)

\{

\#ifndef powerc

long oldA4 = SetCurrentA4();

\#endif

Dostuff (paramPtr) ;

\#ifndef powerc

SetA4 (oldA4);

\#endif

\}

void DoStuff(XCmdPtr paramPtr)

\{

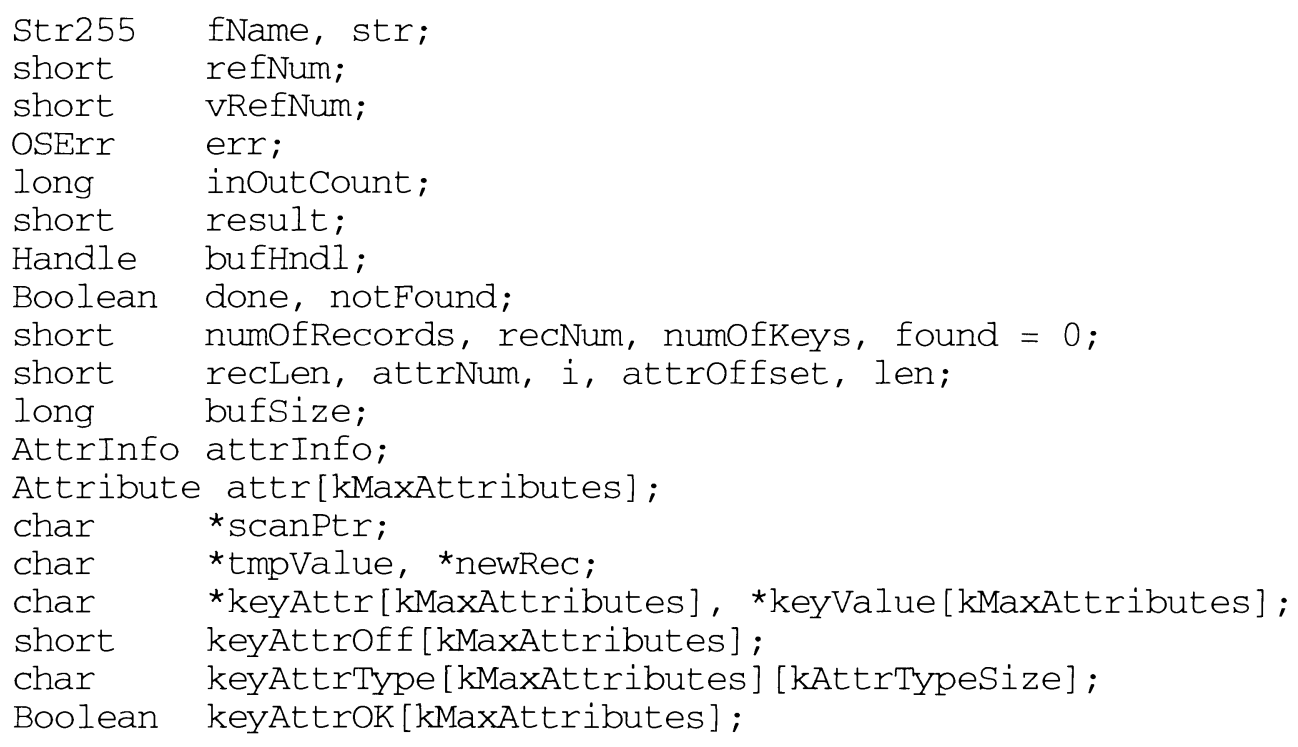




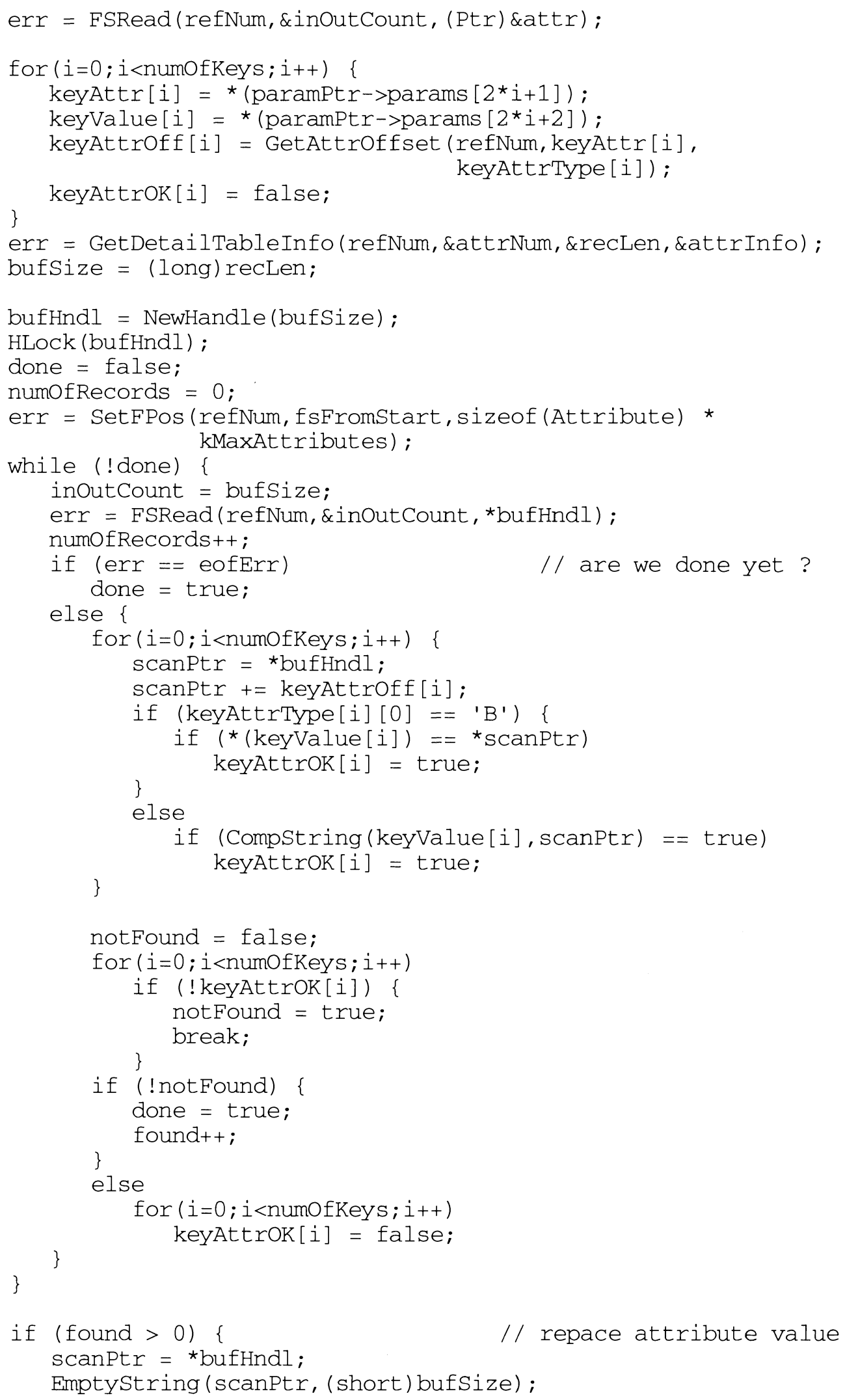




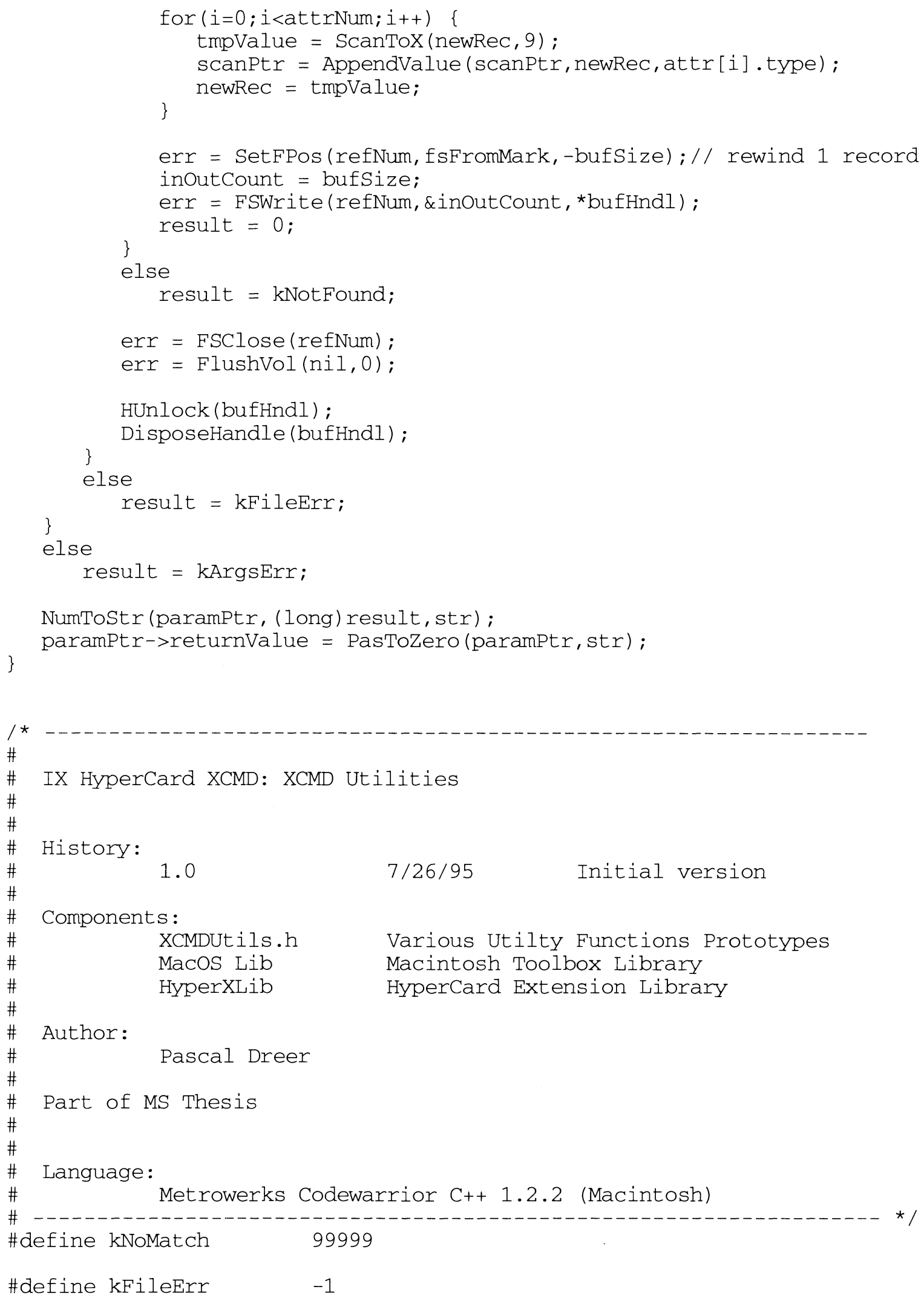




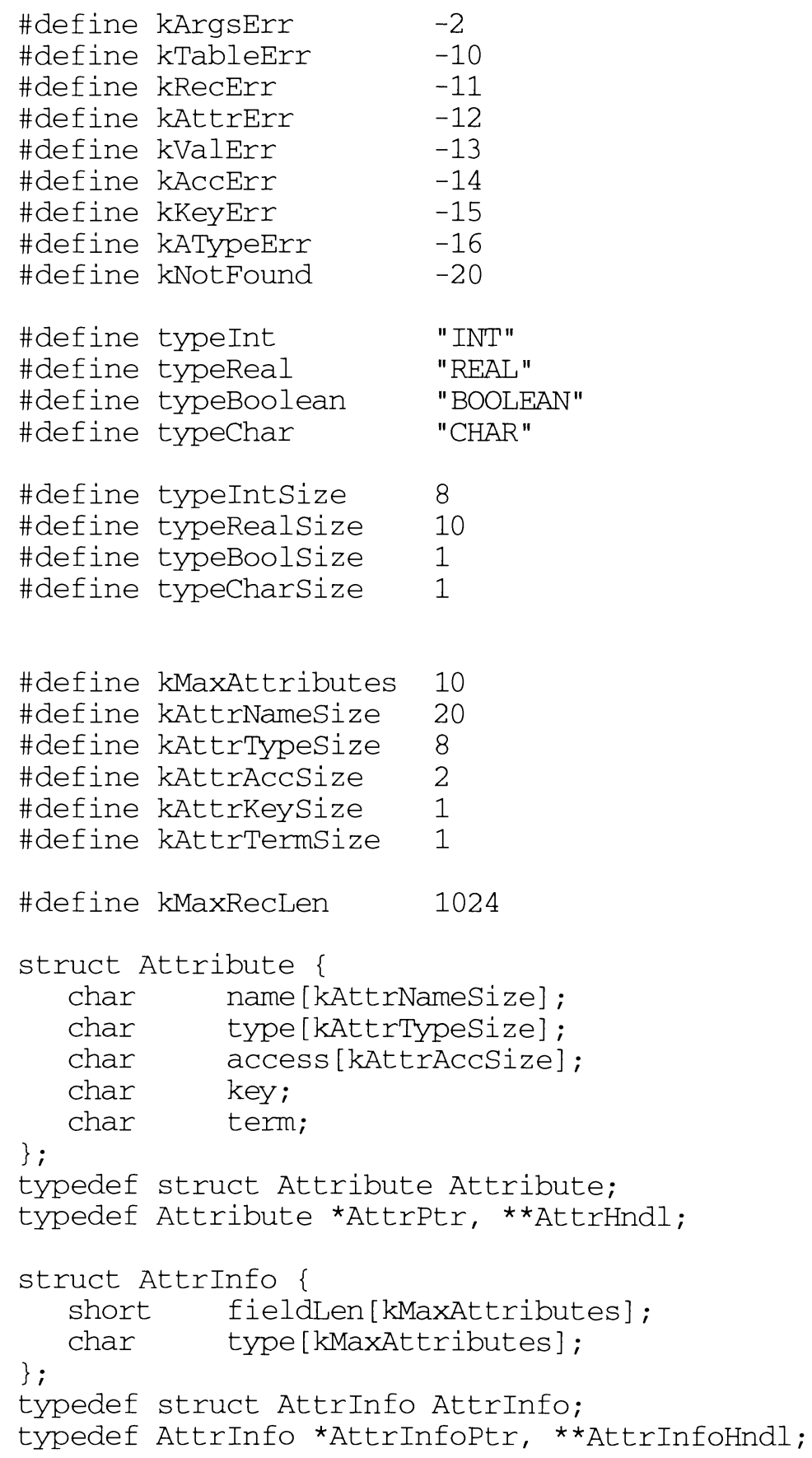


short SearchStringStart (char *in, char *search);

short SearchStringLF (char * str);

Boolean Compstring (char *strA, char *strB);

char * ScanToTab (char * str);

char * ScanToX (char * str, char scan);

short ZeroToNum(char * str);

void IntToZero(short num, char * str);

void Pascal2C(Str255 passtr, char ${ }^{*}$ CStr);

short GetTableInfo (short refNum, short *attrNum, short *recLen);

short GetDetailTableInfo(short refNum, short *attrNum, short *recLen, AttrInfo *attrInfo);

short GetAttroffset (short refNum, char *attrName, char *attrType);

char *AppendValue (char *record, char *str, char *attrType);

short CheckAttrParameter (char *type, char *access, char *key);

IX HyperCard XCMD: XCMD Utilities

History:

1.0

$7 / 26 / 95$

Initial version

Components:

XCMDUtils.C Various Utilty Functions

Macos Lib

Macintosh Toolbox Library

HyperXLib

HyperCard Extension Library

Author:

Pascal Dreer

Part of MS Thesis

Language:

Metrowerks Codewarrior $\mathrm{C}++$ 1.2.2 (Macintosh)

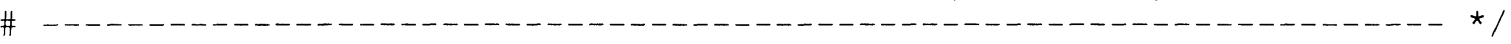

/*

/* Includes * /

/* - .

\#include "XCMDUtils.h"

void EmptyString (char *in, short num)

\{

short i;

for $(i=0 ; i<$ num; $i++)$

\} in $[i]=' \backslash 0^{\prime} ;$ 


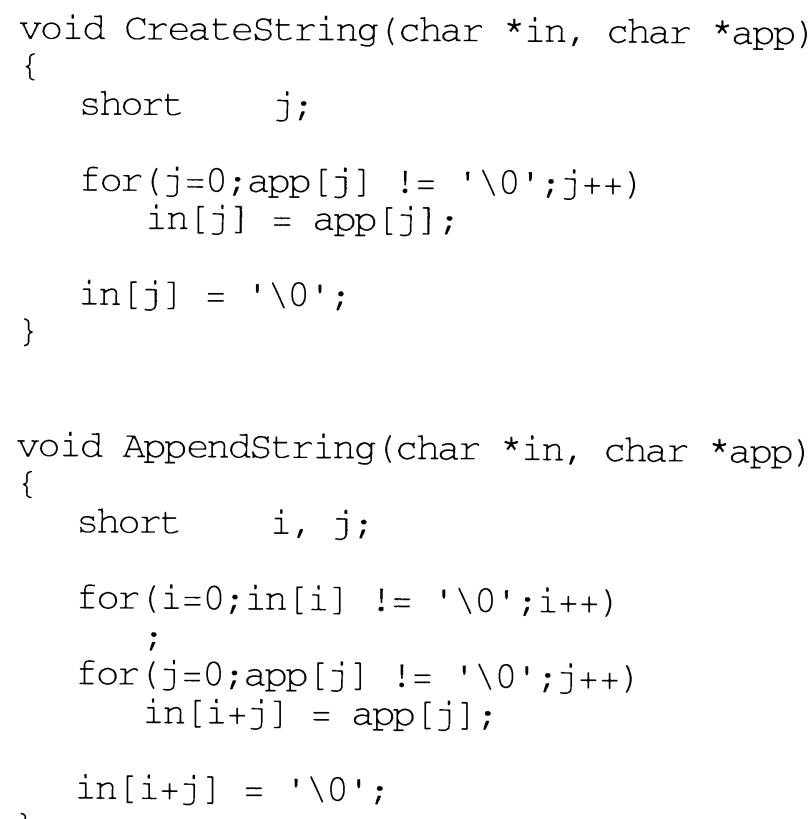




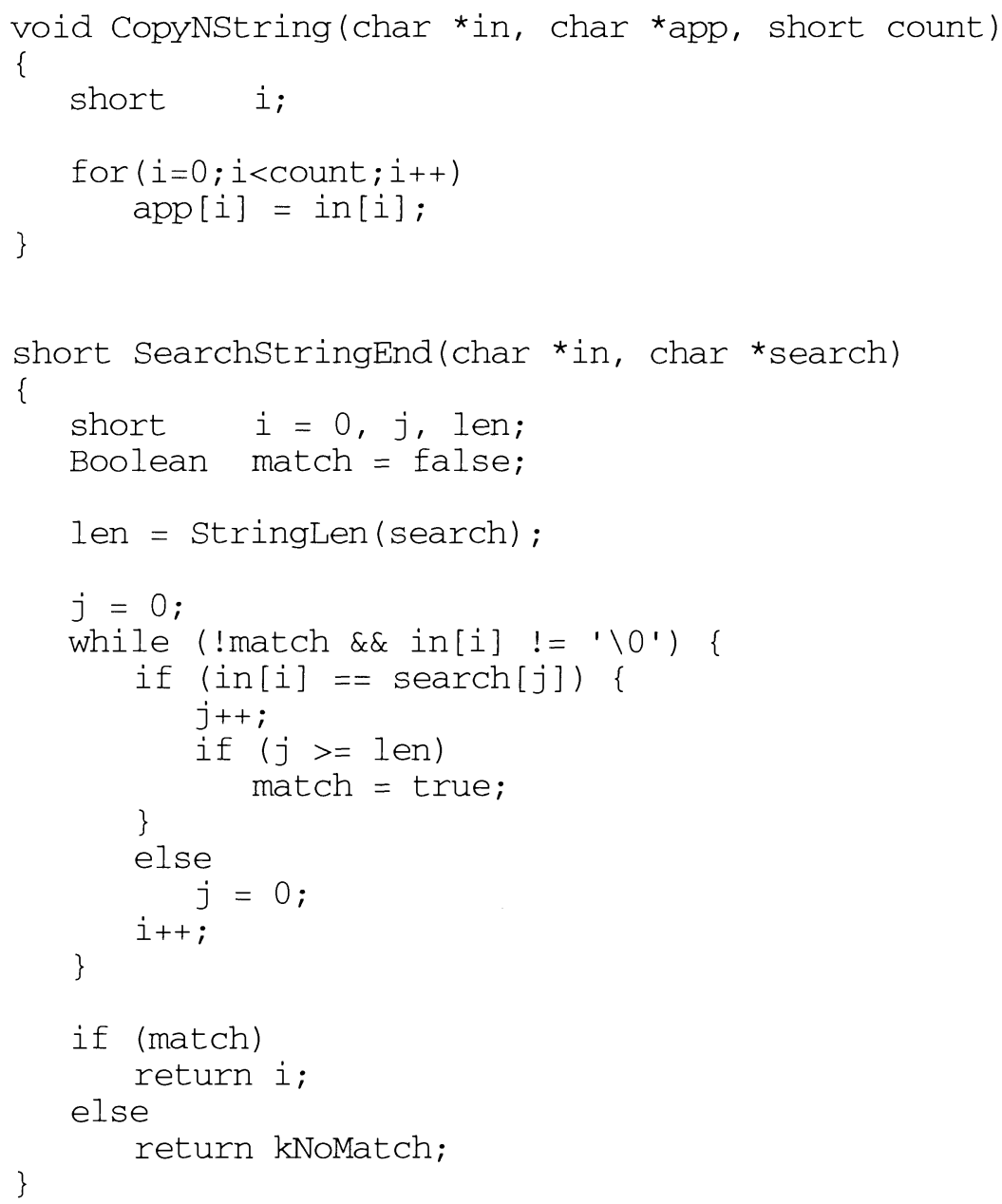




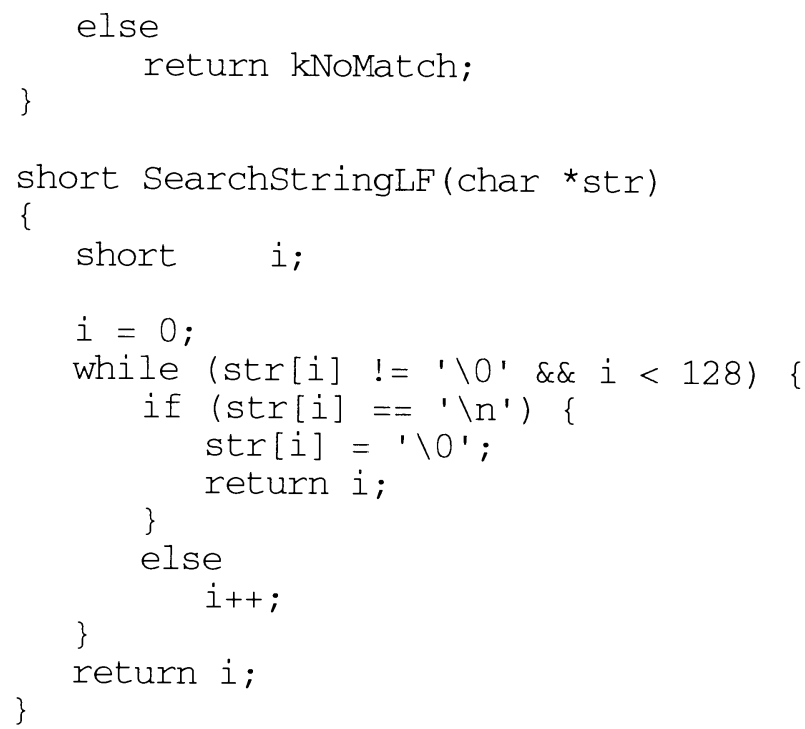




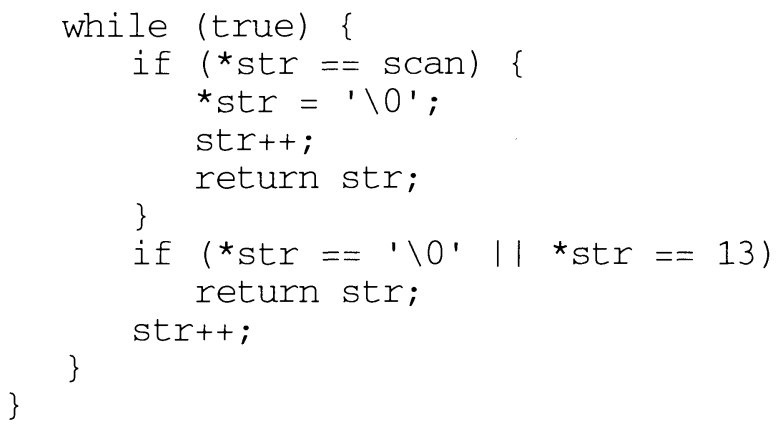




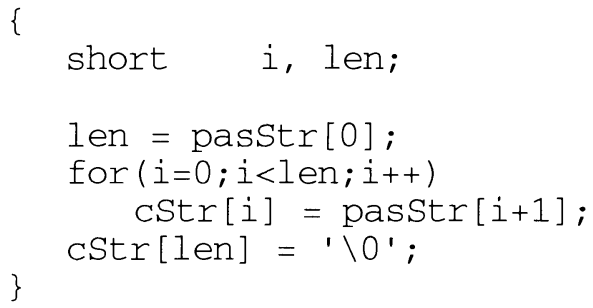


short GetDetailTableInfo(short refNum, short *attrNum, short *recLen, AttrInfo *attrInfo)

\{

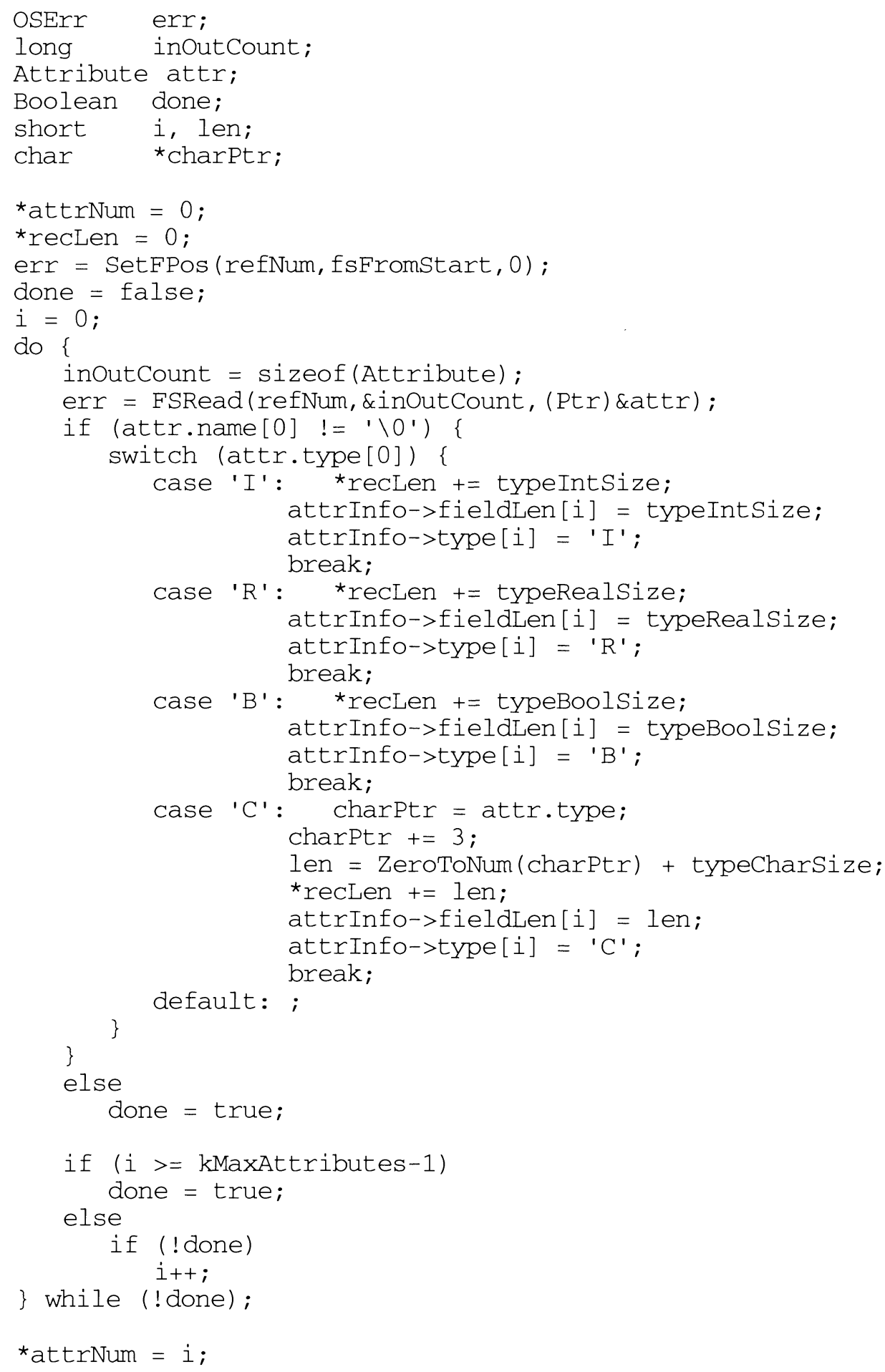


return noErr;

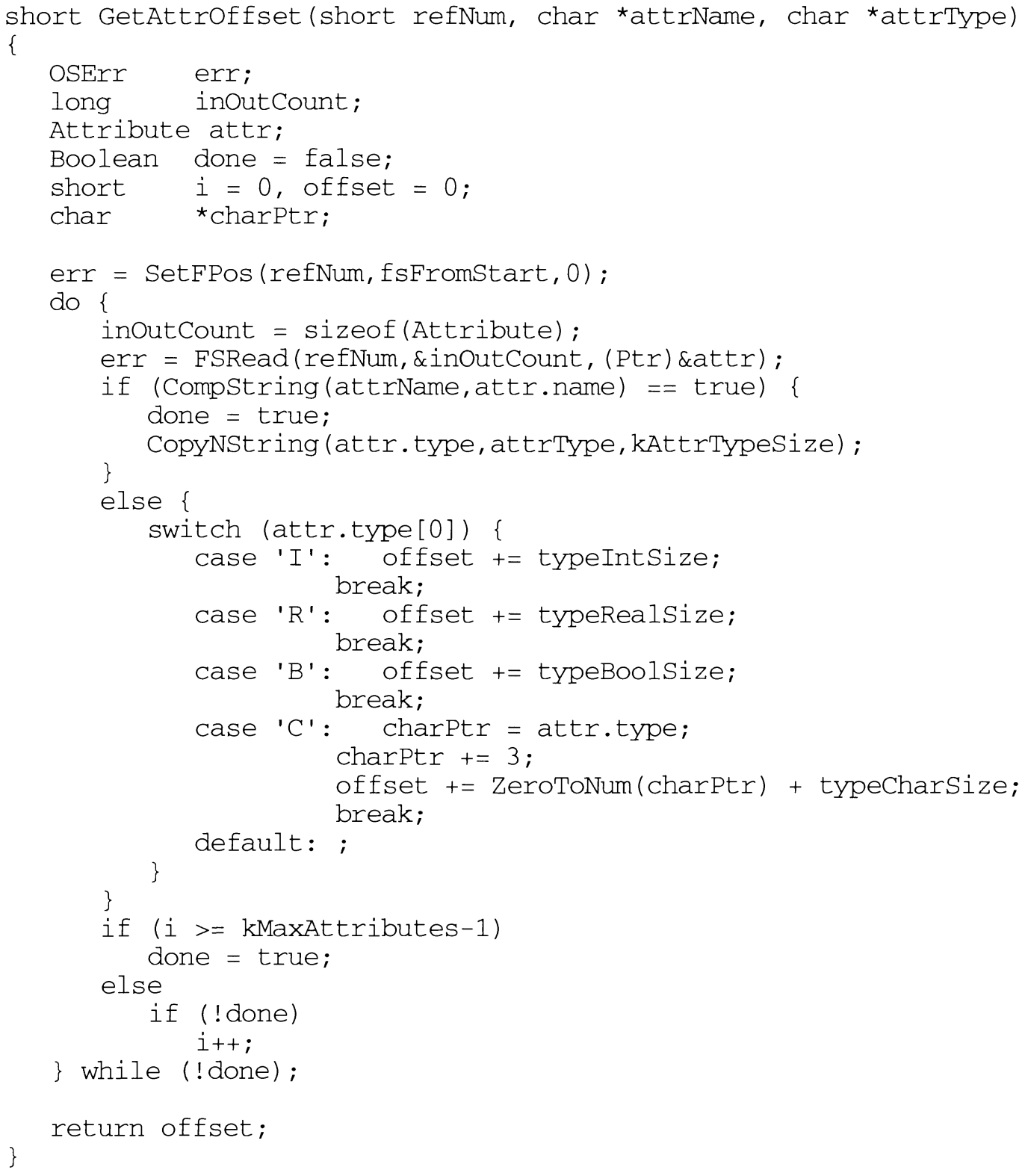




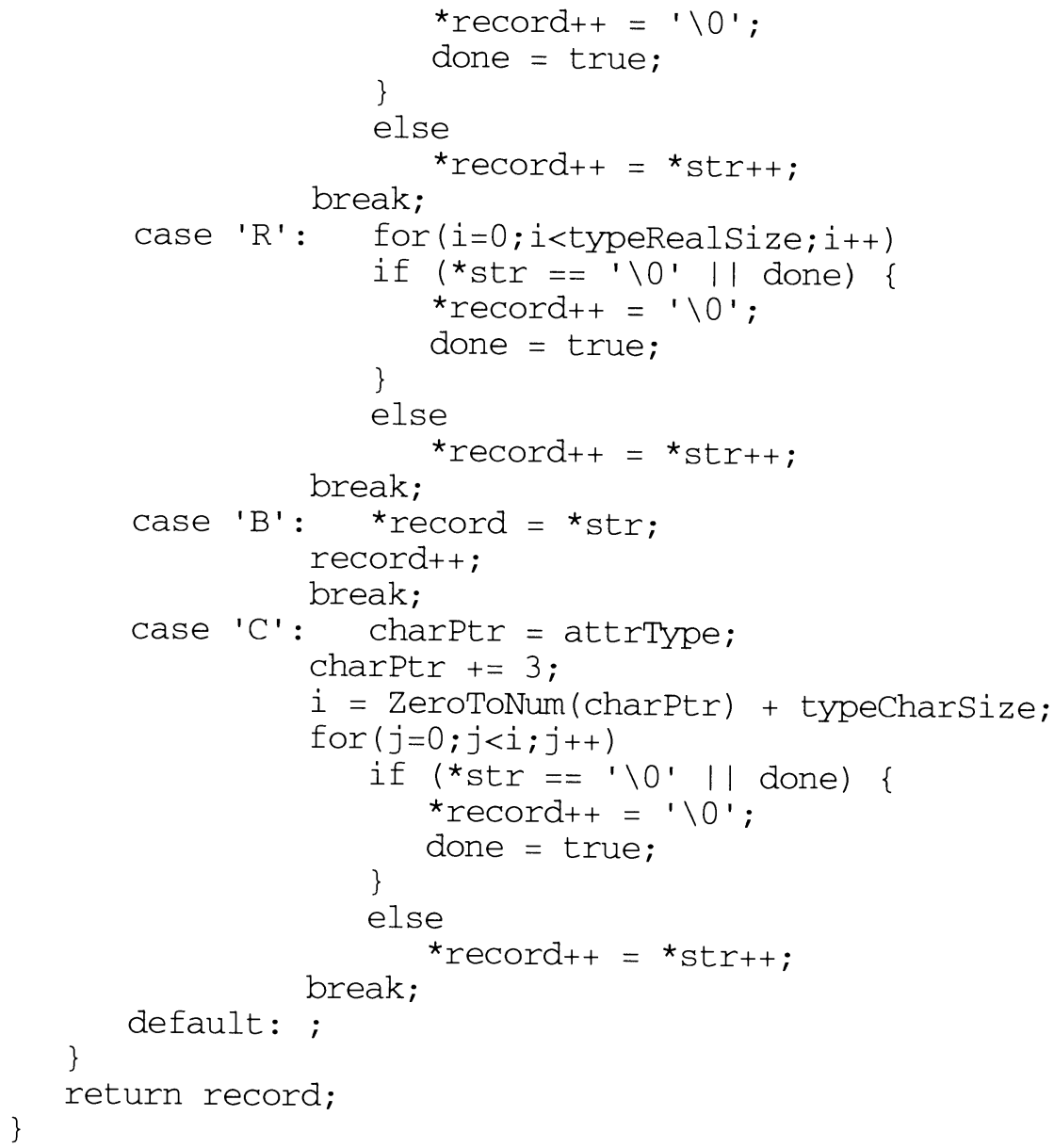

